Louisiana State University

LSU Digital Commons

\title{
Aperiodic Multilayer Graphene Based Tunable and Switchable Thermal Emitter at Mid-infrared Frequencies
}

\author{
Safura Sharifi \\ Louisiana State University and Agricultural and Mechanical College
}

Follow this and additional works at: https://digitalcommons.Isu.edu/gradschool_theses

Part of the Electrical and Computer Engineering Commons

\section{Recommended Citation}

Sharifi, Safura, "Aperiodic Multilayer Graphene Based Tunable and Switchable Thermal Emitter at Midinfrared Frequencies" (2017). LSU Master's Theses. 4416.

https://digitalcommons.Isu.edu/gradschool_theses/4416

This Thesis is brought to you for free and open access by the Graduate School at LSU Digital Commons. It has been accepted for inclusion in LSU Master's Theses by an authorized graduate school editor of LSU Digital Commons. For more information, please contact gradetd@lsu.edu. 


\title{
APERIODIC MULTILAYER GRAPHENE BASED TUNABLE AND SWITCHABLE THERMAL EMITTER AT MID-INFRARED FREQUENCIES
}

\author{
A Thesis \\ Submitted to the Graduate Faculty of the \\ Louisiana State University and \\ Agricultural and Mechanical College \\ in partial fulfillment of the \\ requirements for the degree of \\ Master of Science
}

in

The School of Electrical Engineering and Computer Science

by

Safura Sharifi

B.Sc., Tehran Azad University, 2007

August 2017 


\section{Dedicated}

to my lovely husband,

\section{Yaser;}

from all of life's troubles, you have given me bail. My life has become, a lovely fairytale.

$\&$

to my wonderful daughter,

\section{Persia;}

for being relief and awesome. 


\section{ACKNOWLEDGEMENTS}

Firstly, I would like to express my sincere gratitude to my advisors Dr. Georgios Veronis and Dr. Jonathan P. Dowling for the continuous support of my thesis. I would like to thank Dr. Georgios Veronis for his comments, patience, motivation, and immense knowledge. His guidance and attention to detail helped me in all the time of research and this thesis could not have been written without his supervision. I am truly indebted to Jon for his meticulous comments and encouragements, as well as his innovative approach to develop and widen my research from various perspectives. Not only a great mentor, he has been a true father whose kindness will forever be of immense value to me. I could not have imagined having better advisors and mentors for my M.Sc. study than Dr. Georgios Veronis and Dr. Jonathan P. Dowling.

My sincere thanks also go to Dr. Theda Daniels-Race for her insightful comments and suggestions to improve the quality of my research. Last but not the least, I would like to thank my lovely husband for supporting me spiritually throughout my study and my life in general. Without a doubt, this accomplishment would have never been possible without his continuous support, unconditional love, and prayers. 


\section{TABLE OF CONTENTS}

ACKNOWLEDGEMENTS iii

LIST OF FIGURES vi

LIST OF ABBREVIATIONS xii

LIST OF SYMBOLS xiii

ABSTRACT xvii

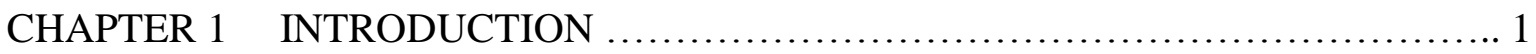

1.1 Historical background and motivation .................................. 1

1.2 Outline of the thesis .............................................. 4

CHAPTER 2 GRAPHENE PROPERTIES AND APPLICATIONS....................... 6

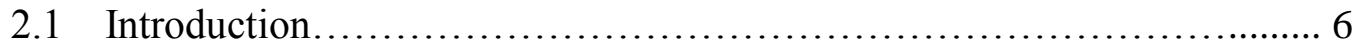

2.2 Graphene and its physical geometry/morphology ....................... 6

2.3 Electronic properties.............................................. 10

2.4 Optical properties............................................... 13

2.5 Graphene photonics applications...................................... 14

CHAPTER 3 PHYSICAL MODELS AND SIMULATION METHOD .................... 20

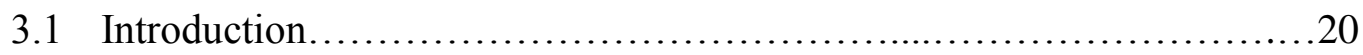

3.2 Thermal emitter / Blackbody radiation ..................................20

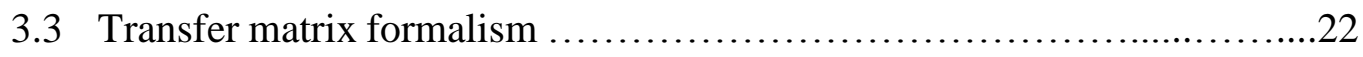

3.4 Genetic algorithm..................................................... 29

3.5 Optical conductivity model of graphene ............................... 30

CHAPTER 4 APERIODIC GRAPHENE BASED MULTILAYER STRUCTURE AS A SELECTIVE, TUNABLE, AND SWITCHABLE THERMAL EMITTER .................. 37

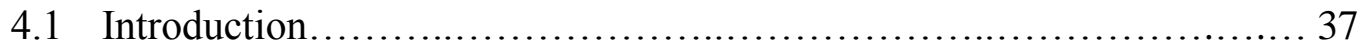

4.2 Structure of graphene-based thermal emitter ............................. 37

4.3 Optimized structure of narrowband thermal emitter ...................... 39

4.4 Tunability and switchability of graphene-based thermal emitter ......... 44 
CHAPTER 5 COMPARISON OF APERIODIC GRAPHENE BASED MULTILAYER THERMAL EMITTERS WITH DIFFERENT NUMBER OF GRAPHENE LAYERS.......47

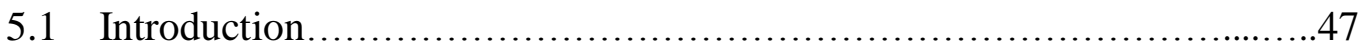

5.2 Optimized graphene based thermal emitters.................................. 47

5.3 Effect of chemical potential on selectivity, tunability, and switchability... 49

CHAPTER 6 CONCLUDING REMARKS AND RECOMMENDED FUTURE

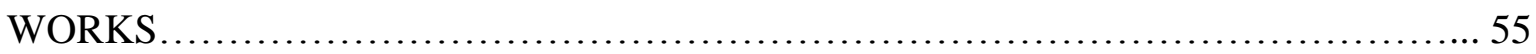

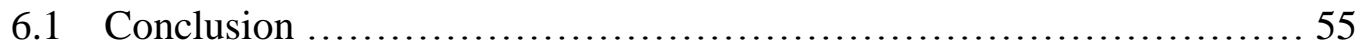

6.2 Recommendation for future works................................... 56

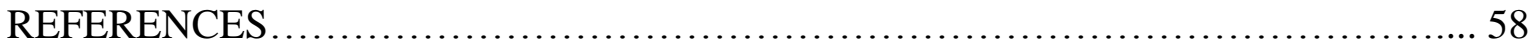

APPENDIX A. DATA FOR THE THICKNESSES OF OPTIMIZED STRUCTURES .. 70

APPENDIX B. LETTERS OF PERMISSION FOR ADOPTED FIGURES ................72

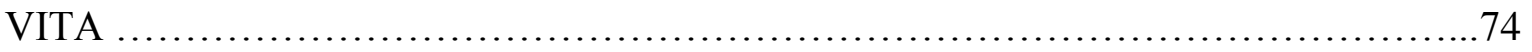




\section{LIST OF FIGURES}

Figure 2.1: Graphene is a 2D building material for carbon materials of all other carbon allotropes which can be wrapped up into 0D fullerenes, rolled into 1D CNTs or stacked into 3D graphite. The figure is adopted by permission from [1]. The permission letter is attached in Appendix B.............................

Figure 2.2: (a) A diagram of the electromagnetic spectrum represented by a rainbow arrow. Applications utilizing different spectral ranges are presented in the top portion of the panel. NIR, MIR, and FIR correspond to near-, mid-, and farinfrared, respectively. The atomic structures of hexagonal boron nitride $(\mathrm{hBN})$, molybdenum disulfide $\left(\mathrm{MoS}_{2}\right)$, black phosphorus (BP), and graphene are shown in the bottom of the panel (from left to right). (b), (c), (d), (e) The bandstructures of a single layer of $\mathrm{hBN}, \mathrm{MoS}_{2}, \mathrm{BP}$, and graphene, respectively. (f) Schematic of a light emitting diode using 2D heterostructures. A narrow gap BP thin film is sandwiched between large gap TMDCs with $\mathrm{p}$ - and n-doping for injection of holes and electrons, respectively. The figure is adopted by permission from [2]. The permission

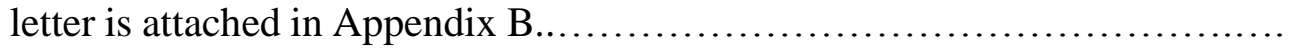

Figure 2.3: (a) Atomic-resolution image of graphene sheet showing individual carbon atoms in white. The figure is adopted by permission from [3]; (b) 2D hexagonal lattice of graphene in real space; (c) First Brillouin zone in momentum space. The figures are adopted by permission from [4]. The permission letter is attached in Appendix B..................................

Figure 2.4: (a) Full dispersion from tight binding calculation and zoom on the low energy region at one of the $\mathrm{K}$ points, the so called Dirac points, adopted by permission from [4]; (b) Conductivity of graphene as a function of carrier density at room temperature. The black line of the experimental data is matched with red dots, obtained by a Boltzmann model of conductivity [5]. The cone-like dispersion as well as the positions of Fermi level (arrow) corresponding to different regions of operation are also shown. The permission letter is attached in Appendix B...............................

Figure 2.5: (a) Photograph of a 50-mm aperture partially covered by graphene and its bilayer. The line scan profile shows the intensity of transmitted white light along the yellow line. The inset is a $20-\mu \mathrm{m}$-thick metal support structure with apertures of 20,30, and $50 \mu \mathrm{m}$ in diameter with graphene placed over them. (b) Transmittance spectrum of single layer graphene (open circles). Slightly lower transmittance for $\lambda<500 \mathrm{~nm}$ is probably due to hydrocarbon contamination. The red line is the transmittance $T=(1+0.5 \pi \alpha)^{-2}$ expected for two-dimensional Dirac fermions, whereas the green curve takes into account the nonlinearity and triangular warping of graphene's electronic spectrum. The gray area indicates the standard error for measurements. The inset shows the transmittance of white light as a function of the number of graphene layers (squares). The dashed lines correspond to an intensity reduction by $\pi \alpha$ with 
each added layer. The figure is adopted by permission from [6]. Permission letter is attached in Appendix B..............................................

Figure 2.6: Graphene photonic applications in display technology (green), electronic devices (blue), as well as optical devices (pink), and interconnects (brown). Application timelines are based on projections of products requiring advanced materials such as graphene, which can be enabled by continuous advances in graphene technology. The figure gives an indication of when a functional device prototype could be expected based on device roadmaps and the development schedules of industry leaders [7]. The quality of graphene and the required synthesis methods are shown for each category of application. The details are beyond the scope of this thesis. The figure is adopted by permission from [7]. The permission letter is attached in Appendix B

Figure 3.1: Electromagnetic spectrum as well as the thermal radiation spectrum including the blackbody radiation at near infrared and mid-infrared wavelengths. The figure is reproduced from another figure in the public domain.

Figure 3.2: $\quad$ A multilayer structure having $m$ layers between a semi-infinite air region and a semi-infinite substrate. Each layer has a thickness of $a_{j}$ and its optical properties are described by its complex index of refraction. The optical electric field at any point in layer $j$ is represented by two components: one propagating in the positive and one in the negative $x$ direction, $E_{j}^{+}$and $E_{j}^{-}$, respectively....

Figure 3.3: $\quad$ Sketch of graphene's dispersion for different chemical potentials. (a) For undoped graphene, all the interband transitions are allowed; (b) For doped graphene, interband transitions are forbidden for $\hbar \omega<2 E_{F}$ by the Pauli blocking; (c) For visible light, the optical conductivity due to interband transitions is dominant; (d) For infrared radiation, the interband and intraband transitions have comparable contributions to the optical conductivity of graphene; (e) The real part and (f) imaginary parts of the refractive index obtained by the Kubo formalism, as well as the equivalent changes in their values for visible and infrared radiation for chemical potential of $\mu_{c}=0.0 \mathrm{eV}$ and $\mu_{c}=0.6 \mathrm{eV}$

Figure 4.1: (a) Structure of the proposed thermal emitter. (b) Lattice structure of graphene and hBN buffer layer.

Figure 4.2: (a) Optimized structure with 23layers of graphene; (b) Normalized power radiated per unit area and unit wavelength by the structure in the normal directon. Note: the layer thicknesses for the structure in (a) are given in appendix $\mathrm{A}$ 
Figure 4.3: (a) Optimized structure with 23 layers of graphene including the location of thinnest hBN layer. (b) Effect of changing the optimized thickness of the thinnest hBN layer on the emittance spectra, as well as on the contribution of graphene layers and tungsten to the total emittance.......................

Figure 4.4: (a) Electric field amplitude normalized with respect to the field amplitude of the incident plane wave for the optimized structure with 23 graphene layers; (b) Contribution of each graphene layer to the total power emitted for $\mu_{c}=$ $0.0 \mathrm{eV}, 0.4 \mathrm{eV}$ and $1.0 \mathrm{eV}$....

Figure 4.5: Effect of changing the chemical potential in graphene, i.e. $\mu_{c}=0.0 \mathrm{eV}, 0.4$ $\mathrm{eV}$, and $1.0 \mathrm{eV}$, on the partial absorptance and the reflectance.

Figure 4.6: Absorptance of the optimized structure in Fig. 4.2(a) for different chemical potentials, $\mu_{c}=0.0 \mathrm{eV}, 0.2 \mathrm{eV}, 0.4 \mathrm{eV}, 0.6 \mathrm{eV}, 0.8 \mathrm{eV}$, and $1.0 \mathrm{eV}$.

Figure 5.1: Aperiodic multilayer structures with 8, 13, 23, 28, and 32 layers of graphene optimized using the genetic algorithm. Note: the layer thicknesses for each structure are given in appendix A.......................................

Figure 5.2: $\quad$ Normalized power radiated per unit area and unit wavelength in the normal direction by the structures of Fig. 5.1 as a function of wavelength............

Figure 5.3: (a) Normalized power emitted per unit area and unit wavelength in the normal direction from bulk tungsten at $\mathrm{T}=873 \mathrm{~K}$. Normalized power emitted, per unit area and unit wavelength in the normal direction as a function of wavelength and chemical potential at $\mathrm{T}=873 \mathrm{~K}$ for the five optimized structures with (b) 8, (c) 13, (d) 23, (e) 28, and (f) 32 layers of graphene. Note: the dotted vertical line shows the wavelength of $\lambda=3.34 \mu \mathrm{m}$ at which the structures are optimized and the dash-dotted lines correspond to $0.7 \times \max$ [ $\bar{\mu}(\lambda)]$, which is used to define the bandwidth of the emission...............

Figure 5.4: Bandwidth $\Delta \lambda$, i.e. selectivity, of the thermal power emitted from the optimized structures with different numbers of graphene layers versus chemical potential. The bandwidth is measured at the wavelengths at which the normalized power emitted becomes $0.7 \times \max [\bar{\mu}(\lambda)]$

Figure 5.5: Tunability of the peak normalized power emitted per unit area and unit wavelength in the normal direction for the optimized structures with different number of graphene layers when the chemical potential is varied..............

Figure 5.6: Switchability of the emitted thermal power from the optimized structures with different number of graphene layers as the chemical potential is varied.............................................................. 


\section{LIST OF ABBREVIATIONS}

\begin{tabular}{|c|c|}
\hline $\mathrm{hBN}$ & hexagonal Boron Nitride \\
\hline $\mathrm{THz}$ & Terra Hertz \\
\hline MIR & Mid-infrared \\
\hline $\mathrm{SiC}$ & Silicon Carbide \\
\hline $\mathrm{BP}$ & Black Phosphorous \\
\hline TMDC & Transition Metal Dichalcogenide Monolayers \\
\hline $\mathrm{CNP}$ & Charge Neutrality Point \\
\hline STM & Scanning Tunneling Microscopy \\
\hline NIR & Near-infrared \\
\hline FLG & Few Layers of Graphene \\
\hline ITO & Indium Tin Oxide \\
\hline FDFD & Finite Difference Frequency Domain \\
\hline FDTD & Finite Difference Time Domain \\
\hline $\mathrm{TM}$ & Transverse Magnetic \\
\hline TE & Transverse Electric \\
\hline $3 \mathrm{D}$ & Three Dimensional \\
\hline $2 \mathrm{D}$ & Two Dimensional \\
\hline $1 \mathrm{D}$ & One Dimensional \\
\hline OD & Zero Dimensional \\
\hline LED & Light Emitting Diode \\
\hline OLED & Organic Light Emitting Diode \\
\hline LCD & Liquid Crystal Display \\
\hline
\end{tabular}




\section{LIST OF SYMBOLS}

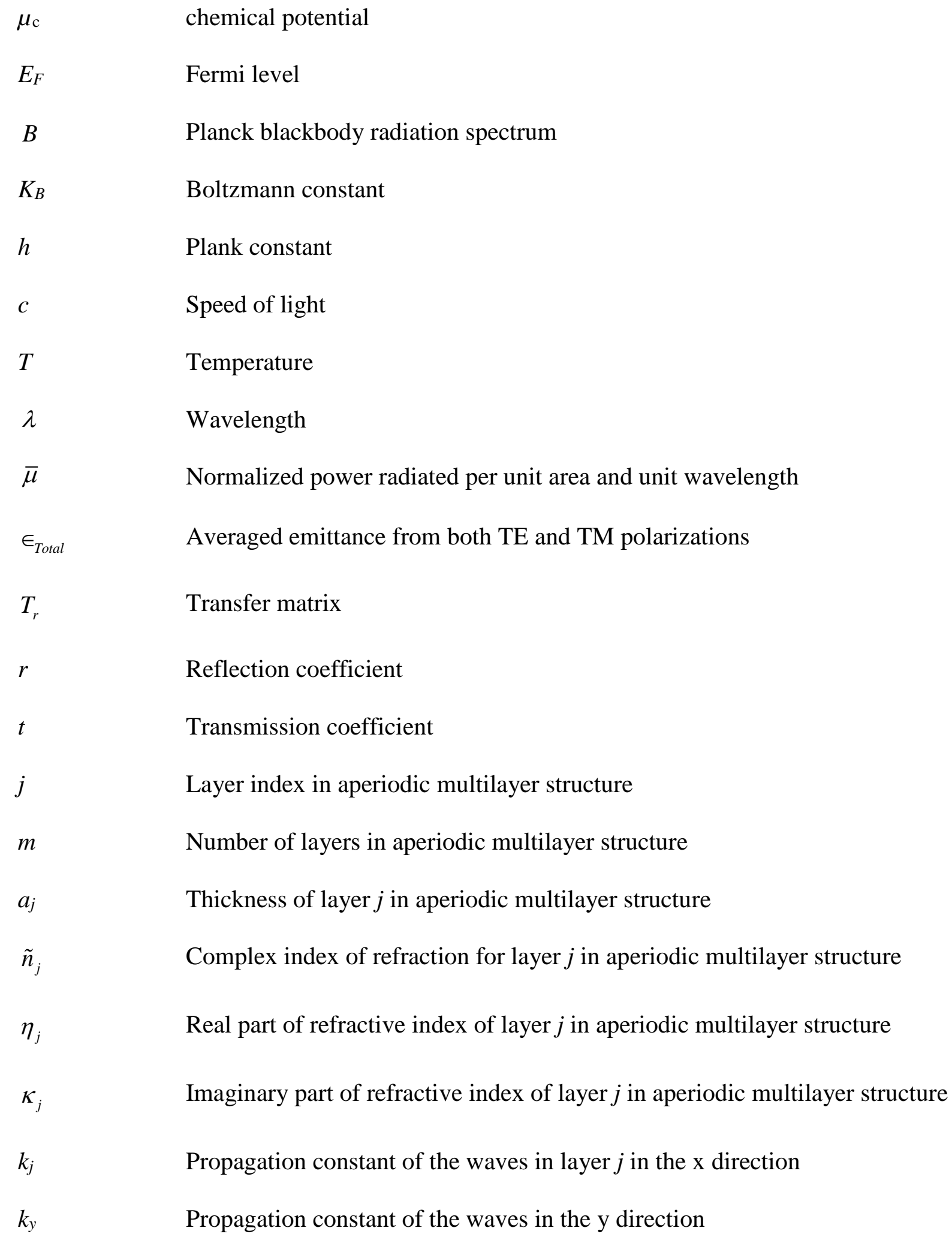




\begin{tabular}{|c|c|}
\hline$Z_{j}$ & Free-space impedance \\
\hline$\mu_{0}$ & Free-space magnetic permeability \\
\hline$\varepsilon_{0}$ & Free-space dielectric permittivity \\
\hline$\varepsilon_{j}$ & Dielectric permittivity of the material in layer $j$ \\
\hline$E_{z}$ & Transverse component of the electric field for TM polarization \\
\hline$H_{y}$ & Transverse component of the magnetic field for TM polarization \\
\hline$E_{j}^{+}(x)$ & Electric field component propagating in layer $j$ in the positive $x$ direction \\
\hline$E_{j}^{-}(x)$ & Electric field component propagating in layer $j$ in the negative $x$ direction \\
\hline$I_{j, j-1}$ & Interface matrix between layers $j$ and $j-1$ in aperiodic multilayer structure \\
\hline$r_{j, j-1}$ & Fresnel complex reflection coefficient at interface between layers $j$ and $j-1$ \\
\hline$t_{j, j-1}$ & Fresnel complex transmission coefficient at interface between layers $j$ and $j-1$ \\
\hline$\eta_{0}$ & Refractive index of air \\
\hline$\phi_{0}$ & Angle of incidence \\
\hline$\phi_{j}$ & Angle of refraction in layer $j$ \\
\hline$L_{j}$ & Phase matrix describing the propagation through layer $j$ \\
\hline$S$ & Total system transfer matrix \\
\hline$S_{j}^{\prime}$ & Left subset of internal electric field in layer $j$ \\
\hline$S^{\prime \prime}$ & Right subset of internal electric field in layer $j$ \\
\hline$t_{j}^{+}$ & Internal electric field propagating in the positive $\mathrm{x}$ direction in layer $j$ \\
\hline$t_{j}^{-}$ & Internal electric field propagating in the negative $\mathrm{x}$ direction in layer $j$ \\
\hline
\end{tabular}




$\begin{array}{ll}E_{j} & \text { Total electric field in an arbitrary plane in layer } j \\ E_{0}^{+} & \text {Electric field of incident plane wave } \\ Q_{j}(x) & \text { Time average of the energy dissipated per unit time in layer } j \text { at position } x \\ \leftrightarrow & \text { Magneto-optical surface complex conductivity tensor } \\ \omega & \text { Radian frequency } \\ \Gamma & \text { Charged particle scattering rate } \\ \mathbf{E}_{\mathbf{0}} & \text { DC electric field } \\ \mathbf{B}_{0} & \text { DC magnetic field } \\ \overrightarrow{\mathbf{I}}_{\mathrm{t}} & \text { Symmetric dyads } \\ \overrightarrow{\mathbf{J}}_{\mathrm{t}} & \text { Antisymmetric dyads } \\ \sigma_{d} & \text { Diagonal Hall conductivity } \\ \sigma_{0} & \text { Off-diagonal Hall conductivity } \\ \sigma_{F} & \text { Fermi-Dirac distribution } \\ \sigma_{d, \text {,inter }} & \text { Extra }\end{array}$


$\tau$

$\varepsilon_{G}$

$t_{G}$

K

$\mathrm{eV}$
Relaxation time

Dielectric permittivity of graphene monolayer

Thickness of a single graphene layer

Kelvin (Absolute temperature unit)

Electron Volt 


\begin{abstract}
Over the past few decades, there have been tremendous innovations in electronics and photonics. The development of these ultra-fast growing technologies mostly relies on fundamental understanding of novel materials with unique properties as well as new designs of device architectures with more diverse and better functionalities. In this regard, the promising approach for next-generation nanoscale electronics and photonics is to exploit the extraordinary characteristics of novel nanomaterials.

There has been an explosion of interest in graphene for photonic applications as it provides a degree of freedom to manipulate electromagnetic waves. In this thesis, to tailor the broadband blackbody radiation, new aperiodic multilayer structures composed of multiple layers of graphene and hexagonal boron nitride $(\mathrm{hBN})$ are proposed as selective, tunable and switchable thermal emitters. To obtain the layer thicknesses of these aperiodic multilayer structures for maximum emittance/absorptance, a hybrid optimization algorithm coupled to a transfer matrix code is employed.

The device simulation indicates that perfect absorption efficiency of unity can be achieved at very narrow frequency bands in the infrared under normal incidence. It has been shown that the chemical potential in graphene enables a promising way to design electrically controllable absorption/emission, resulting in selective, tunable and switchable thermal emitters at infrared frequencies. By simulating different aperiodic thermal emitters with different numbers of graphene layers, the effect of the number of graphene layers on selectivity, tunability, and switchability of thermal emittance is investigated. This study may contribute towards the realization of wavelength selective detectors with switchable intensity for sensing applications.
\end{abstract}




\section{CHAPTER 1 \\ INTRODUCTION}

\subsection{Historical background and motivation}

At finite temperatures, all materials emit electromagnetic radiation due to the thermally induced motion of particles and quasiparticles [8]. A perfect thermal emitter follows Planck's law of blackbody radiation, which is broadband, incoherent and isotropic, with a spectral profile and intensity that are dependent on the emissivity of a material, and that vary only with changes in temperature. However, it is desirable to have an emitter that radiates only within a certain frequency bandwidth, i.e. a selective emitter, for various applications, including infrared sensing, thermal imaging, and thermophotovoltaics. The spectral features of the controlled thermal emission (e.g. wavelength, bandwidth, peak emissivity, and angular characteristics) are strongly dependent on the choice of both materials and structures of the emitters. It has been shown that the directionality and coherence of blackbody emission can be controlled by nanoengineered structures such as patterned gratings [9, 10], photonic crystals [11, 12], microcavity resonators [13-16], metasurfaces $[8,17,18]$, and graphene nanostructures $[19,20]$. Using the appropriate design of optical nanostructures, arbitrary shaping of thermal emission spectra can be realized, from single-peak ultra-narrowband emission for mid-infrared sensing [21] to a stepwise emissivity spectrum for thermophotovoltaics [22]. Coherent infrared thermal emitters with tunable emitting frequencies in a broad spectral range are highly desired for numerous promising applications in energy harvesting [16, 23, 24], chemical sensing [25], infrared (IR) sources [26], thermal circuits $[19,27]$, and radiative cooling [28].

The basic method to achieve narrowband absorptivity (emissivity) is the direct use of rare

earth oxides, which inherently cause strong absorption at fixed wavelengths [29, 30]. However, the arbitrary control of an emission wavelength or an emission bandwidth is limited to the 
properties of chosen materials that determine the magnitude of interaction between light and materials. A selective emitter can be achieved by photonic bandgaps in photonic crystals composed of metallic and dielectric structures [9,31]. As electromagnetic fields are strongly attenuated below the plasma frequency of metals $[32,33]$, they introduce more flexibility in creating a thermal emitter with broadband frequency selectivity $[34,35]$. In addition, metals are potentially suitable for near-infrared selective thermal emitters, since they have large absorption in the near infrared frequencies with stable properties at high temperatures. However, metals have also high reflectivity in mid- and far- infrared frequencies and consequently structures composed of metals can potentially show a low emissivity [36]. As such, the surface is required to be modified periodically by an array of grooves [34] or holes [35] to enhance emission at infrared frequencies. In this way, the radiative and absorptive rate of the photonic crystal resonances dictates the emissivity spectrum so that the geometrical parameters enable a broadband, selective thermal emitter.

A narrowband thermal emission can be achieved using metallic nanostructures so that the optical resonant modes, confined in so-called Salisbury screen [37] and Fabry-Perot cavity [38, 39], are excited on the metal surface, leading to enhanced absorptivity (emissivity) at those resonant wavelengths $[12,18,22,40-43]$. According to the Purcell effect [44], thermal radiation from an optical resonator can be dramatically modulated by the resonance mode designed in the infrared range, leading to narrowband thermal emission at the resonant frequency. Liu et al. [45] demonstrated that the matched mode of the emitter can be lost when the resonance mode is electrically quasi-static, i.e. the electric field oscillates in phase, resulting in the fundamental limit of the spectral thermal emission power from an optical resonator. 
Metamaterials based structures have also led to narrowband thermal emission [8, 46-52]. The effective permittivity and permeability of the entire structure are artificially controlled by combining subwavelength metallic elements with thin dielectric layers in a properly designed structure [53], leading to perfect absorption [9, 44, 54, 55] (maximum emission) at the resonant wavelengths. It should be noted, however, that strong free carrier absorption due to metals leads to undesired emission over an extensive wavelength range together with the broadening of the emission peaks in selective thermal emitters designed by photonic crystals and metamaterials [56]. In addition, a narrowband resonance achieved in these structures cannot be changed dynamically to other operation frequencies due to the limitation in the properties and functionalities of available conventional metals.

Infrared frequency-tunable coherent thermal emitters play an important role in material analysis providing noninvasive information on chemical composition and bonds. The dynamic control of thermal radiation has been demonstrated through in situ modification of material emissivity. This has been achieved with nanophotonic structures that incorporate phase change materials so that the emissivity can be electronically manipulated by controlling the charge injection, and consequently the polariton modes in the structure. Cong et al. [57] demonstrated that a tunable selective absorber can be designed by InSb, whose carrier density can be adjusted by utilizing optical pump or changing the surrounding temperature, altering the resonance frequency of split rings. Similarly, tunable perfect thermal emitters could be designed by the genesis of new materials as well as novel design of photonic and electronic structures.

Graphene, an atomic layer of carbon, provides a unique platform for controlling thermal emittance at infrared wavelengths. It has excellent electronic, photonic, mechanical, and thermal properties. For instance, graphene is a zero-bandgap semi-metal with high carrier mobility at room 
temperature that allows strong interaction with terahertz (THz) and mid-infrared (MIR) waves [58, 59]. The propagation of these waves can be actively controlled by varying the chemical potential ${ }^{1}$ in graphene, which can be tuned by chemical doping, voltage bias, external magnetic field, or optical excitation [7]. However, graphene has low single-pass optical absorption due to its singleatom-layer thickness and thus total absorption can be only achieved by novel designs of graphenebased nanostructures. Thongrattanasiri et al. [20] demonstrated tunable perfect absorbers with graphene ribbon array on dielectric spacer and metallic substrate [20,60]. Wang et al. [61] demonstrated an infrared (IR) frequency-tunable selective thermal emitter made of graphenecovered silicon carbide ( $\mathrm{SiC}$ ) grating whose resonance frequency can be dynamically tuned by $\sim 8.5 \%$ by varying graphene's chemical potential. Fang et al. [62] demonstrated tunable selective absorption in graphene disk arrays.

\subsection{Outline of the thesis}

In this thesis, new graphene-based aperiodic multilayer structures are presented as selective, tunable, and switchable infrared thermal emitters. The remainder of this thesis is organized as follows. In Chapter 2, the structure of graphene and other two-dimensional (2D) materials are first introduced, followed by a summary of graphene's electrical and photonic properties. By conducting a brief literature review, several graphene nanostructures for photonic applications are presented as well.

Chapter 3 is dedicated to physical models, as well as simulation methods and equations. The blackbody radiation of thermal emitters is first presented, followed by the transfer matrix

\footnotetext{
${ }^{1}$ The chemical potential and Fermi level basically correspond to the same physical quantity in material science so that the Fermi level $E_{F}$ is the total chemical potential $\mu_{\mathrm{c}}$ for electrons in the system. As both terms have been frequently used in the literature, these two terms are used interchangeably throughout this thesis.
} 
equations for simulating the absorptance/emittance of aperiodic multilayer structures. Then a hybrid optimization method, consisting of a micro-genetic global optimization algorithm coupled to a local optimization algorithm, is briefly introduced. This method is employed for finding the thicknesses of layers in the proposed aperiodic multilayer structure. Finally, to calculate the optical conductivity of graphene, the Kubo formalism is presented, followed by a discussion of the effect of changing the chemical potential on graphene's refractive index.

In Chapter 4, the proposed graphene-based aperiodic thermal infrared emitter is presented. Using a genetic optimization algorithm and a transfer matrix code, the layer thicknesses and materials are optimized to maximize the absorptance/emittance for normal light incidence at a single wavelength. Then, the simulation results are studied, focusing on the selectivity, tunability, and switchability of this infrared thermal emitter.

Chapter 5 presents five different aperiodic thermal emitters with 8, 13, 23, 28, and 32 layers of graphene. These aperiodic multilayer structures are optimized using genetic optimization algorithm to determine the best structure's dimensions at a given wavelength in mid-infrared range. Using the optimized aperiodic structures, the effect of the number of graphene layers on selectivity, tunability, and switchability of thermal emittance are studied. Finally, in Chapter 6, the conclusions and recommendations for future work are summarized. 


\section{CHAPTER 2 \\ GRAPHENE PROPERITIES AND APPLICATIONS}

\subsection{Introduction}

In this chapter, the structure of graphene and other 2D materials are first presented, followed by describing the possible spectral ranges covered by some of these materials. Then, the electronic and photonic properties of graphene are presented. At the end, the promising applications of graphene are briefly overviewed along with the expected time for their implementation.

\subsection{Graphene and its physical geometry/morphology}

Graphene is a one-atom thick sheet of $\mathrm{sp}^{2}$ hybridized carbon atoms arranged in a honeycomb lattice, and thereby the thinnest possible material in nature. Since the middle of 20th century, graphene has been studied theoretically as the building block of carbon allotropes, as shown in Fig. 2.1 [1]. It can be wrapped into closed zero-dimensional (0D) fullerenes, rolled into one-dimensional (1D) carbon nanotubes (CNTs), or stacked into three-dimensional (3D) graphite. However, graphene as a material on its own was first discovered in 2004 [63], because the stability of $2 \mathrm{D}$ crystals was not considered to be possible for a long time due to thermodynamic fluctuations [64]. The mechanical cleavage of graphite paved the way for isolating a single layer of graphene deposited on an oxidized silicon substrate. This groundbreaking experiment was considered as the first observation of a stable 2D material, which led to the award of the Nobel Prize in Physics in 2010 [65]. Since its discovery, graphene has soon emerged as a promising nanomaterial for novel applications in electronics as well as in photonics due to its remarkable electrical and optical properties, which will be explained to some extent in this chapter.

The discovery of graphene opened up a new field of research for many other 2D materials wherein the thickness of the materials under investigation is orders of magnitude smaller than the 
wavelength of the light involved. The wide range of material properties makes it possible to construct various nanophotonic devices by combining 2D materials with different number of layers and compositions. Many 2D materials strongly interact with light and cover a very wide electromagnetic spectrum from microwave to ultraviolet due to their diverse electronic properties, as shown in Fig. 2.2 [2]. For instance, the gapless nature of graphene attracted significant attention due to its strong interaction with photons in a wide energy range from microwave to ultraviolet $[66,67]$, as well as its high carrier mobility for high speed applications in a broad wavelength range. The absorption coefficient of graphene exceeds $5 \times 10^{7} \mathrm{~m}^{-1}$ in the visible if it is normalized to its atomic thickness, which is more than 10 times larger than those in gallium arsenide and silicon $[2,68]$. As such, the interaction with light can be further enhanced using graphene multilayers for practical device applications.

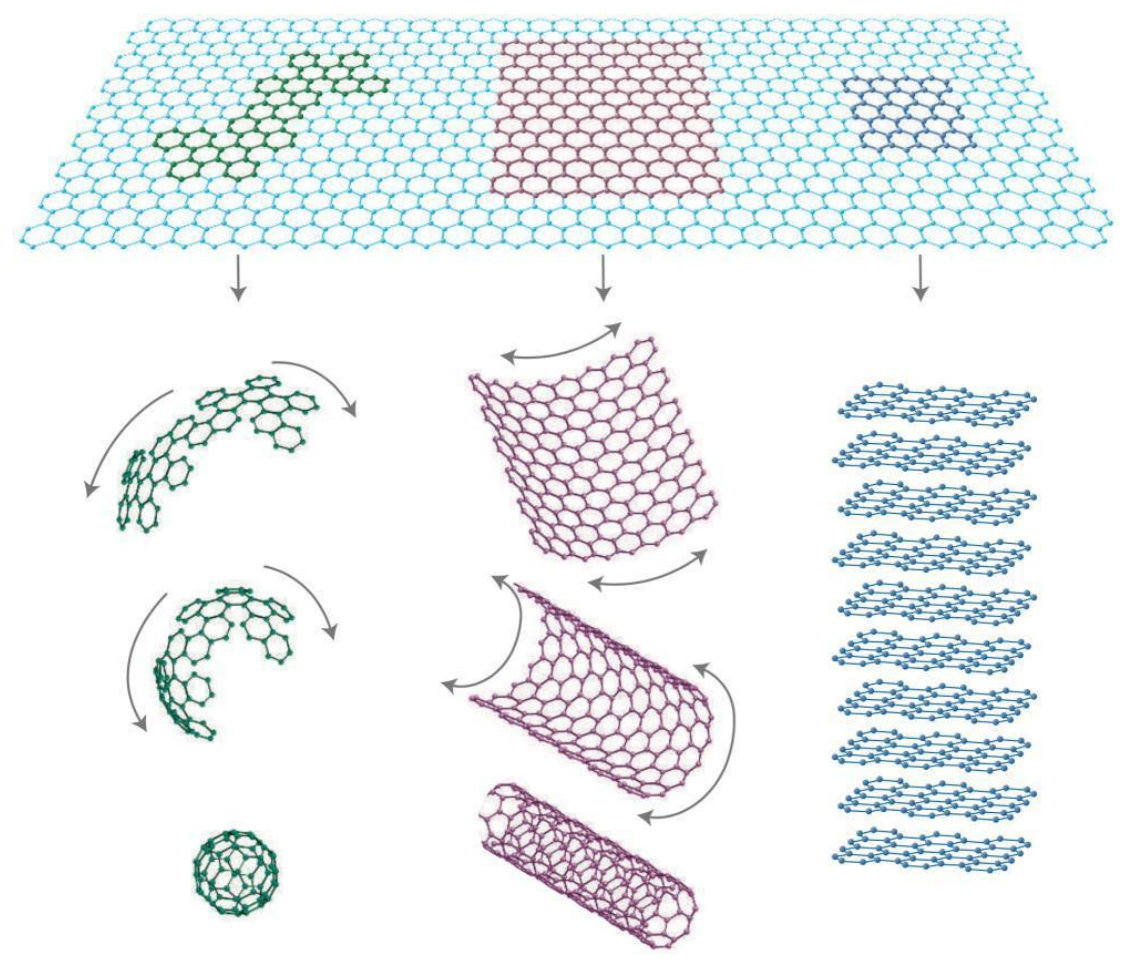

Figure 2.1: Graphene is a 2D building material for carbon materials of all other carbon allotropes which can be wrapped up into 0D fullerenes, rolled into 1D CNTs or stacked into 3D graphite. The figure is adopted by permission from [1]. The permission letter is attached in Appendix B. 
$\mathrm{hBN}$ is another important type of 2D material $[69,70]$ as shown in Fig. 2.2 (b). Since $\mathrm{hBN}$ has $2 \mathrm{D}$ atomic structure similar to graphene, it has been proposed as a promising complementary insulator layer [71]. $\mathrm{hBN}$ can be incorporated in various heterostructures for insulating other conducting 2D materials due to its large bandgap of $\sim 6 \mathrm{eV}$. Single layer transition metal dichalcogenides (TMDC) such as $\mathrm{MoS}_{2}$ and $\mathrm{WSe}_{2}$ are also direct bandgap semiconductors [72, 73], as shown in Fig. 2.2(c). These 2D materials have a sizable optical bandgap greater than $1 \mathrm{eV}$ that leads to many applications such as the realization of efficient light emitting devices. Another recently discovered layered material with a direct bandgap is black phosphorus (BP) [74] [Fig. 2.2(d)]. The bandgap of BP is sizable: from $\sim 0.3 \mathrm{eV}$ in its bulk form to $\sim 2 \mathrm{eV}$ by reducing the number of layers $[75,76]$, allowing the realization of mid-infrared photonic devices in a broad wavelength range.

The photonic devices can be constructed in the form of vertical heterostructures using different 2D materials such as 2D semiconductors $\left(\mathrm{MoS}_{2}, \mathrm{BP}\right), 2 \mathrm{D}$ insulator layers $(\mathrm{hBN})$ and 2D metallic layers (graphene, $\mathrm{TiS}_{2}$, etc.). Figure 2.2(f) shows such an infrared light emitting diode (LED), which consists of both narrow gap BP and larger gap TMDCs. Here, because of its tunable direct bandgap, BP can be utilized as an active material for tunable light emission [76], graphene can be used to minimize contact resistance, and TMDC materials such as p-type $\mathrm{WSe}_{2}$ and n-type $\mathrm{MoS}_{2}$, can be used for injection of holes and electrons into active layers. The injected carriers are trapped within BP for light emission due to the offset of bandgap energy at the BP/TMDC interfaces. Such an ultra-dense hybrid structure is not affected by lattice mismatch because different lattices of 2D materials are only weakly bonded by van der Waals forces without any dangling bonds. 

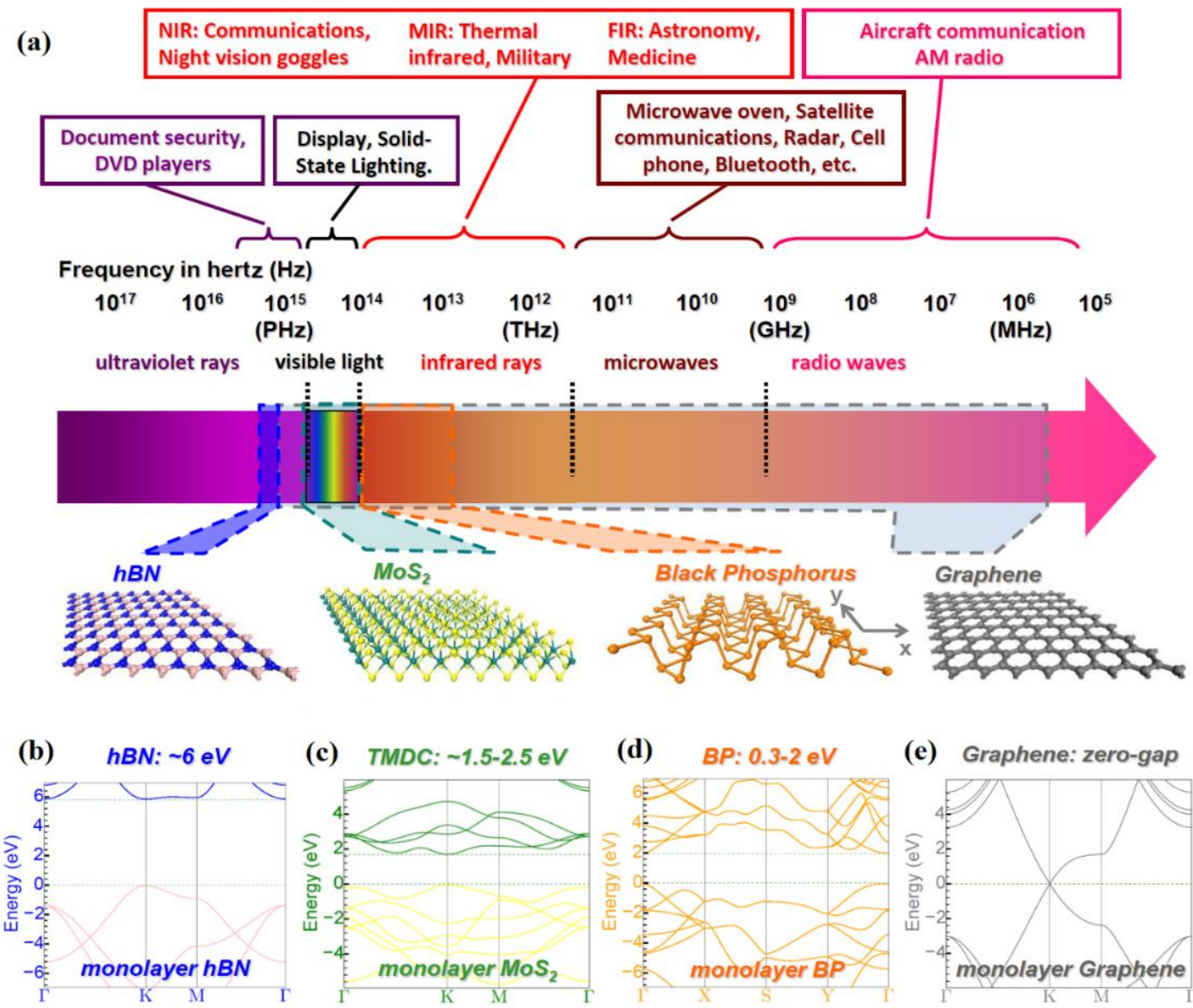

(c) TMDC: $\sim 1.5-2.5 \mathrm{eV}$

(d) $B P: 0.3-2 \mathrm{eV}$

(e) Graphene: zero-gap
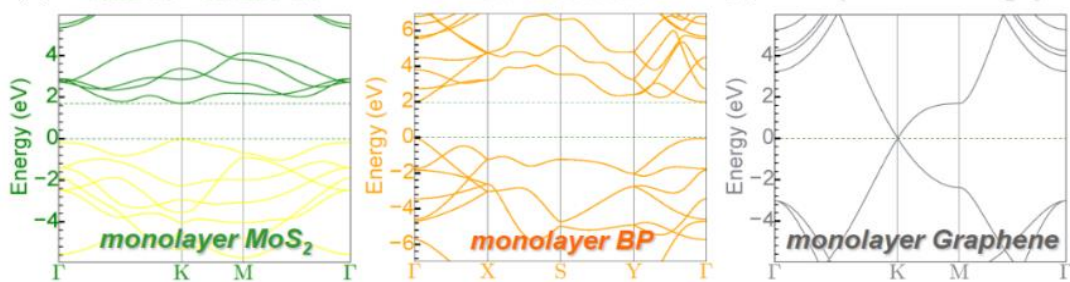

n-type $\mathrm{MoS}_{2}$

Graphene Contact

(f)
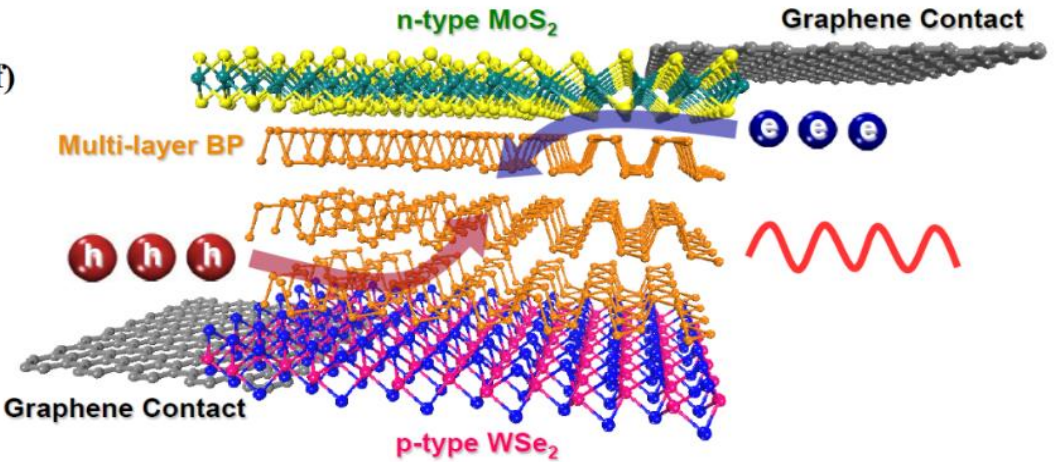

Figure 2.2: (a) A diagram of the electromagnetic spectrum represented by a rainbow arrow. Applications utilizing different spectral ranges are presented in the top portion of the panel. NIR, MIR, and FIR correspond to near-, mid-, and far-infrared, respectively. The atomic structures of hexagonal boron nitride $(\mathrm{hBN})$, molybdenum disulfide $\left(\mathrm{MoS}_{2}\right)$, black phosphorus (BP), and graphene are shown in the bottom of the panel (from left to right). (b), (c), (d), (e) The bandstructures of a single layer of $\mathrm{hBN}, \mathrm{MoS}_{2}$, $\mathrm{BP}$, and graphene, respectively. (f) Schematic of a light emitting diode using 2D heterostructures. A narrow gap BP thin film is sandwiched between large gap TMDCs with p- and n-doping for injection of holes and electrons, respectively. The figure is adopted by permission from [2]. The permission letter is attached in Appendix B. 


\subsection{Electronic properties}

The band structure of graphene can be obtained from nearest-neighbor tight binding calculation on the out-of-plane $2 p_{z}$ orbitals [77]. The hexagonal lattice of graphene, as well as the corresponding Brillouin zone are shown in Fig. 2.3. By assuming the interactions between first nearest neighbor carbon atoms, the closed form of the dispersion relation of graphene can be calculated [4] as follows:

$$
E(\vec{k})= \pm t \sqrt{1+4 \cos \frac{\sqrt{3} k_{x} a_{c c}}{2} \cos \frac{k_{y} a_{c c}}{2}+4 \cos ^{2} \frac{k_{y} a_{c c}}{2}}
$$

where $a_{c c}=1.42 \AA$ is the carbon-carbon atomic distance, $k_{x}$ and $k_{y}$ are wave vectors in $x$ and $y$ directions, and $t=2.8 \mathrm{eV}$ is the nearest neighbor hopping energy. Figure 2.4(a) shows the full band structure of graphene. It can be observed that there are two sets of three cone-like points $K$ and $K^{\prime}$ on the edge of the Brillouin zone, where the conduction and valence bands meet each other in momentum space. The behavior of charge carriers near these points resembles the Dirac spectrum for massless fermions [78], thereby named Dirac points. The dispersion relation near Dirac points can be assumed linear. This cone-like approximation leads to the square root dependence of the charge density on the Fermi level, i.e. the chemical potential, with respect to the value at the Dirac point as follows [4]:

$$
E\left(\overrightarrow{k^{\prime}}\right)= \pm \hbar v_{F}\left|\overrightarrow{k^{\prime}}\right| \Rightarrow E_{F}= \pm \hbar v_{F} \sqrt{\pi n}
$$

where $\hbar$ is the reduced Planck constant and $v_{F} \approx 10^{6} \mathrm{~m} / \mathrm{s}$ is the Fermi velocity, $k^{\prime}$ is the momentum near the Dirac point, and $n$ is the charge density in graphene. The presence of 2D Dirac fermions in graphene and the square root dependence was observed in two experiments for the graphene cyclotron mass and the half-integer quantum Hall effect $[78,79]$. Charge carriers near 
Dirac points behave like relativistic particles ideally transporting with Fermi velocity, which is theoretically $\sim 300$ times slower than the speed of light [78].

Another extraordinary feature of graphene is that there is no energy gap between conduction and valence bands at Dirac points. As such, graphene can be considered as a semiconductor with no bandgap, or a semi-metal with no band overlap. If no external perturbation and no kinetic energy $(\mathrm{T}=0 \mathrm{~K})$ are present, the Fermi level, $E_{F}$ (or chemical potential, $\mu_{c}$ ) is exactly at the touching point between valence and conduction bands. At these points, the electron density is equal to the hole density, corresponding to the minimum value of the total carrier density and the so called charge neutrality point (CNP). The electric field can modulate the density of states and switch the device from low conductivity states near the Dirac point to high conductivity states elsewhere.

Due to the gapless nature of the dispersion, the number of charge carriers can be continuously changed by the electric field effect, adding either electrons or holes to the system, as sketched in Fig. 2.4(b). It can be observed that the electrons behave exactly the same as holes due to the symmetric conduction and valence band structures. Graphene shows perfect ambipolar electric field effect so that its charge carriers parity can be tuned to be either $\mathrm{n}$ - or $\mathrm{p}$ - type by applying electric field with different polarity. Interestingly, it is possible to achieve different carrier parity by varying the DC bias voltage, i.e. the perpendicular electric field, unlike traditional silicon technology wherein the materials need to be actually doped by other materials.

At the CNP, there is still a finite amount of current flowing through the low conduction state near the Dirac point, resulting in a non-negligible dark current for transistor applications. Furthermore, the charge density never vanishes completely due to the existence of electron-hole puddles on the $\mathrm{SiO}_{2}$ substrate, leading to minimum conductance on the order of $\sim e^{2} / h$ [80]. 
Scanning tunneling microscopy (STM) measurements show that local charge fluctuations of graphene on flat $\mathrm{hBN}$ are much smaller than on $\mathrm{SiO}_{2}$ substrate [81]. Electron transport in graphene has low scattering rate due to intrinsic phonons and thus the mobility at room temperature can reach to above $100000 \mathrm{~cm}^{2} \mathrm{~V}^{-1} \mathrm{~s}^{-1}$ on a device encapsulated between two layers of hBN [82]. This value is far beyond the mobility of graphene on $\mathrm{SiO}_{2}$ which is on the order of few thousands $\mathrm{cm}^{2} \mathrm{~V}^{-}$ ${ }^{1} \mathrm{~S}^{-1}[83]$.

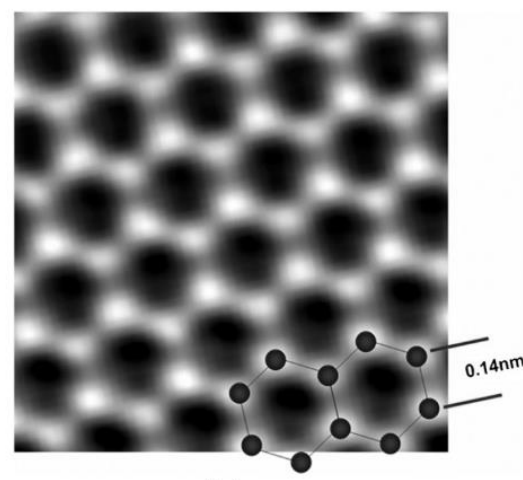

(a)

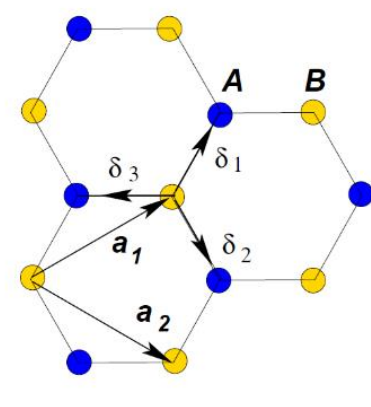

(b)

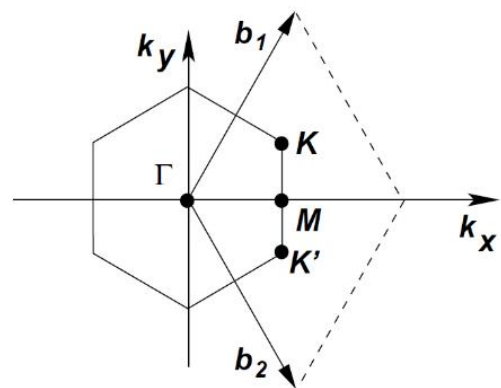

(c)

Figure 2.3: (a) Atomic-resolution image of graphene sheet showing individual carbon atoms in white. The figure is adopted by permission from [3]; (b) 2D hexagonal lattice of graphene in real space; (c) First Brillouin zone in momentum space. The figures are adopted by permission from [4]. The permission letter is attached in Appendix B.

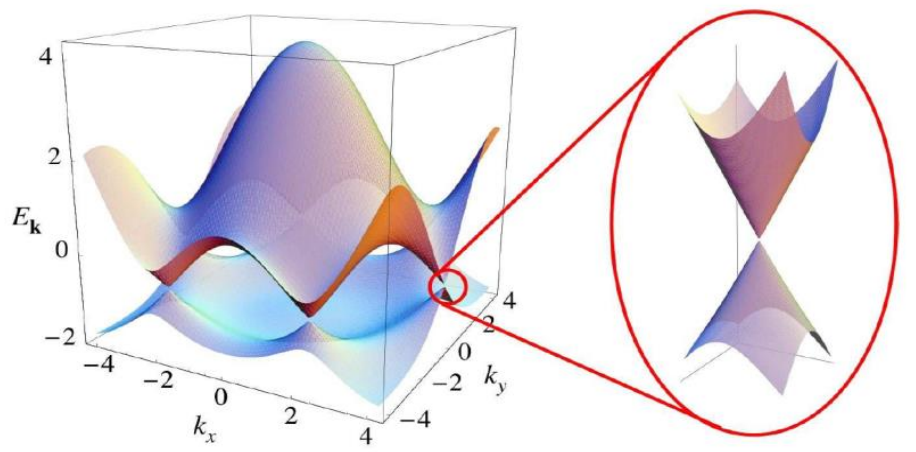

(a)

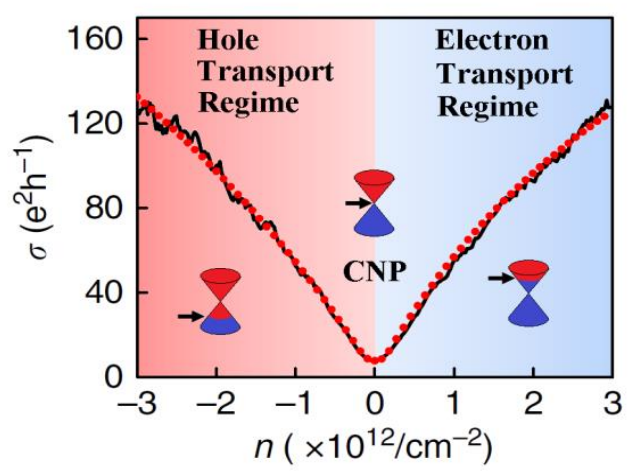

(b)

Figure 2.4: (a) Full dispersion from tight binding calculation and zoom on the low energy region at one of the K points, the so called Dirac points, adopted by permission from [4]; (b) Conductivity of graphene as a function of carrier density at room temperature. The black line of the experimental data is matched with red dots, obtained by a Boltzmann model of conductivity [5]. The cone-like dispersion as well as the positions of Fermi level (arrow) corresponding to different regions of operation are also shown. The permission letter is attached in Appendix B. 


\subsection{Photonics properties}

A 2D graphene sheet can physically be considered as one layer of 3Dbulk graphite, but it possesses distinct properties for photonic applications compared to traditional semiconductors. First of all, the zero bandgap and linear dispersion of graphene result in very broadband strong absorptance. The linear dispersion of Dirac fermions implies that there will always be an electronhole pair for broadband illumination, which is very different from semiconductors with bandgap and parabolic dispersion relations.

The optical absorption of suspended graphene has been measured to be $\sim 2.3 \%$ across visible and near-infrared (NIR) frequencies [6]. The value is equal to the universal constant $\pi \alpha$, where $\alpha=e^{2} /\left(4 \pi \varepsilon_{0} \hbar c\right) \approx 1 / 137$ is the fine structure constant [84]. The parameter describes coupling between light and relativistic electrons and is traditionally associated with quantum electrodynamics. The obtained value of optical absorption is remarkable for a one-atom thick material indicating that graphene can absorb a significant fraction of incident light. Figure 2.5(a) shows the photograph of a $50 \mathrm{~mm}$ aperture partially covered by suspended graphene and its bilayer [6]. The line scan across the image shows the changes in the intensity of transmitted white light. Graphene's absorption is measured close to $2.3 \%$ so that the opacity is almost independent of wavelength for visible illumination, as shown in Fig. 2.5(b). The inset shows that increasing the number of graphene layers by one enhances the absorption of the structure by $\sim 2.3 \%$. As such, the optical absorption can be increased by stacking few layers of graphene (FLG) with approximately the same ratio per layer of graphene. However, such structure needs to be isolated electrically by using dielectric insulators such as $\mathrm{hBN}$ to prevent the formation of graphite. 


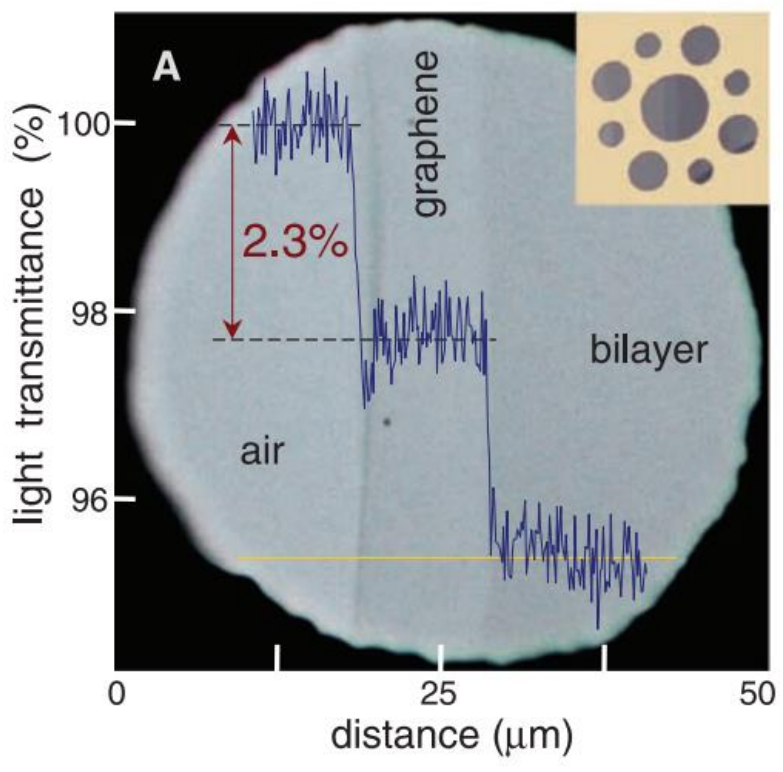

(a)

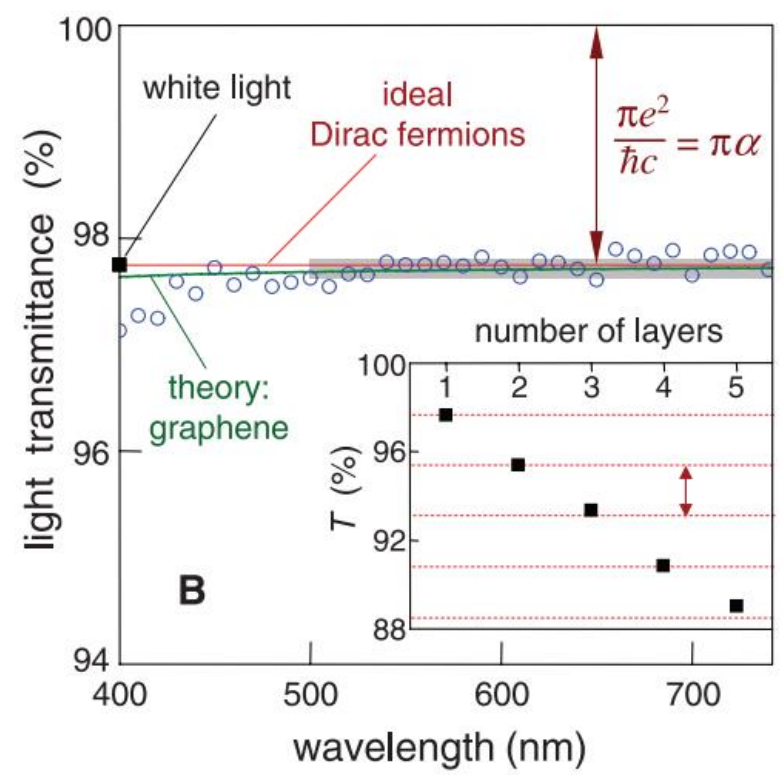

(b)

Figure 2.5: (a) Photograph of a 50-mm aperture partially covered by graphene and its bilayer. The line scan profile shows the intensity of transmitted white light along the yellow line. The inset is a 20- $\mu \mathrm{m}$-thick metal support structure with apertures of 20,30 , and $50 \mu \mathrm{m}$ in diameter with graphene placed over them. (b) Transmittance spectrum of single layer graphene (open circles). Slightly lower transmittance for $\lambda<500 \mathrm{~nm}$ is probably due to hydrocarbon contamination. The red line is the transmittance $T=(1+0.5 \pi \alpha)^{-2}$ expected for two-dimensional Dirac fermions, whereas the green curve takes into account the nonlinearity and triangular warping of graphene's electronic spectrum. The gray area indicates the standard error for measurements. The inset shows the transmittance of white light as a function of the number of graphene layers (squares). The dashed lines correspond to an intensity reduction by $\pi \alpha$ with each added layer. The figure is adopted by permission from [6]. Permission letter is attached in Appendix B.

\subsection{Graphene photonics applications}

As an atomic-thick material, graphene exhibits wavelength-independent absorption when the optical energy is below $3 \mathrm{eV}$, as well as complete transparency when the optical energy is smaller than double the Fermi level. These properties make graphene a promising candidate for many controllable photonic devices as shown in Fig. 2.6. In this section, an overview of potential graphene photonics applications is presented together with the expected time for their realization. 


\section{Transparent conductive films and passive photonic devices}

Graphene is expected to substitute indium tin oxide (ITO) [85] in transparent conductive films used in devices such as LEDs and OLEDs, solar cells, touchscreens, smart windows, and LCD films [86, 87]. For these applications, graphene has superior characteristics such as high conductivity, mechanical flexibility, and optical transparency. Graphene provides not only larger transmittance than ITO (about 97\% compared to 90\%), but is also less expensive and less brittle than ITO. Recent research is focused on decreasing the sheet resistance of graphene by external doping to make it a competitive alternative for these applications [88]. Another application of graphene is for shielding electromagnetic radiation in microwave and terahertz ranges. At these frequencies, five layers of doped graphene can provide $\sim 20 \mathrm{~dB}$ shielding efficiency [89], while millimeter thicknesses of traditional absorbers, such as ferrite or metal nanocomposites, are needed for such an effective shielding [90, 91]. As shown in Fig. 2.6, a graphene-based foldable touchscreen is expected to come into play as early as in 2020 [7].

\section{Photodetectors}

The spectral range and maximum bandwidth of traditional photodetectors, based on silicon, germanium, or compound semiconductors, are limited by the bandgap energy and carrier transit times of materials [92]. On the other hand, due to the absence of a bandgap and the high carrier mobility of graphene, graphene-based photodetectors can, in principle, be used in a wide spectral range from ultraviolet to terahertz frequencies. Ultrafast extraction of photo-generated carriers is enabled by the high carrier mobility in graphene, resulting in extremely high bandwidth operation. As such, the transit-time-limited bandwidth of graphene photodetectors can possibly reach $1.5 \mathrm{THz}$ [93], while that of InGaAs photodetectors is limited to $150 \mathrm{GHz}$ [92]. It has been already demonstrated $[93,94]$ that infrared light pulses irradiated at the rate of $10 \mathrm{GHz}$ and $40 \mathrm{GHz}$ can 
be reliably detected by graphene-metal junctions, demonstrating the suitability of graphene for high-speed optical communications in the near future. However, the atomic thinness of graphene limits its absorption, so that the maximum responsivity of a graphene photodetector is low, much smaller than the required value of $1 \mathrm{AW}^{-1}$ for effective detection [94].

There are several possible ways to increase the sensitivity of graphene photodetectors such as using plasmonic nanostructures for the enhancement of the local optical electric field [95], integrating it with a waveguide to increase the light-graphene interaction length [96], or simply increasing the number of graphene layers in an aperiodic multilayer structure, as proposed in the current thesis. For instance, at the resonant wavelength, a 20 -fold enhancement of the photocurrent was achieved by incorporating a graphene layer inside Fabry-Perot microcavities [97] or a 60\% absorption by utilizing Bragg reflectors [98]. It is expected that a graphene photodetector with a bandwidth of over $100 \mathrm{GHz}$ will be competitive after 2020 [7], as shown in Fig. 2.6.

\section{Optical modulator}

Optical modulators with high speed, small footprint, and high bandwidth are highly desirable for optical interconnects to encode transmission data by altering the light properties [99]. However, conventional electro-absorption modulators have limited operation bandwidth due to the slow switching time of silicon, which decreases their bandwidth to usually less than $\sim 50 \mathrm{GHz}$ [100]. Graphene absorption modulators have not only ultrafast response in absorbing a small amount of incident light over ultrawide range of wavelengths, but also the interband transitions of photo-generated electrons can be modulated over broad spectral ranges by tuning the Fermi level of graphene layers [101]. Despite the strong light-graphene interaction, the absorption of a light beam by a single graphene layer is insufficient so that it is usually coupled with a silicon waveguide to increase the absorption in the near infrared range [102]. The optical resonance of a photonic 
crystal nanocavity can be modulated by tuning the Fermi level in graphene layers, by reducing the resistance so as to reduce RC delay times, and by modifying the structures [103, 104], so that operation bandwidths in the terahertz range are expected. However, such developments are not expected before 2020, as shown in Fig. 2.6 [7].

\section{Mode-locked laser}

Graphene is also promising to find an application as a saturable absorber in fiber and laser technologies, wherein the absorption of light by a material decreases with increasing light intensity [105]. Saturated absorption can be observed in widely used semiconductors at very high optical intensities [106]; however, graphene reaches saturation at a lower intensity, by absorbing a significant amount of light energy per unit thickness [107, 108]. As such, graphene-saturable absorbers demonstrate ultrafast carrier relaxation time, controllable modulation depth, high damage threshold, high thermal conductivity, high stability, and wide tunability of the spectral absorption range $[6,109,110]$. Graphene-based tunable mode-locked lasers are expected to be commercialized by 2020 [7]. A graphene-saturable absorber could also enable an ultrafast passively mode-locked laser to operate at many different wavelengths for various applications such as spectroscopy, optical interconnects [111], bio-medicine [112], and material micromachining [113]. However, this application is expected to be demonstrated only after developing highly integrated optical interconnects around the late 2020s, as shown in Fig. 2.6 [7].

\section{THz wave generator}

$\mathrm{THz}$ wave generators are needed for various applications such as medical imaging, chemical sensors, and security devices. Graphene can be used as a gain medium to generate stimulated emission by optical pumping. However, as graphene has the same diffusion times for electrons and holes, the formation of a dipole may not be sufficient to overcome the stimulated 
emission threshold for continuous THz wave generation [114]. To generate waves in this range, it has been suggested to accelerate the carriers in a single layer or multiple layers of graphene using a femtosecond laser pulse field. However, due to the weak intensity of the generated waves in comparison with III-V semiconductors, it is expected that graphene-based THz-wave generators are unlikely to emerge before 2030, as shown in Fig. 2.6 [7].

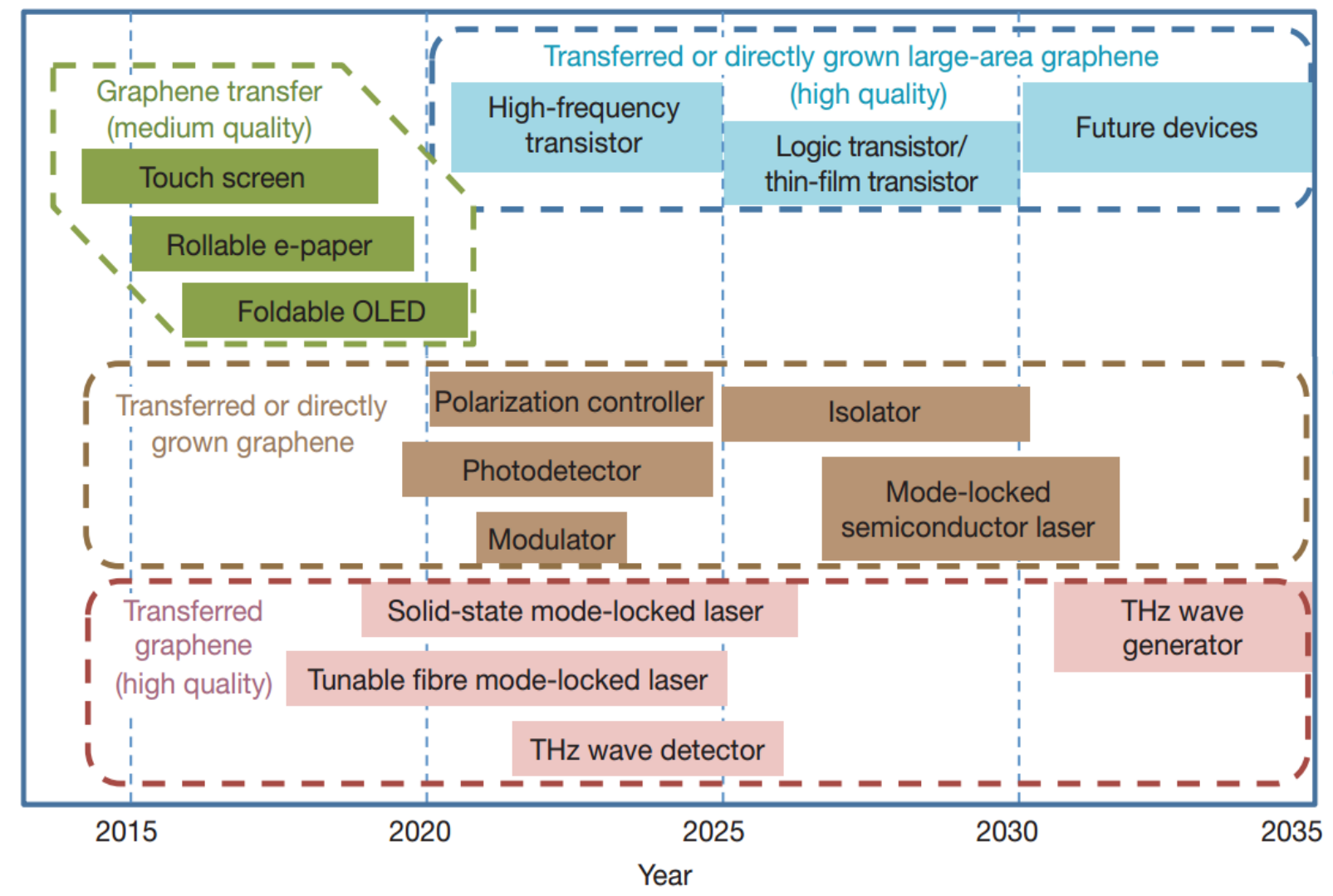

Figure 2.6: Graphene photonic applications in display technology (green), electronic devices (blue), as well as optical devices (pink), and interconnects (brown). Application timelines are based on projections of products requiring advanced materials such as graphene, which can be enabled by continuous advances in graphene technology. The figure gives an indication of when a functional device prototype could be expected based on device roadmaps and the development schedules of industry leaders [7]. The quality of graphene and the required synthesis methods are shown for each category of application. The details are beyond the scope of this thesis. The figure is adopted by permission from [7]. The permission letter is attached in Appendix B. 


\section{Optical polarization controller}

Optical polarization controllers are crucial passive components to manipulate the polarization properties of photons. The excitation of Dirac fermions in graphene enables the differential attenuation of the transverse magnetic mode, leading to an excellent extinction ratio of $\sim 27 \mathrm{~dB}$ for broadband communications [115]. The hybrid graphene-based optical polarizer, in which a high-quality large-size graphene layer is integrated with an optical fiber, is expected to be fabricated by 2020 [7]. 


\section{CHAPTER 3 \\ PHYSICAL MODELS AND SIMULATION METHOD}

\subsection{Introduction}

In this chapter, infrared thermal radiation is first discussed using Planck's blackbody law, followed by Kirchoff's law of thermal radiation. Then, transfer matrix formalism is described that relates the electric field at air to the electric field at substrate in an aperiodic multilayer structure. Next section is dedicated to the brief description of a hybrid optimization method that is used to find the thicknesses of layers in the aperiodic multilayer structure. Finally, the Kubo formalism is presented that relates the optical conductivity, and therefore the refractive index, to the chemical potential in graphene.

\subsection{Thermal emitter / Blackbody radiation}

The electromagnetic spectrum is the range of all possible wavelengths, ranging from gamma ray radiation down to radio wave radiation, as shown in Fig. 3.1. All objects that are warmer than their surroundings emit photons within a certain range of wavelengths. This range is not a characteristic of the materials in the object and exclusively depends on the surface temperature of the object that is emitting the electromagnetic radiation. As such, gamma and Xrays radiation are emitted by very hot objects of temperature of a million Kelvin, while photons with longer wavelengths in the infrared and radio wavelength range are emitted by cooler objects. The higher the temperature, the more intense is the emitted radiation. Also, the wavelength of maximum emission shifts to shorter wavelengths by increasing the temperature of the object.

To find the power radiated per unit area and wavelength, one can calculate the Planck blackbody spectrum (in units of Watts per square meter per nanometer) as follows: 


$$
B(\lambda, T)=\frac{2 h c^{2}}{\lambda^{5}} \frac{1}{e^{\frac{h c}{\lambda K_{B} T}}-1},
$$

where $K_{B}$ is the Boltzmann constant, $h$ is Plank's constant, $c$ is the speed of light, $T$ is the ambient temperature, and $\lambda$ is the wavelength. An idealized blackbody absorbs all radiation that falls into the range enforced by the temperature of the object. Kirchoff's law of thermal radiation states that the emittance and absorptance of an object must be equal for a given frequency, direction, and polarization under the condition of thermal equilibrium.

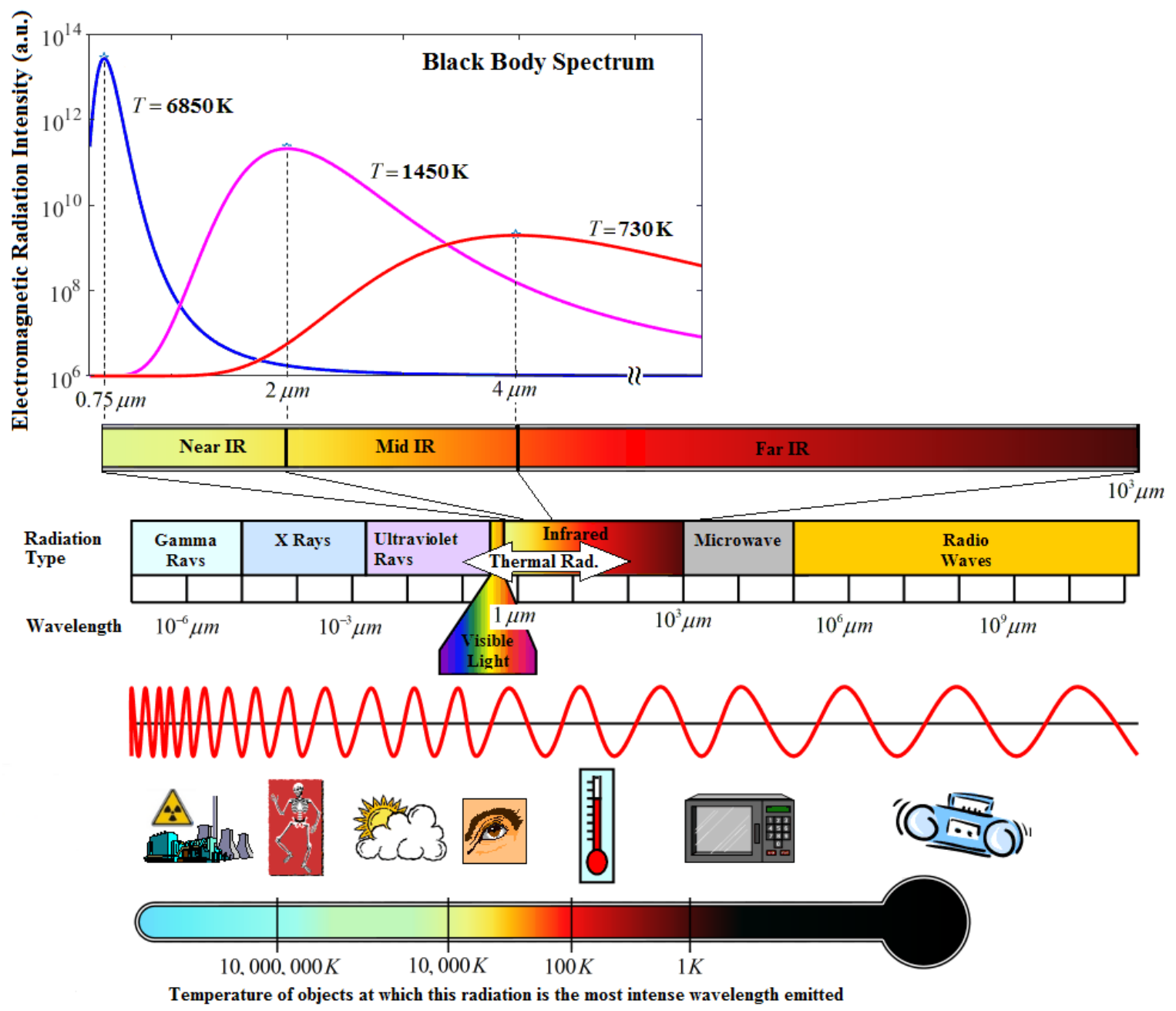

Figure 3.1: Electromagnetic spectrum as well as the thermal radiation spectrum including the blackbody radiation at near infrared and mid-infrared wavelengths. The figure is reproduced from another figure in the public domain. 
As such, the normalized power radiated per unit area and unit wavelength by a nonblackbody in the normal direction as a function of wavelength and temperature can be calculated as follows:

$$
\bar{\mu}(\lambda)=\frac{\in_{\text {Total }}(\lambda) B(\lambda, T)}{\max _{\lambda}[B(\lambda, T)]},
$$

where $\epsilon_{\text {Total }}(\lambda)=\left[\epsilon_{T E}(\lambda)+\epsilon_{T M}(\lambda)\right] / 2$ is the averaged emittance from both TE and TM polarizations in the normal direction. The value of $\bar{\mu}(\lambda)$ indicates how well the multilayer structure emits photons at a given wavelength in the normal direction, as explained in next chapters.

\subsection{Transfer matrix formalism}

Photonic nanostructures may be computationally designed to affect the motion of photons in a desired way so that a specific application is achieved. Such simulations involve solving the electromagnetic equations (Maxwell's equations) in one, two, or three dimensions by using computational methods such as the transfer matrix, the finite-difference frequency-domain (FDFD) method, and the finite-difference time-domain (FDTD) method [116]. The simulation of 2D and 3D structures is computationally intensive, but simulating $1 \mathrm{D}$ structures can provide a proof of concept to design and implement more complex structures.

Different approaches are possible to obtain the absorption, transmission, and reflection coefficients in a nanophotonic structure. One of the more elegant approaches to calculate the field distribution in aperiodic multilayer structures is by employing the transfer matrix method, leading to information about the absorption, transmission, and reflection properties of the structure. Figure 3.2 is a schematic showing a general multilayer structure having $m$ layers between a semi-infinite transparent ambient and a semi-infinite substrate. Consider a plane wave incident from air on the 
left side of the structure so that the optical waves propagate along the x-axis. Each layer $j$ ( $j=1,2, \ldots, m)$ has a thickness $a_{j}$ and its optical properties are described by its complex index of refraction $\tilde{n}_{j}(\lambda)=\eta_{j}(\lambda)+i \kappa_{j}(\lambda)$ which is a function of wavelength of the incident light $\lambda$. This stratified structure with isotropic and homogeneous media and parallel-plane interfaces can be described by $2 \times 2$ matrices, since the equations governing the electric field propagation are linear and the tangential components of the electric and magnetic fields are continuous [117, 118]. For normally incident plane waves, the TM and TE modes have the same behavior.

For TM polarization, the transverse components of the electric filed, $E_{z}$, and magnetic field, $H_{y}$, in each layer can be described by:

$$
\begin{gathered}
E_{z}(x, y)=\left(A_{j} e^{i k_{j} x}+B_{j} e^{-i k_{j} x}\right) e^{i k_{y} y} \equiv E_{z}(x) e^{i k_{y} y}, \\
H_{y}(x, y)=\frac{-c k_{j}}{Z_{j} \omega}\left(A_{j} e^{i k_{j} x}-B_{j} e^{-i k_{j} x}\right) e^{i k_{y} y} \equiv H_{y}(x) e^{i k_{y} y},
\end{gathered}
$$

where $k_{j}$ is the propagation constant of the waves in the x-direction in the $j^{\text {th }}$ layer and $k_{y}$ is the propagation constant in the y-direction for all layers. $A_{j}$ and $B_{j}$ are the amplitudes of the forward and backward propagating waves in the $j^{\text {th }}$ layer, respectively. $Z_{j}=\sqrt{\mu_{0} / \varepsilon_{j}}$ is the free-space impedance, $c$ is speed of light in free space, $\mu_{0}$ is the free-space magnetic permeability, and $\varepsilon_{j}$ is the dielectric permittivity of the material in the $j^{\text {th }}$ layer.

By applying the boundary conditions at the interface between air and the first layer of the structure, one obtains

$$
\left[\begin{array}{c}
E_{z} \\
Z_{0} H_{y}
\end{array}\right]=\left[\begin{array}{cc}
1 & 1 \\
\frac{-c k_{1}}{\omega} & \frac{c k_{1}}{\omega}
\end{array}\right]\left[\begin{array}{l}
A_{1} \\
B_{1}
\end{array}\right] .
$$


Then, by applying the boundary conditions at the second interface, the matrix relating the transverse fields at the two interfaces can be obtained as follows:

$$
\begin{aligned}
{\left[\begin{array}{c}
E_{z} \\
Z_{0} H_{y}
\end{array}\right] } & =\left[\begin{array}{cc}
1 & 1 \\
\frac{-c k_{1}}{\omega} & \frac{c k_{1}}{\omega}
\end{array}\right]\left[\begin{array}{cc}
e^{i k_{1} X_{1}} & 0 \\
0 & e^{-i k_{1} X_{1}}
\end{array}\right]\left[\begin{array}{c}
A_{1} \\
B_{1}
\end{array}\right] \\
& =\left[\begin{array}{cc}
1 & 1 \\
\frac{-c k_{1}}{\omega} & \frac{c k_{1}}{\omega}
\end{array}\right]\left[\begin{array}{cc}
e^{i k_{1} X_{1}} & 0 \\
0 & e^{-i k_{1} X_{1}}
\end{array}\right]\left[\begin{array}{cc}
1 & 1 \\
\frac{-c k_{1}}{\omega} & \frac{c k_{1}}{\omega}
\end{array}\right]\left[\begin{array}{c}
E_{z} \\
Z_{0} H_{y}
\end{array}\right]_{X_{0}} \\
& =\left[\begin{array}{cc}
\cos \left(k_{1} a_{1}\right) & -i \sin \left(k_{1} a_{1}\right) \\
-i \sin \left(k_{1} a_{1}\right) \frac{c k_{1}}{\omega} & \cos \left(k_{1} a_{1}\right)
\end{array}\right]\left[\begin{array}{c}
E_{z} \\
Z_{0} H_{y}
\end{array}\right]_{X_{0}} .
\end{aligned}
$$

The fields in the first layer can be related to those in the last one as follows:

$$
\left[\begin{array}{c}
E_{z} \\
Z_{0} H_{y}
\end{array}\right]_{X_{m+1}}=T_{r}\left[\begin{array}{c}
E_{z} \\
Z_{0} H_{y}
\end{array}\right]_{X_{0}},
$$

where $T_{r}$ is the transfer matrix obtained by multiplying the matrices of all layers in the structure as follows:

$$
\begin{aligned}
& T_{r}=\left[\begin{array}{cc}
\cos \left(k_{m+1} a_{m+1}\right) & -i \sin \left(k_{m+1} a_{m+1}\right) \\
-i \sin \left(k_{m+1} a_{m+1}\right) \frac{c k_{m+1}}{\omega} & \cos \left(k_{m+1} a_{m+1}\right)
\end{array}\right] \times \ldots \\
& {\left[\begin{array}{cc}
\cos \left(k_{2} a_{2}\right) & -i \sin \left(k_{2} a_{2}\right) \\
-i \sin \left(k_{2} a_{2}\right) \frac{c k_{2}}{\omega} & \cos \left(k_{2} a_{2}\right)
\end{array}\right]\left[\begin{array}{cc}
\cos \left(k_{1} a_{1}\right) & -i \sin \left(k_{1} a_{1}\right) \\
-i \sin \left(k_{1} a_{1}\right) \frac{c k_{1}}{\omega} & \cos \left(k_{1} a_{1}\right)
\end{array}\right] .}
\end{aligned}
$$

Thus, the field at each position $\mathrm{x}$ can be calculated. Considering the boundary condition for the last layer (substrate), one can derive the relation between the reflection and transmission coefficients as follows:

$$
\left[\begin{array}{l}
1 \\
r
\end{array}\right]=\left[\begin{array}{cc}
1 & 1 \\
\frac{-c k_{1}}{\omega} & \frac{c k_{1}}{\omega}
\end{array}\right]^{-1} T_{r}^{-1}\left[\begin{array}{cc}
1 & 1 \\
\frac{-c k_{1}}{\omega} & \frac{c k_{1}}{\omega}
\end{array}\right]\left[\begin{array}{l}
t \\
0
\end{array}\right],
$$


where $r$ and $t$ are the reflection and transmission coefficients of the multilayer structure. By calculating the reflection and transmission, the absorptance, $A$, of the structure can be obtained as $A=1-|t|^{2}-|r|^{2}$

One can find a relation between the electric field at any point in the system and the corresponding absorption in the layers of the multilayer structure [119]. As shown in Fig. 3.2, the total electric field at any point in the system can be decomposed into two components. One component propagating in the positive $x$ direction and another in the negative $x$ direction, which at a position $x$ in layer $j$ are denoted as $E_{j}^{+}(x)$ and $E_{j}^{-}(x)$, respectively. Then, each interface in the structure can be described by the interface matrix between layers $j$ and $j-1$ as follows:

$$
I_{j, j-1}=\frac{1}{t_{j, j-1}}\left[\begin{array}{cc}
1 & r_{j, j-1} \\
r_{j, j-1} & 1
\end{array}\right],
$$

where $r_{j, j-1}$ and $t_{j, j-1}$ are the Fresnel complex reflection and transmission coefficients at the interface. For light with the electric field perpendicular to the plane of incidence (corresponding to s-polarized or TE waves) and parallel to the plane of incidence (corresponding to p-polarized or TM waves), the Fresnel complex reflection and transmission coefficients can be obtained as follows:

$$
\begin{gathered}
r_{j, j-1}=\frac{q_{j}-q_{j-1}}{q_{j}+q_{j-1}}, \quad t_{j, j-1}=\frac{2 q_{j}}{q_{j}+q_{j-1}}, \\
r_{j, j-1}=\frac{\tilde{n}_{j-1}^{2} q_{j}-\tilde{n}_{j}^{2} q_{j-1}}{\tilde{n}_{j-1}^{2} q_{j}+\tilde{n}_{j}^{2} q_{j-1}}, \quad t_{j, j-1}=\frac{2 \tilde{n}_{j} \tilde{n}_{j-1} q_{j}}{\tilde{n}_{j-1}^{2} q_{j}+\tilde{n}_{j}^{2} q_{j-1}},
\end{gathered}
$$

where $q_{j}=\tilde{n}_{j} \cos \phi_{j}=\left[\tilde{n}_{j}^{2}-\eta_{0}^{2} \sin \phi_{0}\right]^{1 / 2}, \eta_{0}=1$ is the refractive index of air, $\phi_{0}$ is the angle of incidence in air, and $\phi_{j}$ is the angle of refraction in layer $j$. The phase matrix describing the propagation through layer $j$ is described by, 


$$
L_{j}=\left[\begin{array}{cc}
e^{-i \xi_{j} a_{j}} & 0 \\
0 & e^{i \xi_{j} a_{j}}
\end{array}\right],
$$

where $\xi_{j}=2 \pi q_{j} / \lambda$, and $\xi_{j} a_{j}$ is the layer phase thickness corresponding to the phase change that the wave experiences when it traverses layer $j$. Similar to Eq. (3.6), the transfer matrix can be expressed for the two components of the electric field as follows:

$$
\left[\begin{array}{c}
E_{0}^{+} \\
E_{0}^{-}
\end{array}\right]=S\left[\begin{array}{c}
E_{m+1}^{+} \\
E_{m+1}^{-}
\end{array}\right],
$$

where $S$ is the total system transfer matrix that relates the electric field at air to the electric field at the substrate as follows:

$$
S=\left[\begin{array}{ll}
S_{11} & S_{12} \\
S_{21} & S_{22}
\end{array}\right]=\left(\prod_{v=1}^{m} I_{(v-1) v} L_{v}\right) I_{m(m+1)} .
$$

As the light is incident from the air side in the positive $x$ direction, there is no wave propagating in the negative $x$ direction inside the substrate and thereby $E_{m+1}^{-}=0$. Thus, the complex reflection and transmission coefficients in Eqs. (3.10) and (3.11) can be expressed in term of the matrix elements of the total system transfer matrix in Eq. (3.14) as follows:

$$
r=\frac{E_{0}^{-}}{E_{0}^{+}}=\frac{S_{21}}{S_{11}}, \quad t=\frac{E_{m+1}^{+}}{E_{0}^{+}}=\frac{1}{S_{11}} .
$$

The internal electric field in layer $j$ can be calculated by dividing the layered structure into two subsets, separated by layer $j$ in Fig. 3.2, which means that the total system transfer matrix can be written as follows:

$$
S=S_{j}^{\prime} L_{j} S_{j}^{\prime \prime}
$$

The partial system transfer matrices for layer $j, S_{j}^{\prime}$ and $S_{j}^{\prime \prime}$, can be defined as follows: 


$$
\begin{aligned}
& {\left[\begin{array}{l}
E_{0}^{+} \\
E_{0}^{-}
\end{array}\right]=S_{j}^{\prime}\left[\begin{array}{c}
E_{j}^{+} \\
E_{j}^{\prime-}
\end{array}\right], \quad S_{j}^{\prime}=\left[\begin{array}{ll}
S_{j 11}^{\prime} & S_{j 12}^{\prime} \\
S_{j 21}^{\prime} & S_{j 22}^{\prime}
\end{array}\right]=\left(\prod_{v=1}^{j-1} I_{(v-1) v} L_{v}\right) \cdot I_{(j-1) j},} \\
& {\left[\begin{array}{l}
E_{j}^{\prime \prime+} \\
E_{j}^{\prime \prime}
\end{array}\right]=S_{j}^{\prime \prime}\left[\begin{array}{l}
E_{m+1}^{+} \\
E_{m+1}^{-}
\end{array}\right], \quad S_{j}^{\prime \prime}=\left[\begin{array}{ll}
S_{j 11}^{\prime \prime} & S_{j 12}^{\prime \prime} \\
S_{j 21}^{\prime \prime} & S_{j 22}^{\prime \prime}
\end{array}\right]=\left(\prod_{v=j+1}^{m} I_{(v-1) v} L_{v}\right) \cdot I_{m(m+1)},}
\end{aligned}
$$

where $E_{j}^{\prime+}$ and $E_{j}^{\prime-}$ refer to the left boundary of layer $j$ and $E_{j}^{\prime \prime+}$ and $E_{j}^{\prime \prime-}$ refer to the right boundary of layer $j$. Similarly, the complex reflection and transmission coefficients for layer $j$ are given by:

$$
\begin{aligned}
& r_{j}^{\prime}=\frac{S_{j 21}^{\prime}}{S_{j 11}^{\prime}}, \quad t_{j}^{\prime}=\frac{1}{S_{j 11}^{\prime}}, \\
& r_{j}^{\prime \prime}=\frac{S_{j 21}^{\prime \prime}}{S_{j 11}^{\prime \prime}}, \quad t_{j}^{\prime \prime}=\frac{1}{S_{j 11}^{\prime \prime}} .
\end{aligned}
$$

Combining Eqs. (3.15)-(3.20), the internal transfer coefficients which relate the incident plane wave to the internal electric field propagating in the negative $x$ and positive $x$ directions in layer $j$ at its left boundary can be derived as follows:

$$
\begin{gathered}
t_{j}^{+}=\frac{E_{j}^{+}}{E_{0}^{+}}=\frac{t_{j}^{\prime}}{1-r_{j-}^{\prime} r_{j}^{\prime \prime} \cdot e^{i 2 \xi_{j} a_{j}}}, \\
t_{j}^{-}=\frac{E_{j}^{-}}{E_{0}^{+}}=\frac{t_{j}^{\prime} r_{j}^{\prime \prime} \cdot e^{i 2 \xi_{j} a_{j}}}{1-r_{j-}^{\prime} r_{j}^{\prime \prime} \cdot e^{i 2 \xi_{j} a_{j}}}=t_{j}^{+} r_{j}^{\prime \prime} \cdot e^{i 2 \xi_{j} a_{j}},
\end{gathered}
$$

where $r_{j-}^{\prime}=-S_{j 12}^{\prime} / S_{j 11}^{\prime}$. Using Eqs. (3.21) and (3.22), one can obtain the total electric field in an arbitrary plane in layer $j$ at a distance $x\left(0 \leq x \leq a_{j}\right)$ to the right of its left boundary in terms of the incident plane wave $E_{0}^{+}$as follows: 


$$
\begin{aligned}
& E_{j}(x)=E_{j}^{+}(x)+E_{j}^{-}(x) \\
& =\left[t_{j}^{+} e^{i \xi_{j} x}+t_{j}^{-} e^{-i \xi_{j} x}\right] E_{0}^{+} \\
& =t_{j}^{+}\left[e^{i \xi_{j} x}+r_{j}^{\prime \prime} e^{i \xi_{j}\left(2 a_{j}-x\right)}\right] E_{0}^{+} .
\end{aligned}
$$

The above expression can also be expressed in terms of the matrix elements of the partial system transfer matrices as follows:

$$
E_{j}(x)=\frac{S_{j 11}^{\prime \prime} \cdot e^{-i \xi_{j}\left(a_{j}-x\right)}+S_{j 21}^{\prime \prime} \cdot e^{-i \xi_{j}\left(a_{j}-x\right)}}{S_{j 11}^{\prime} S_{j 11}^{\prime \prime} \cdot e^{-i \xi_{j} a_{j}}+S_{j 12}^{\prime} S_{j 21}^{\prime \prime} \cdot e^{i \xi_{j} a_{j}}} E_{0}^{+} .
$$

Thus, the power as a function of $x$ within the structure can be calculated to locate the areas of high and low loss within the structure.

The number of excited states at a given position in a structure is directly dependent on the electromagnetic power absorbed by the material. Thus, the time average of the energy dissipated per second in layer $j$ at position $x$ at normal incidence is given by [119],

$$
Q_{j}(x)=\frac{1}{2} c \varepsilon_{0} \alpha_{j} \eta_{j}\left|E_{j}(x)\right|^{2}
$$

This means that the energy absorbed at position $x$ in the layered structure is proportional to the product of the amplitude of the electric field squared $\left|E_{j}(x)\right|^{2}$, the real part of refractive index $\eta_{j}$, and the absorption coefficient $\alpha_{j}=4 \pi k_{j} / \lambda$ at the actual position $x$. As such, the number of excited states in a layer is proportional to the number of absorbed photons and, hence, $|E|^{2}$ versus position $x$ in the film directly represents the production of excited states at each point. 


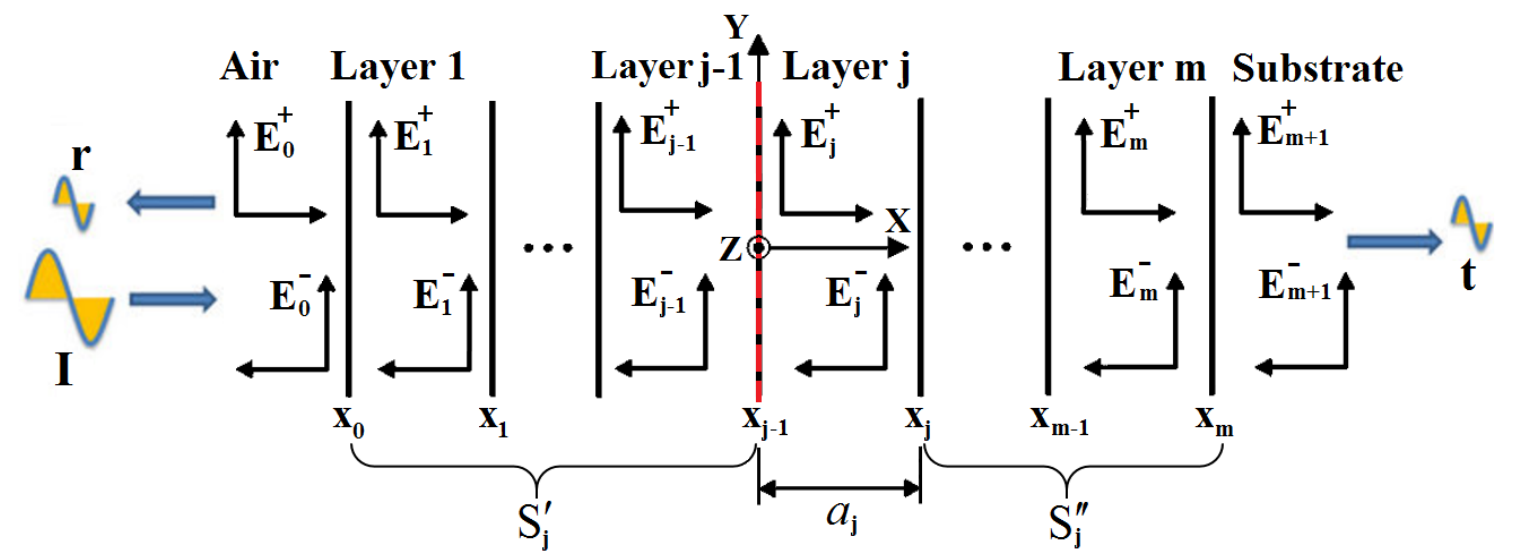

Figure 3.2: A multilayer structure having $m$ layers between a semi-infinite air region and a semiinfinite substrate. Each layer has a thickness of $a_{j}$ and its optical properties are described by its complex index of refraction. The optical electric field at any point in layer $j$ is represented by two components: one propagating in the positive and one in the negative $x$ direction, $E_{j}^{+}$and $E_{j}^{-}$, respectively.

\subsection{Genetic algorithm}

In order to find the optimum thicknesses of the layers in the aperiodic multilayer structure, a hybrid optimization method [120] consisting of a microgenetic global optimization algorithm coupled to a local optimization algorithm is employed. Using this algorithm, the optimized thicknesses for maximizing the absorption to the perfect value of unity can be found at a prespecified wavelength and zero bias condition $(\mu=0 \mathrm{eV})[121,122]$.

The genetic algorithm is an iterative optimization procedure which starts with a randomly selected population of potential solutions and gradually evolves toward improved solutions by applying the genetic operators which are patterned after the natural selection process. To begin the algorithm, a population of chromosomes is created by a random selection of values for the genes. Then the genetic algorithm proceeds to iteratively generate a new population by the crossover, mutation, and selection operators [34].

More specifically, this thesis made use of a microgenetic algorithm to avoid the problem of premature convergence, resulting in faster convergence to the near-optimal region [36, 123 , 
124]. Starting with a small population of random members, the microgenetic algorithm evolves and converges after a few generations. At this point, the genetic algorithm selects a new random population, and the evolution process restarts while keeping the best individual from the previously converged generation. In this genetic algorithm, a tournament selection is employed as the selection scheme so that a subpopulation of individuals is randomly chosen from the general population and then compete based on their fitness values. As such, the individual with the highest fitness value is selected as the winner of the tournament in a repeating process. Then, a pair of individuals is selected as the parents, and two offspring are created by the crossover operator so that they possess a combination of the chromosomes of their parents. As the microgenetic algorithm convergence is faster with uniform crossover [125], the uniform crossover is used rather than single-point crossover. The best individual from the previous population is chosen to pass on to the next corresponding to an elitist strategy [126]. It has been shown that this selection scheme converges more rapidly and operates more quickly with faster execution time when compared to other competing schemes [125].

\subsection{Optical conductivity model of graphene}

The refractive index is not well defined for $2 \mathrm{D}$ graphene because there is no rigorous definition for the induced polarization per unit volume. A more suitable physical quantity to describe the optical properties of graphene is the optical conductivity, also known as the carrier dynamic or AC conductivity of graphene. The optical conductivity is a complex number associated with the surface current induced in the $2 \mathrm{D}$ crystal by light [127]. The absorption is proportional to the real part of the optical conductivity [128], and the imaginary part is closely related to its behavior for application as a hyperbolic metamaterial. For instance, the sign of the imaginary part 
of the optical conductivity determines whether graphene supports transverse-electric (TE) or transverse-magnetic (TM) plasmons [129].

A large body of literature focuses on different techniques to model the complex conductivity of graphene [59, 130-133]. This section introduces and reviews the analytical expression for the complex conductivity of graphene. The response of graphene's optical conductivity to an external electric field was derived theoretically by several researchers $[58,130$, $134,135]$. Using the non-interacting linear response theory $[58,127]$, electrons are considered to move due to the applied electric field that is the sum of the external field and the self-consistent field induced by all the electrons. The cone-like dispersion near Dirac points is assumed, since the exact band structure results in only few percent difference in the conductivity at optical frequencies [127]. As a graphene sheet is an infinitesimally-thin, non-local two-sided surface in free space in the $x-y$ plane, one can obtain an extended non-local anisotropic model of conductivity using a magneto-optical surface complex conductivity tensor as follows $[132,136]$ :

$$
\stackrel{\leftrightarrow}{\sigma}\left(\omega, \mu_{c}\left(\mathbf{E}_{\mathbf{0}}\right), \Gamma, T, \mathbf{B}_{\mathbf{0}}\right)=x x \sigma_{x x}+x y \sigma_{x y}+y x \sigma_{y x}+y y \sigma_{y y},
$$

where $\omega$ is the radian frequency, $\mu_{c}$ is the chemical potential, $\Gamma$ is the charge particle scattering rate, $T$ is the temperature, and $\mathbf{E}_{\mathbf{0}}$ and $\mathbf{B}_{\mathbf{0}}$ are the DC electric and magnetic fields, respectively. The chemical potential can be controlled by chemical doping [137] or by applying DC bias field perpendicular to the graphene surface $\left(\mathbf{E}_{\mathbf{0}}=\hat{z} E_{0}\right)$ [63]. The geometry of graphene sheet implies that $\sigma_{x x}=\sigma_{y y}$ and $\sigma_{x y}=-\sigma_{y x}$, and thus Eq. (3.26) can be rewritten as follows:

$$
\stackrel{\leftrightarrow}{\sigma}\left(\omega, \mu_{c}\left(\mathbf{E}_{\mathbf{0}}\right), \Gamma, T, \mathbf{B}_{\mathbf{0}}\right)=\sigma_{d} \overleftrightarrow{\mathbf{I}}_{\mathrm{t}}+\sigma_{0} \overleftrightarrow{\mathbf{J}}_{\mathrm{t}}
$$


where $\overrightarrow{\mathbf{I}_{\mathrm{t}}}=x x+y y$ and $\overrightarrow{\mathbf{J}_{\mathrm{t}}}=x y-y x$ are the symmetric and antisymmetric dyads, respectively. $\sigma_{d}$ and $\sigma_{0}$ are the diagonal and off-diagonal (Hall) conductivities, respectively, in the presence of electric potential and magnetic bias fields, which can be obtained using the Kubo formula [138, 139] as follows:

$$
\begin{aligned}
\sigma_{d}=\frac{e^{2} v_{F}^{2}\left|e B_{0}\right| \hbar(\omega+i 2 \Gamma)}{i \pi} \\
\quad \times \sum_{n=0}^{\infty}\left\{\left(1-\frac{\Delta^{2}}{M_{n} M_{n+1}}\right) \frac{\left[n_{F}\left(M_{n}\right)-n_{F}\left(M_{n+1}\right)\right]+\left[n_{F}\left(-M_{n+1}\right)-n_{F}\left(-M_{n}\right)\right]}{\left(M_{n+1}-M_{n}\right)^{2}-(\omega+2 i \Gamma)^{2}}\right. \\
\quad \times \frac{1}{M_{n+1}-M_{n}}+\left(1+\frac{\Delta^{2}}{M_{n} M_{n+1}}\right) \\
\left.\quad \times \frac{\left[n_{F}\left(-M_{n}\right)-n_{F}\left(M_{n+1}\right)\right]+\left[n_{F}\left(-M_{n+1}\right)-n_{F}\left(M_{n}\right)\right]}{\left(M_{n+1}+M_{n}\right)^{2}-(\omega+2 i \Gamma)^{2}} \frac{1}{M_{n+1}+M_{n}}\right\},
\end{aligned}
$$

and

$$
\begin{aligned}
\sigma_{0}=- & \frac{e^{2} v_{F}^{2} e B_{0}}{\pi} \sum_{n=0}^{\infty}\left(\left[n_{F}\left(M_{n}\right)-n_{F}\left(M_{n+1}\right)\right]+\left[n_{F}\left(-M_{n+1}\right)-n_{F}\left(-M_{n}\right)\right]\right) \\
& \times\left\{\left(1-\frac{\Delta^{2}}{M_{n} M_{n+1}}\right) \frac{1}{\left(M_{n+1}-M_{n}\right)^{2}-(\omega+2 i \Gamma)^{2}}\right. \\
& \left.+\left(1+\frac{\Delta^{2}}{M_{n} M_{n+1}}\right) \frac{1}{\left(M_{n+1}+M_{n}\right)^{2}-(\omega+2 i \Gamma)^{2}}\right\},
\end{aligned}
$$

where $n_{F}(\epsilon)=1 /\left\{1+\exp \left[\left(\epsilon-\mu_{c}\right) /\left(k_{B} T\right)\right]\right\}$ is the Fermi-Dirac distribution, $v_{F}=10^{6} \mathrm{~m} / \mathrm{s}$ is the Fermi velocity, $M_{n}=\sqrt{\Delta^{2}+2 n \hbar v_{F}^{2}\left|e \mathbf{B}_{0}\right|}$ is the energy of the $n^{\text {th }}$ Landau level. $\Delta$ is the excitonic bandgap that can be assumed zero for low magnetic fields, leading to the following simplified equations:

$\sigma_{d}=-\frac{i e^{2}(\omega+i 2 \Gamma)}{\pi \hbar^{2}}\left[\frac{1}{(\omega+2 i \Gamma)^{2}} \int_{0}^{\infty} d \epsilon\left(\frac{\partial \mathrm{n}_{F}(\epsilon)}{\partial \epsilon}-\frac{\partial \mathrm{n}_{F}(-\epsilon)}{\partial \epsilon}\right) \epsilon-\int_{0}^{\infty} d \epsilon \frac{\mathrm{n}_{F}(-\epsilon)-\mathrm{n}_{F}(\epsilon)}{(\omega+2 i \Gamma)^{2}-4(\epsilon / \hbar)^{2}}\right]$,

and 


$$
\sigma_{0}=-\frac{e^{2} v_{F}^{2} e B_{0}}{\pi \hbar^{2}}\left[\frac{1}{(\omega+2 i \Gamma)^{2}} \int_{0}^{\infty} d \epsilon\left(\frac{\partial \mathrm{n}_{F}(\epsilon)}{\partial \epsilon}-\frac{\partial \mathrm{n}_{F}(-\epsilon)}{\partial \epsilon}\right)+\int_{0}^{\infty} d \epsilon \frac{1}{(\omega+2 i \Gamma)^{2}-4(\epsilon / \hbar)^{2}}\right]
$$

The off-diagonal term of conductivity in Eq. (3.31) vanishes by assuming no magnetic bias field $\mathbf{B}_{\mathbf{0}}=0$, while the diagonal term $\sigma_{d}$ in Eq. (3.30) are independent of the DC magnetic field.

Graphene's optical conductivity is divided into the intraband and interband parts, which correspond to free carrier absorption and transition from the valance band to the conduction band, respectively. In Eq. (3.30), the first term is due to intraband contributions and the second term is related to interband transitions. Eq. (3.32) below is the Drude-like analytical expression of the intraband transitions term [132, 140], while Eq. (3.33) below is the approximate analytical expression obtained for the interband term when $K_{B} T \ll\left|\mu_{c}\right|$ and $K_{B} T \ll \hbar \omega[141]$ :

$$
\begin{gathered}
\sigma_{d, \text { int } r a}=i \frac{e^{2} K_{B} T}{\pi \hbar^{2}(\omega+2 i \Gamma)}\left[\frac{\mu_{c}}{K_{B} T}+2 \ln \left(e^{-\frac{\mu_{c}}{k_{B} T}}+1\right)\right], \\
\sigma_{d, \text { inter }} \approx \frac{i e^{2}}{4 \pi \hbar} \ln \left(\frac{2\left|\mu_{c}\right|-(\omega+2 i \Gamma) \hbar}{2\left|\mu_{c}\right|+(\omega+2 i \Gamma) \hbar}\right) .
\end{gathered}
$$

The scattering rate $\Gamma$ has inverse relation to the relaxation time $\tau$ as $2 \Gamma=\tau^{-1}$. The relaxation time varies with the Fermi energy (chemical potential) for a fixed Fermi velocity of graphene as $\tau=\mu_{c} / e v_{F}^{2}$. It is worth mentioning that the high ambient temperature of thermal emitters invalidates the condition for the closed-form approximation in Eq. (3.33). In our study the general form in Eq. (3.30) is therefore evaluated numerically to obtain the exact value of the refractive index of graphene. However, Eq. (3.33) shows the relation of parameters to the interband contribution. For instance, this equation implies that $\sigma_{d \text {,inter }}$ is purely imaginary when $2\left|\mu_{c}\right|>\hbar \omega$ and $\Gamma \approx 0$, or complex when $2\left|\mu_{c}\right|<\hbar \omega$. 
The contributions of intraband and interband transitions in the optical conductivity significantly depend on the carrier density, so that each part has different strength at different frequency ranges. These contributions are also directly related to the chemical potential in graphene. Figure 3.3 describes the optical conductivity of graphene and its equivalent refractive index as a function of the chemical potential and the wavelength of incident light. It can be seen in Fig. 3.3(a) that, for undoped suspended graphene (i.e. there is no electric field to tune the chemical potential), the interband transitions are responsible for the $\sim 2.3 \%$ broadband absorption. The DC electric field bias tunes the chemical potential in graphene, adding either electrons or holes to the system. In this scenario, the interband transitions of electrons only occur for $2 E_{0}=\hbar \omega>2 E_{F}$, while other transitions are forbidden or blocked for $E_{0}<2 E_{F}$, as shown in Fig.

3.3(b). The absorption due to the interband transition is reduced by Pauli blocking, because the vacant states in conduction band are all occupied when the pumping light is intense enough for a specific relaxation process [108].

The intraband transitions are mainly responsible for the absorption in the far-infrared and also contribute, to some extent, to the mid-infrared optical response [142], as shown in Figs. 3.3(c) and 3.3(d). For short wavelengths in the visible range, graphene's optical conductivity is dominated by interband transitions [142]. The contribution of intraband transition decreases by increasing the chemical potential in the visible range, and has therefore no significant effect on graphene's optical conductivity at these wavelengths.

Finally, the dielectric permittivity of monolayer graphene $\varepsilon_{G}$ at optical frequencies is given by $[59,61]$

$$
\varepsilon_{G}=\frac{i \sigma_{d}}{\omega \varepsilon_{0} t_{G}}
$$


where $\sigma_{d}$ is the conductivity of graphene, $t_{G}$ is the thickness of a single graphene layer, $\omega$ is the angular frequency, and $\varepsilon_{0}$ is the free-space electric permittivity. Figs. 3.3(e) and 3.3(f) show the real and imaginary parts of graphene's refractive index as a function of the chemical potential in graphene. It can be observed that in the visible range, the real and imaginary parts of graphene's refractive index are nearly independent of the chemical potential.
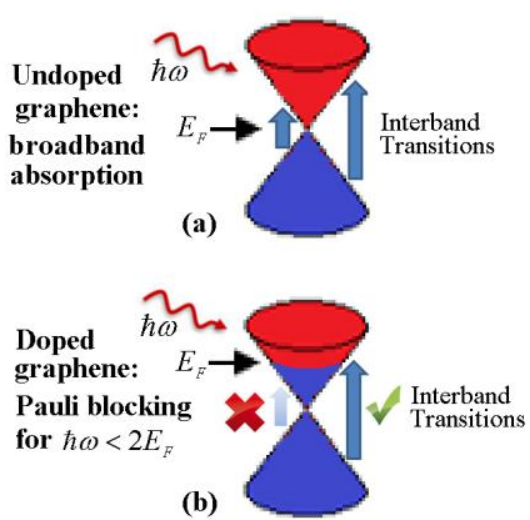

(b)

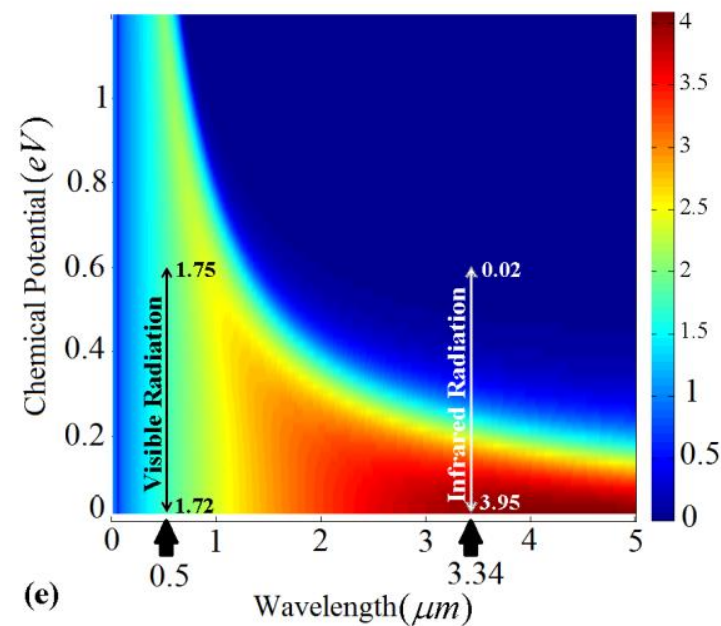

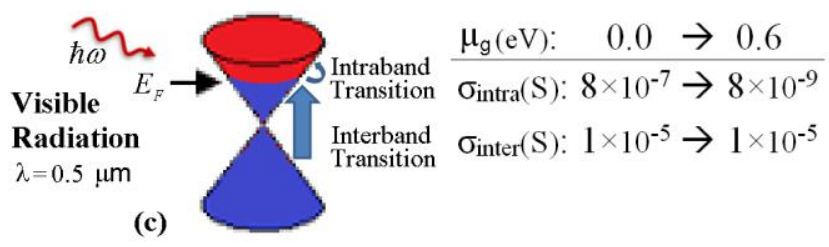

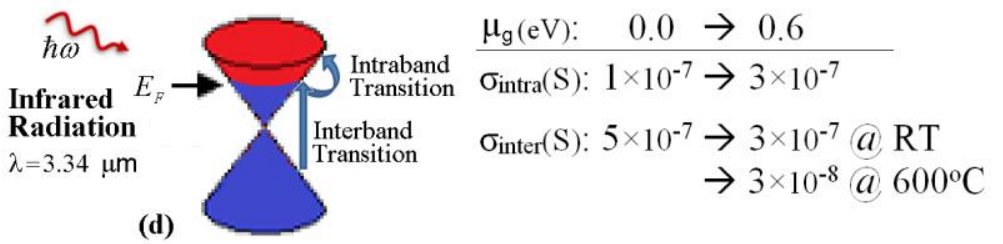

(d)

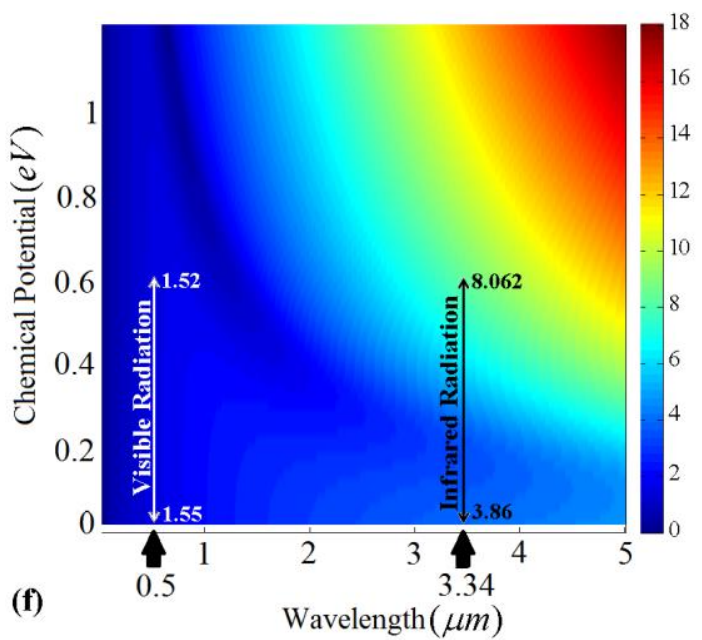

Figure 3.3. Sketch of graphene's dispersion for different chemical potentials. (a) For undoped graphene, all the interband transitions are allowed; (b) For doped graphene, interband transitions are forbidden for $\hbar \omega<2 E_{F}$ by the Pauli blocking; (c) For visible light, the optical conductivity due to interband transitions is dominant; (d) For infrared radiation, the interband and intraband transitions have comparable contributions to the optical conductivity of graphene; (e) The real part and (f) imaginary parts of the refractive index obtained by the Kubo formalism, as well as the equivalent changes in their values for visible and infrared radiation for chemical potential of $\mu_{c}=0.0 \mathrm{eV}$ and $\mu_{c}=$ $0.6 \mathrm{eV}$. 
For longer wavelengths in infrared range, the Drude-like part in Eq. (3.32) is increasing as the wavelength becomes longer, while the interband transition contribution significantly decreases behaving as a step-like function with threshold $2\left|E_{F}\right|$ (small value when the photon energy is below $2\left|E_{F}\right|$, and large value when the photon energy is above $2\left|E_{F}\right|$ ). As such, the intraband transition contribution becomes comparable with the interband transition contribution, as shown in Fig. 3.3(d). The control over intraband transitions can be obtained by tuning of the chemical potential in graphene, resulting in graphene applications in the infrared and THz ranges [102, 143, 144]. As such, graphene has been shown to be a good candidate for plasmonics and metamaterials in the mid-infrared $[145,146]$ and $\mathrm{THz}$ ranges $[144,147,148]$. For infrared radiation at high ambient temperature of thermal emitter (e.g. $873 \mathrm{~K}$ in this thesis), the results show an order of magnitude decrease in the contribution of interband transitions, leading to larger contribution of intraband transitions to the total optical conductivity of graphene. 


\section{CHAPTER 4 \\ APERIODIC GRAPHENE BASED MULTILAYER STRUCTURE AS A SELECTIVE, TUNABLE, AND SWITCHABLE THERMAL EMITTER}

\subsection{Introduction}

The thermal emittance of both bulk materials and multilayer structures has been widely investigated over recent years. The thermal radiation from bulk materials is characterized by incoherent, isotropic, and broadband radiation spectra that vary depending on the properties of the selected material and its temperature. The thermal radiation spectra can be drastically altered by utilizing textured surfaces [149] and multilayer structures [150]. Highly directional and quasicoherent thermal radiation sources, have many important applications as antennae [13], lasers [34, $151]$, and in photovoltaics $[152,153]$.

In this chapter, aperiodic multilayer structures are proposed as narrowband, tunable, and switchable thermal infrared emitters, tailoring the radiation spectra of bulk materials [152]. Using a genetic global optimization algorithm (Section 3.4) coupled to a transfer matrix code (Section 3.3), the layer thicknesses and materials are optimized to maximize the absorptance at a single wavelength, leading to a narrowband thermal infrared emitter. The effect of chemical potential of graphene layers on the tunability and switchability of this narrowband emission from the proposed aperiodic multilayer structure is also investigated.

\subsection{Structure of graphene-based thermal emitter}

Figure 4.1(a) shows the schematic of the proposed structures composed of alternating layers of graphene and hBN insulator, which are sandwiched between two thick silicon carbide (SiC) layers. In many cases, a multilayer structure may provide spectra-altering properties similar to that of more complex and harder-to-fabricate two- or three-dimensional structures [152]. hBN is an insulating layer with atomic thickness similar to graphene (see Fig. 4.1(b)), thereby accurate control of the spacing between the graphene layers can be achieved in the proposed aperiodic 
structures [154]. The thicknesses of $\mathrm{SiC}$ layers and number of $\mathrm{hBN}$ layers are found by applying the genetic algorithm to achieve a structure with perfect absorptance equal to unity. The density of charge carriers associated with the chemical potential in graphene layers can be controlled by applying a DC bias field perpendicular to the graphene/hBN surfaces [155].

A semi-infinite tungsten (W) layer is used as the substrate. Since the tungsten substrate is taken to be semi-infinite, the transmittance is identically zero, so that:

$$
A_{T E / T M}(\lambda)=1-R_{T E / T M}(\lambda),
$$

where $A_{T E / T M}(\lambda)$ is the TE/TM absorptance, $R_{\text {TE/TM }}(\lambda)$ is the TE/TM reflectance, and $\lambda$ is the wavelength. The calculated absorptance can be equated to emittance because of Kirchhoff's second law and conservation of energy under thermal equilibrium, as explained in Chapter 3. The Kubo formula (Section 3.5) [132] is used to extract the optical conductivity and consequently the refractive index of graphene. For other materials such as $\mathrm{hBN}, \mathrm{SiC}$ and $\mathrm{W}$, the wavelengthdependent indices of refraction (both real and imaginary parts) are obtained from experimental data [156-158]. The effect of layer thickness variations due to thermal expansion on emittance/absorptance can be neglected. In addition, all materials used in the structure can tolerate high temperatures due to their high melting points [159].

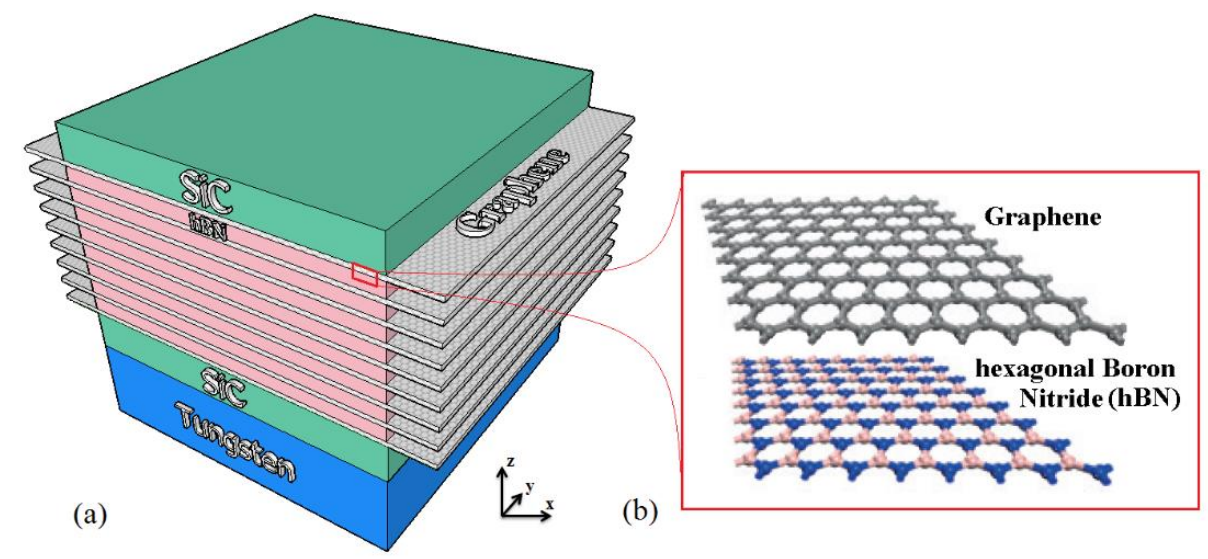

Figure 4.1: (a) Structure of the proposed thermal emitter. (b) The lattice structure of graphene and hBN buffer layer. 


\subsection{Optimized structure of narrowband thermal emitter}

Using the transfer matrix method and the genetic algorithm as outlined in Chapter 3, the aperiodic multilayer structure is optimized to produce narrowband infrared thermal emission for both TE and TM polarizations. Thermal emission is considered at ambient temperature of $873 \mathrm{~K}$ corresponding to the maximum emission of blackbody at infrared range with the peak at $\lambda=3340$ $\mathrm{nm}$. This is the wavelength at which the genetic algorithm is applied to find the optimized structures with maximum absorptance. To seek the optimum structure, the chemical potentials of graphene layers are set equal to zero $\left(\mu_{c}=0.0 \mathrm{eV}\right)$, i.e. the chemical potentials are located at the Dirac point of graphene dispersion.

Figure 4.2(a) shows the aperiodic structure including the graphene layers (black lines) and layers of hBN insulator. Utilizing the genetic optimization algorithm, each layer's thickness is selected independently so that the structure is completely aperiodic. Finally, utilizing the transfer matrix method [11], the absorptance, which is equal to the emittance, of the graphene based structure is calculated.

Figure 4.2(b) shows $\bar{\mu}(\lambda)$ [Eq. (3.2)] as a function of wavelength for the structure in Fig. 4.2(a). We observe that the optimized structure exhibits almost perfect emittance of $\sim 100 \%$ at $\lambda=3.34 \mu \mathrm{m}$. The narrowband perfect emittance is accomplished through interaction with the layered nanophotonic structure so that the bandwidth of $2.7 \mu \mathrm{m}$ at $\mathrm{T}=873 \mathrm{~K}$ reduces to $0.63 \mu \mathrm{m}$, showing more than 4 times narrower bandwidth compared to the blackbody radiation curve. As shown in Fig. 3.1, the blackbody thermal emission is broadband, especially for lower ambient temperatures. The graphene-based heterostructure is an ultrathin nanophotonic structure that enables narrowband infrared emittance. 


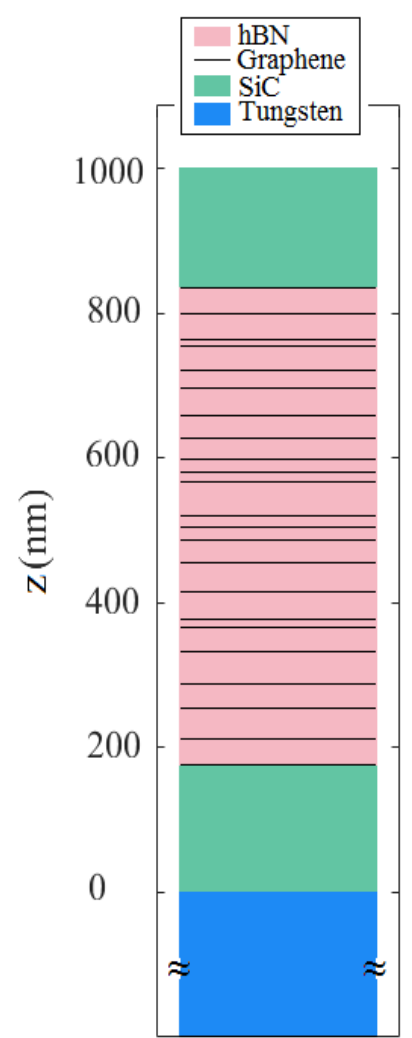

(a)

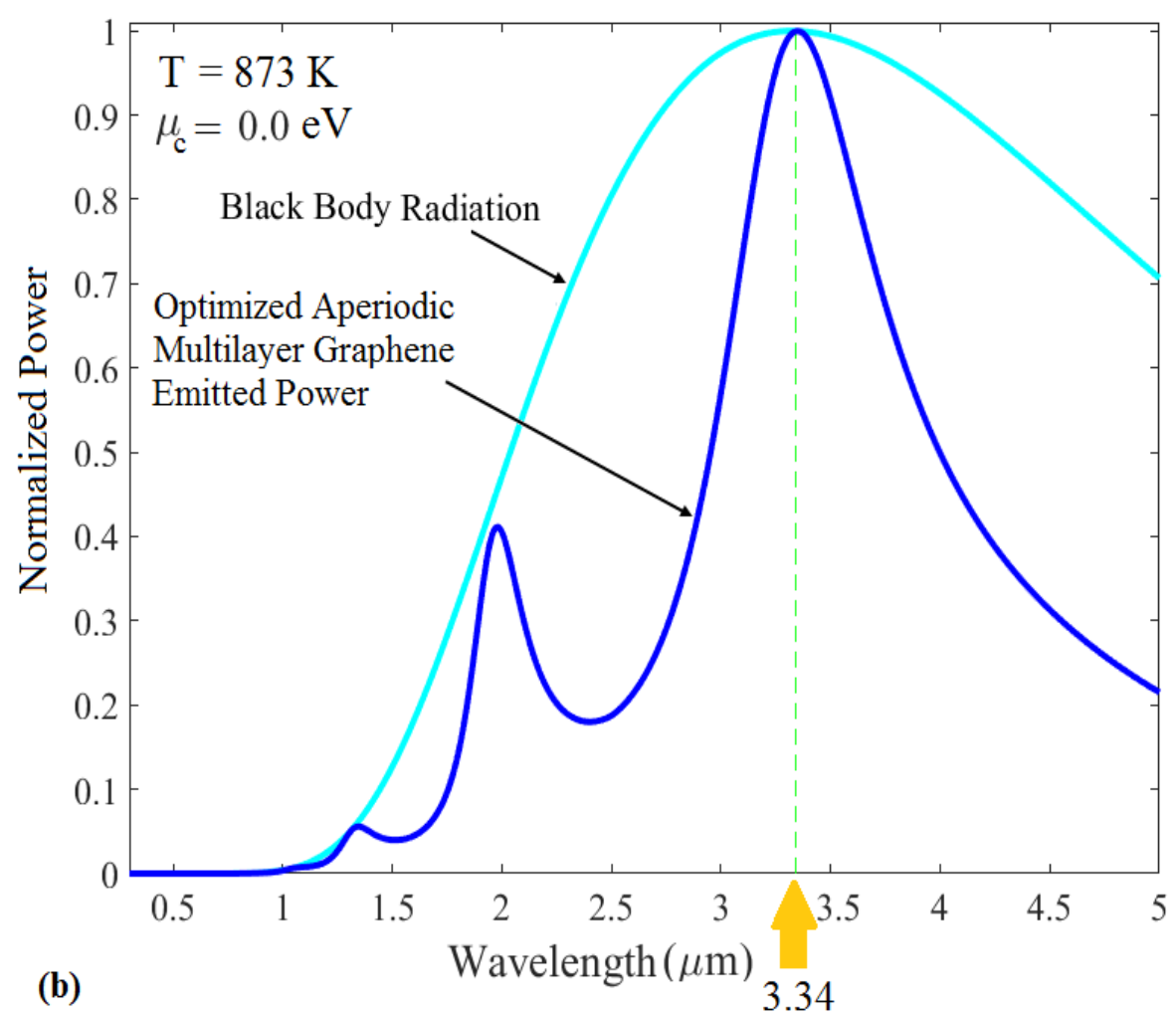

Figure 4.2: (a) Optimized structure with 23layers of graphene; (b) Normalized power radiated per unit area and unit wavelength by the structure in the normal directon. Note: the layer thicknesses for the structure in (a) are given in appendix A.

The optimized thickness of the smallest hBN layer is found to be $d=8.9 \mathrm{~nm}$ corresponding

to $27 \mathrm{hBN}$ layers. In Fig. 4.3, the effect of this layer thickness on the emittance spectra of the optimized structure is investigated. The thickness is changed to $5 \times d, 10 \times d$, and $20 \times d$, while the thicknesses of other layers are constant. This study can indicate the sensitivity of the optimized structure to fabrication process variations. Figure 4.3(b) shows the contribution of graphene layers and tungsten to the total power emitted from the proposed structure for different thicknesses. The $\mathrm{SiC}$ layers are not shown in the figure because it has less than $2 \%$ contribution to the total power regardless of the thickness of the smallest hBN layer. At the optimized wavelength $\lambda=3.34 \mu \mathrm{m}$ (dotted line), the tungsten and graphene layers contribute $\sim 46 \%$ and $\sim 52 \%$, respectively, to the 
total power emitted from the optimized structure. The increase in the thickness of the thinnest hBN layer leads to increase in the reflectance so that, for the modified hBN thickness of $20 \times d$, the contributions of the tungsten and graphene layers are dramatically decreased to $\sim 20 \%$ and $\sim 25 \%$, respectively. It can be observed that, by increasing the thickness of the narrowest hBN layer from the value that is obtained from the optimization process, the peak of radiated power shifts to longer wavelengths and also decreases.

The electric field in the structure can also be calculated for both TE and TM polarizations using the transfer matrix method [11] as explained in Section 3.3. Figure 4.4(a) shows the profile of the electric field amplitude normalized with respect to the field amplitude of the incident plane wave for the optimized structure in Fig. 4.2(a). The structure is excited by a normally incident plane wave at $\lambda=3340 \mathrm{~nm}$, the wavelength at which the structures are optimized. The effect of varying the chemical potential of graphene can be clearly observed in the electric field profiles. For $\mu_{c}=0.0 \mathrm{eV}$, at which the structure is optimized to achieve maximum absorptance, the electric field amplitude is almost flat in air. This suggests that the reflectance of the structure is almost zero, and the absorptance is therefore almost unity.

Figure 4.4(b) shows the contribution of each graphene layer to the total emittance for $\mu_{c}=$ $0.0 \mathrm{eV}, 0.4 \mathrm{eV}$, and $1.0 \mathrm{eV}$. It is obvious that the contribution of graphene layers to the total power emitted from the proposed structure drastically decreases by increasing the chemical potential. The relative contribution to absorptance/emittance for each graphene layer follows the square of the field amplitude in the structure and the real and imaginary parts of the refractive index, which can be manipulated by changing the chemical potential of graphene [Eq. (3.25)]. The change in the properties of the thermal emittance, induced by changing the chemical potential of the graphene layers enables an electrically controllable thermal emitter, as explained in the next chapter. 


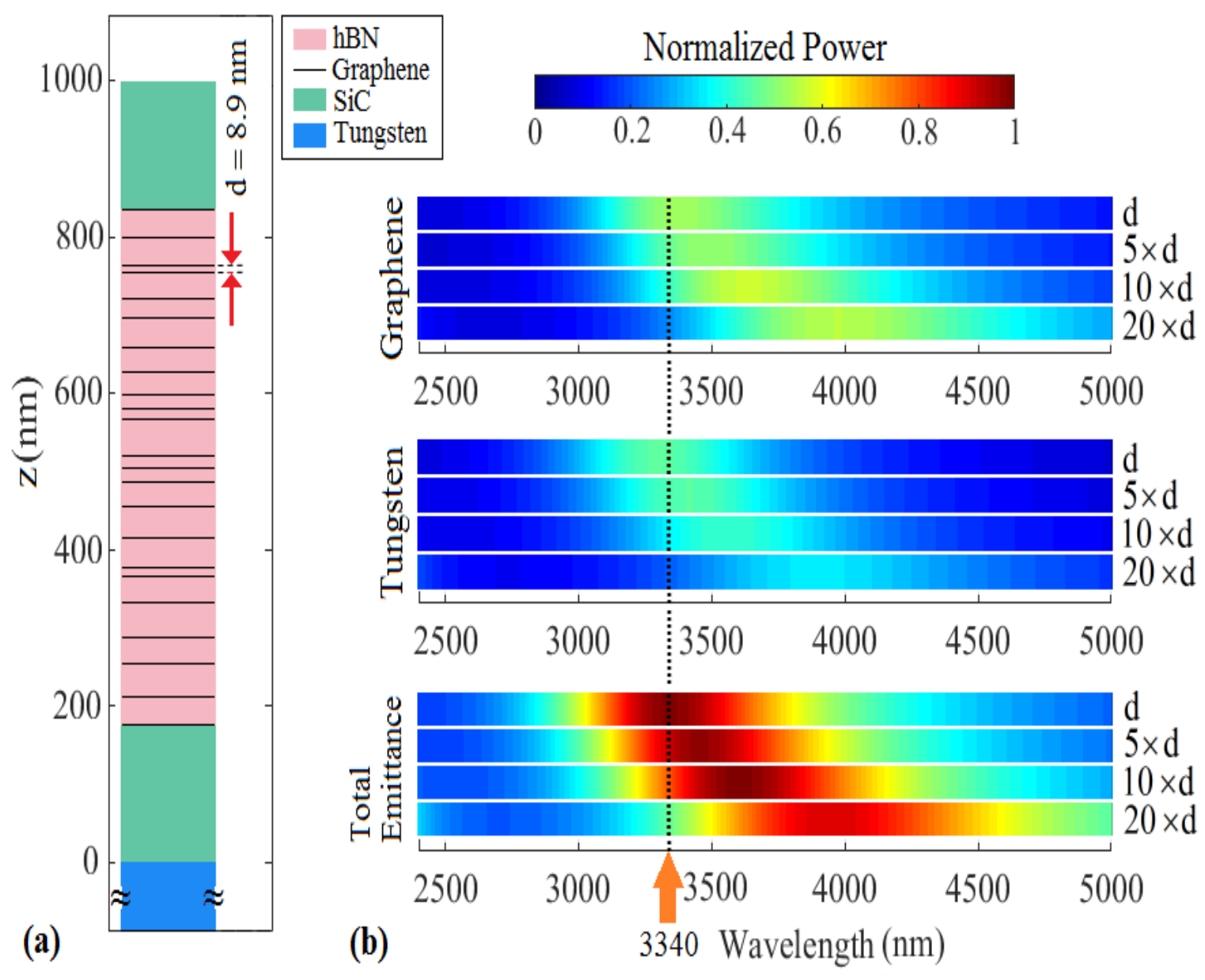

Figure 4.3: (a) Optimized structure with 23 layers of graphene including the location of thinnest hBN layer. (b) Effect of changing the optimized thickness of the thinnest hBN layer on the emittance spectra, as well as on the contribution of graphene layers and tungsten to the total emittance. 

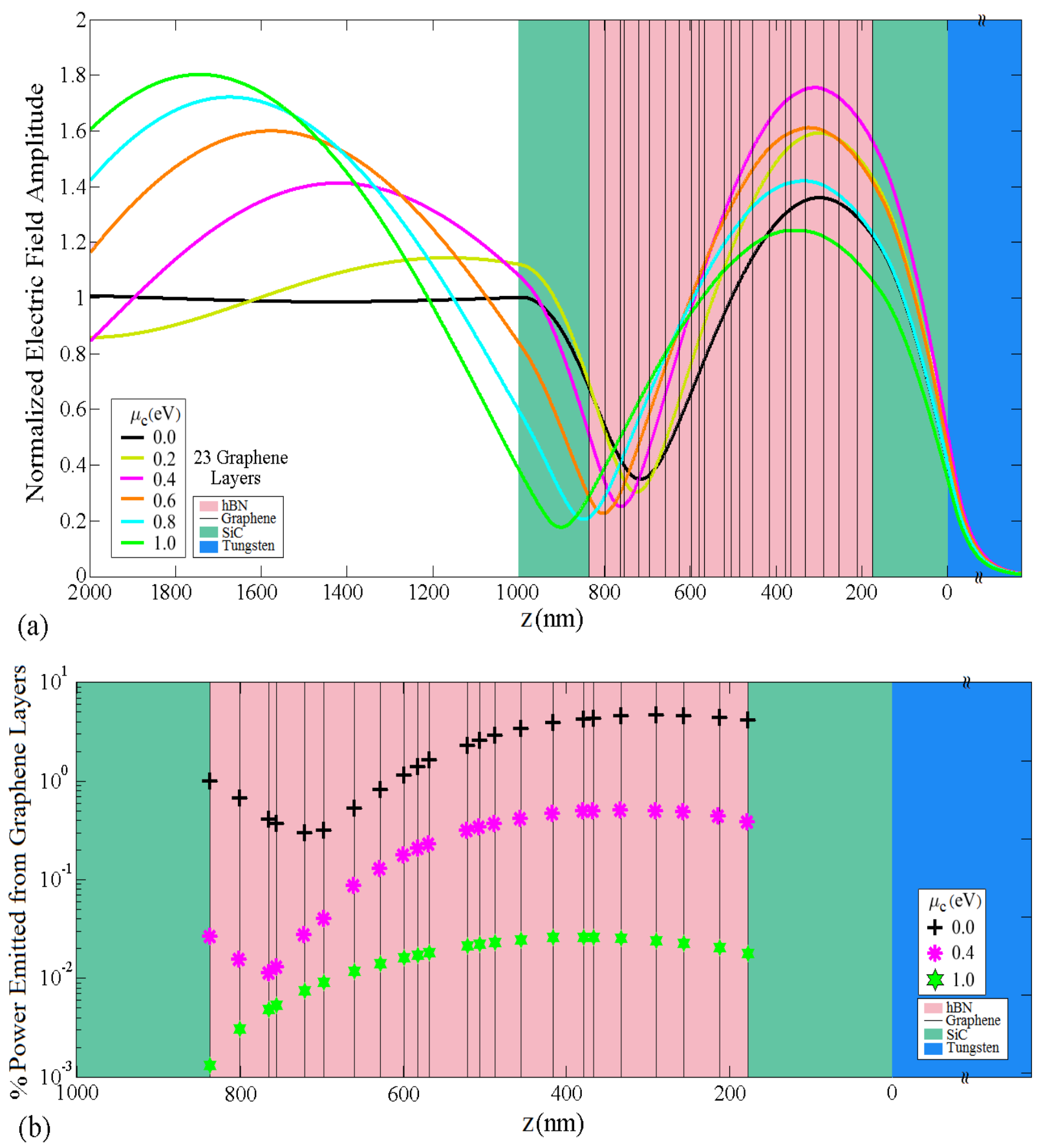

Figure 4.4: (a) Electric field amplitude normalized with respect to the field amplitude of the incident plane wave for the optimized structure with 23 graphene layers; (b) Contribution of each graphene layer to the total power emitted for $\mu_{c}=0.0 \mathrm{eV}, 0.4 \mathrm{eV}$ and $1.0 \mathrm{eV}$. 


\subsection{Tunability and switchability of graphene-based thermal emitter}

Figure 4.5 shows the partial absorptance and the corresponding reflectance as a function of wavelength for three different chemical potentials in graphene, $\mu_{c}=0.0 \mathrm{eV}, 0.4 \mathrm{eV}$, and $1.0 \mathrm{eV}$. At the chemical potential of $\mu_{c}=0.0 \mathrm{eV}$, it is obvious that the reflectance is zero at the wavelength of $3.34 \mu \mathrm{m}$ at which the structure is optimized, and perfect absorptance can be achieved as indicated in Eq. (4.1). By increasing the chemical potential of graphene layers, the reflectance is increased and the corresponding absorptance on graphene layers is decreased at the wavelength at which the structure is optimized. As such, at $\mu_{c}=1.0 \mathrm{eV}$ tungsten is almost the only layer that contributes to the total absorption at the wavelength of $3.34 \mu \mathrm{m}$. At this wavelength, the absorptance is $1.0,0.97,0.82,0.64,0.48$, and 0.35 for chemical potential of $\mu_{c}=0.0 \mathrm{eV}, 0.2 \mathrm{eV}$, $0.4 \mathrm{eV}, 0.6 \mathrm{eV}, 0.8 \mathrm{eV}$, and $1.0 \mathrm{eV}$, respectively. This shows the possibility of switching, i.e. turning off, the absorptance of the proposed structure.

Figure 4.6 shows the effect of the chemical potential on the absorptance of the optimized structure. It can be observed that the maximum of the absorptance is close to unity but shifts to shorter wavelengths, as the chemical potential is varied. This shift is associated with the change of the chemical potential of graphene. In other words, the increase in the chemical potential reduces the absorption in graphene layers, but other layers and especially tungsten, increase their contribution to the total absorption, so that the maximum absorption shifts to shorter wavelengths. This indicates that the proposed graphene-based aperiodic structure can be electrically controlled, enabling a tunable thermal emitter. In the next chapter, we shed more light on the switchability and tunability of the proposed graphene-based thermal emitters. 

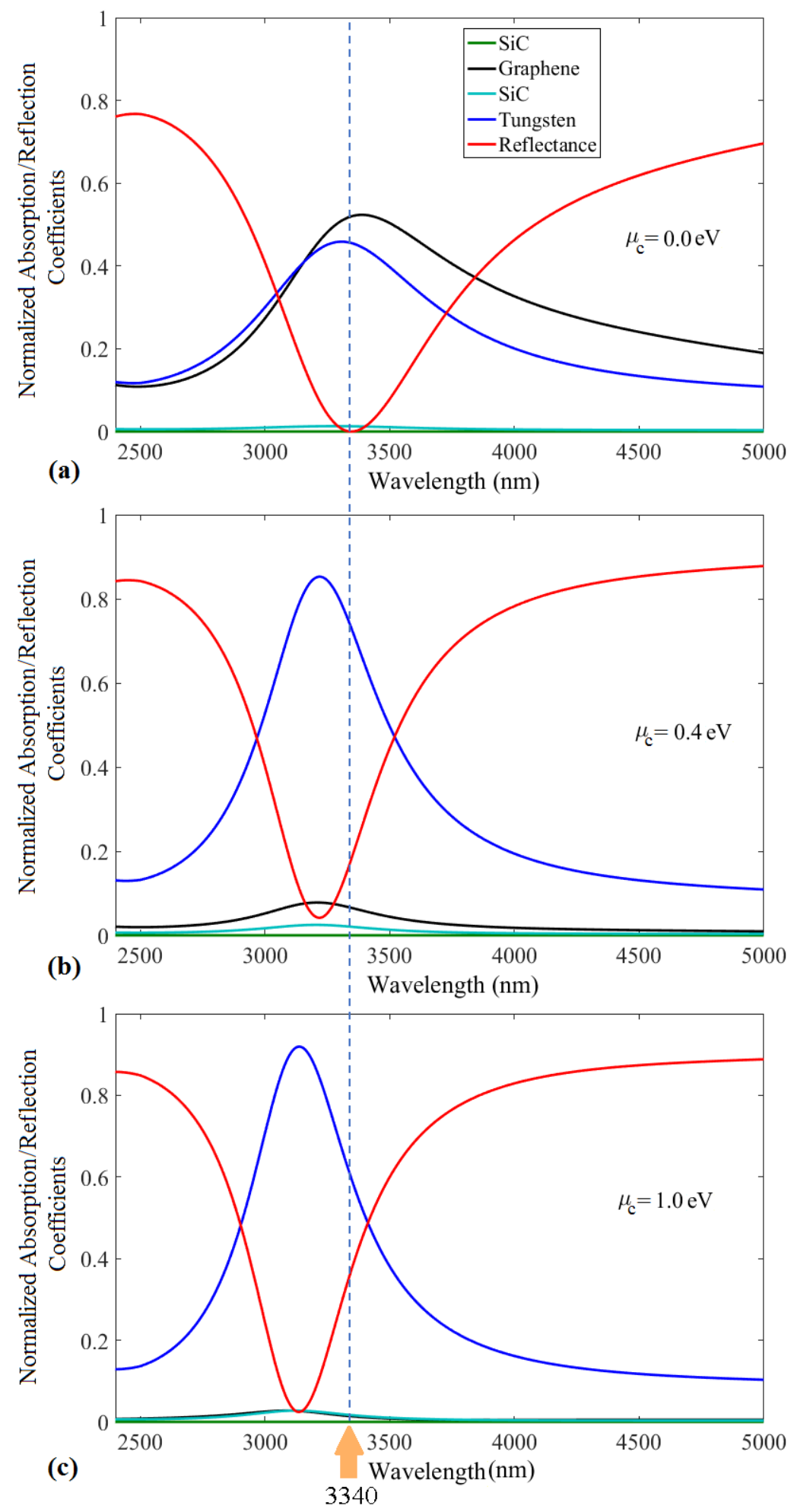

Figure 4.5: Effect of changing the chemical potential in graphene, i.e. $\mu_{c}=0.0 \mathrm{eV}, 0.4 \mathrm{eV}$, and 1.0 $\mathrm{eV}$, on the partial absorptance and the reflectance. 


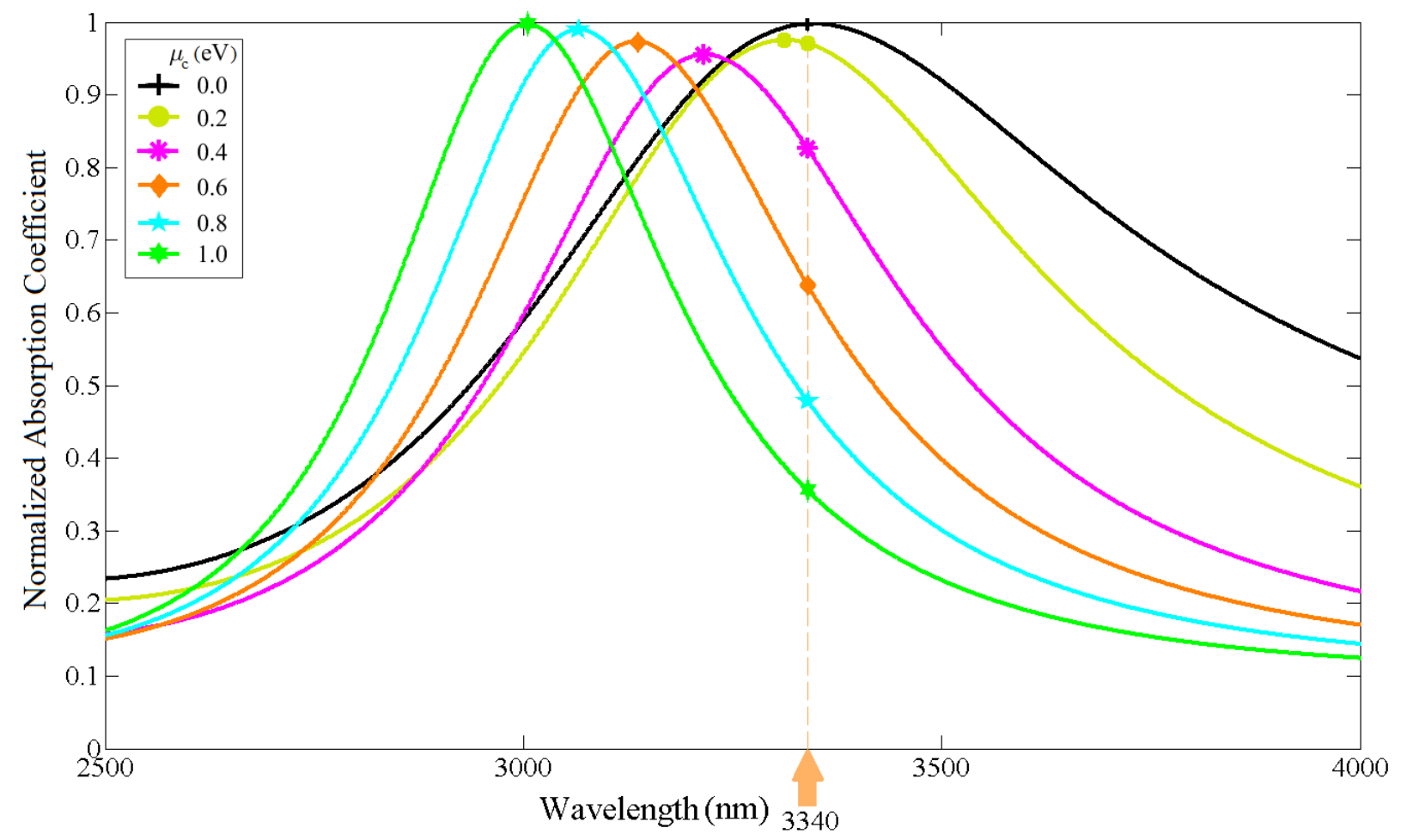

Figure 4.6: Absorptance of the optimized structure in Fig. 4.2(a) for different chemical potentials, $\mu_{c}=0.0 \mathrm{eV}, 0.2 \mathrm{eV}, 0.4 \mathrm{eV}, 0.6 \mathrm{eV}, 0.8 \mathrm{eV}$, and $1.0 \mathrm{eV}$. 


\section{CHAPTER 5 \\ COMPARISON OF APERIODIC GRAPHENE BASED MULTILAYER THERMAL EMITTERS WITH DIFFERENT NUMBER OF GRAPHENE LAYERS}

\subsection{Introduction}

It has been demonstrated that multilayer structures modify the absorption spectra of bulk materials. This is important for many applications involving metallic gratings [9, 155], metallic and semiconductor photonic crystals [160-162], periodic grooves [163], and nano-antennae [13]. Unusual absorption characteristics in the infrared wavelength range are important for many applications such as photovoltaics [164, 165], control of thermal radiation [12, 166], photodetectors [167, 168] and chemical sensing [161, 169]. As in [21, 170-173], our effort here is to design photonic nanostructures with near perfect and narrowband, i.e. selective, thermal emission [21, 170-173]. However, our graphene-based aperiodic multilayer structures not only enable narrowband thermal emission at mid-infrared wavelengths, but also provide tunable and switchable thermal emitters. In this chapter, several aperiodic multilayer structures with different number of graphene layers are optimized using the genetic optimization algorithm to determine the best dimensions of thermal emitters for the sake of improved selectivity, tunability, and switchability.

\subsection{Optimized graphene based thermal emitters}

Figure 5.1 shows five aperiodic thermal emitters with 8, 13, 23, 28, and 32 layers of graphene. By considering these five structures, the effect of the number of graphene layers on bandwidth, tunability, and switchability is studied. For a fair comparison, the overall thicknesses of these structures are kept approximately equal to $1 \mu \mathrm{m}$ to minimize the potential effect of the overall thicknesses. The thicknesses of all layers in these structures are reported in appendix A. 


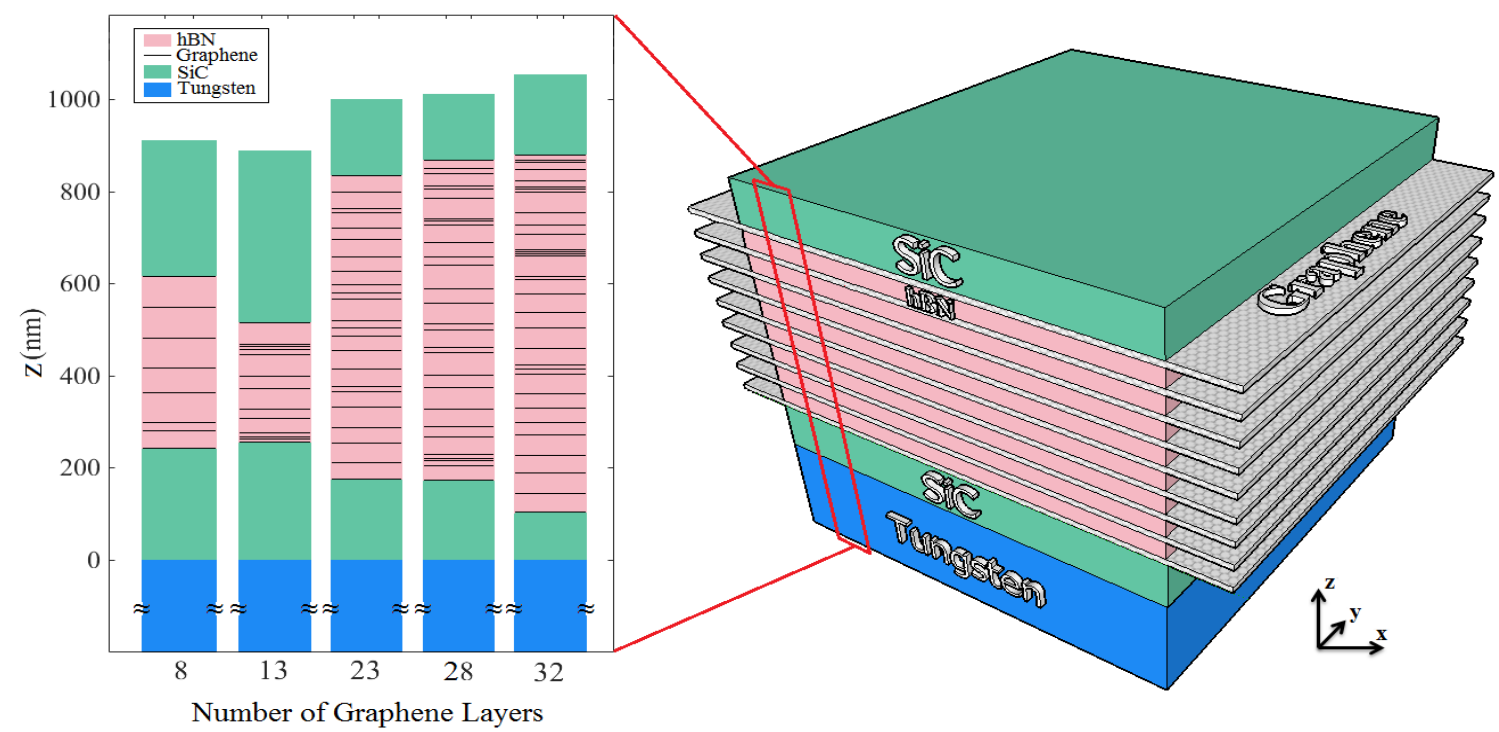

Figure 5.1: Aperiodic multilayer structures with 8, 13, 23, 28, and 32 layers of graphene optimized using the genetic algorithm. Note: the layer thicknesses for each structure are given in appendix A.

Figure 5.2 shows the normalized power radiated per unit area and unit wavelength by the structures in the normal direction as a function of wavelength. It can be observed that all five structures exhibit perfect emittance at $\lambda=3.34 \mu \mathrm{m}$, which corresponds to the maximum thermal emission of a blackbody at $\mathrm{T}=873 \mathrm{~K}$. Despite the broadband spectra of blackbody radiation, the graphene-based nanostructures enable narrowband infrared emittance. Interestingly, the increase in the number of graphene layers does not result in narrower thermal emission. Thus, the bandwidth of the power emitted from the structure with the smallest number of graphene layers, i.e. 8 graphene layers, is narrower than the one of the structure with the largest number of graphene layers, i.e. 32 graphene layers. However, this increase in the number of graphene layers decreases the strength of undesired power emitted at shorter wavelengths. Even though the structure with 8 graphene layers shows better selectivity of thermal power emission than the structure with 32 graphene layers at zero bias $\left(\mu_{c}=0.0 \mathrm{eV}\right)$, the increase in the number of graphene layers enhances the effect of varying the chemical potential of graphene layers, promoting larger range of tunability and stronger switchability as discussed in the next section. 


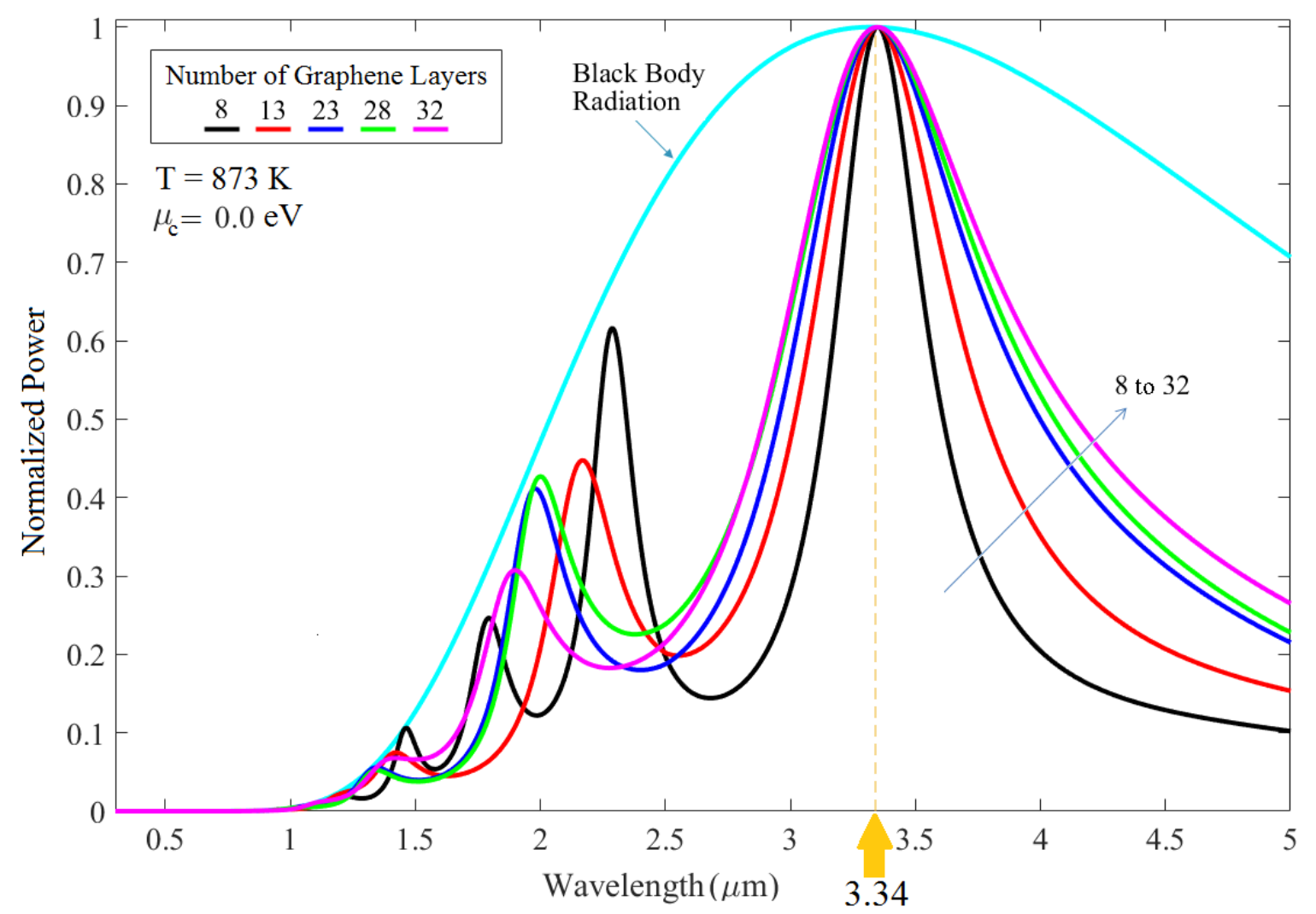

Figure 5.2: Normalized power radiated per unit area and unit wavelength in the normal direction by the structures of Fig. 5.1 as a function of wavelength.

\subsection{Effect of chemical potential on selectivity, tunability, and switchability}

After obtaining the optimized structure for $\mu_{c}=0.0 \mathrm{eV}$, the carrier density in graphene is increased by applying a positive DC bias, that corresponds to tuning the chemical potential to larger values. The increase in the chemical potential leads to modification of the optical properties of the structures $[63,174]$, as explained in Section 3.5. Figure 5.3(a) shows the thermal power emitted from bulk tungsten at $\mathrm{T}=873 \mathrm{~K}$. Figures 5.3(b-f) depict the effect of the increase in the chemical potential on the normalized power emitted from the five optimized structures with 8,13 , 23, 28, and 32 layers of graphene.

For the optimized thermal emitter with 8 graphene layers in Fig. 5.3(b), it can be observed that the increase in the chemical potential results in a spectral shift towards shorter wavelengths 


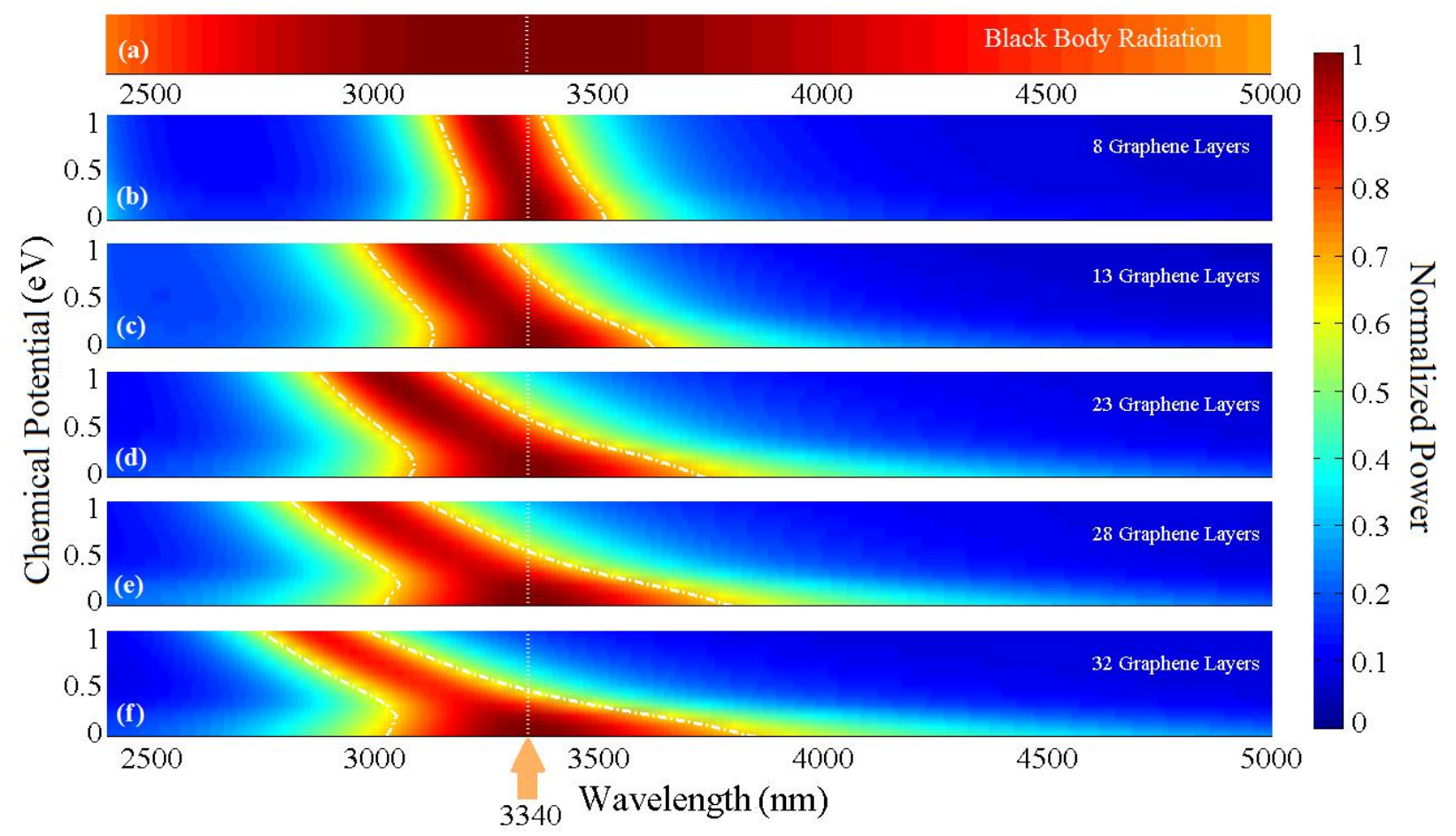

Figure 5.3: (a) Normalized power emitted per unit area and unit wavelength in the normal direction from bulk tungsten at $\mathrm{T}=873 \mathrm{~K}$. Normalized power emitted, per unit area and unit wavelength in the normal direction as a function of wavelength and chemical potential at $\mathrm{T}=873 \mathrm{~K}$ for the five optimized structures with (b) 8 , (c) 13, (d) 23, (e) 28, and (f) 32 layers of graphene. Note: the dotted vertical line shows the wavelength of $\lambda=3.34$ $\mu \mathrm{m}$ at which the structures are optimized and the dash-dotted lines correspond to $0.7 \times \max [\bar{\mu}(\lambda)]$, which is used to define the bandwidth of the emission.

and in narrower thermal emission. Comparing these results to the ones for the other optimized structures with larger number of graphene layers in Figs. 5.3(c-f), one can notice the more pronounced effect of chemical potential variation on the peak emission wavelength and the emission bandwidth as the number of graphene layers is increased.

The switchability can be inferred from the figure by looking at the dotted line that corresponds to the wavelength at which the structures are optimized. It can be observed that, for the optimized structure with 8 graphene layers, changing the chemical potential from $0.0 \mathrm{eV}$ to 1.0 $\mathrm{eV}$ does not result in significant change in the normalized power emitted from the structure. However, the normalized power emitted from the optimized structure with 32 graphene layers can 
be almost completely eliminated by increasing the chemical potential in this range, so that perfect emittance of unity at $\mu_{c}=0.0 \mathrm{eV}$ can be switched to zero by setting $\mu_{c}$ equal to $1.0 \mathrm{eV}$.

In the rest of this chapter, the selectivity, tunability, and switchability of the thermal emittance is studied for the optimized aperiodic multilayer structures with $8,13,23,28$, and 32 graphene layers by varying the chemical potential from $0.0 \mathrm{eV}$ to $1.0 \mathrm{eV}$.

While blackbody thermal emission is broadband, narrowband thermal emission can be achieved using the optimized nanophotonic structures. Figure 5.4 shows the effect of changing the chemical potential on the bandwidth of the thermal power emitted from the optimized structures with different numbers of graphene layers, i.e. the selectivity of the structures. The bandwidth $\Delta \lambda$ is measured at the wavelengths at which the normalized power emitted becomes $0.7 \times \max [\bar{\mu}(\lambda)]$. It can be observed that for all the optimized structures the selectivity of thermal emittance in wavelength becomes stronger by increasing the chemical potential. At $\mu_{c}=0.0 \mathrm{eV}$, the structure with 8 graphene layers has the power spectrum with the narrowest bandwidth, $\Delta \lambda=315 \mathrm{~nm}$, i.e. better selectivity in wavelength, while the power emitted from the structure with 32 graphene layers has about three times broader bandwidth. However, the larger number of graphene layers in the structure provides stronger control of the bandwidth by increasing the chemical potential. The power emitted from the structure with 32 graphene layers becomes three times narrower, changing from $\Delta \lambda=874 \mathrm{~nm}$ to $286 \mathrm{~nm}$, by increasing the chemical potential from $0.0 \mathrm{eV}$ to $1.0 \mathrm{eV}$, while the bandwidth of the 8 layer graphene structure only changes from $\Delta \lambda=315 \mathrm{~nm}$ to $234 \mathrm{~nm}$ for the same change in the chemical potential. As such, the nanophotonic structure with 32 layers of graphene enables stronger selectivity for thermal emission, which is electrically controllable by tuning the chemical potential in graphene layers. 


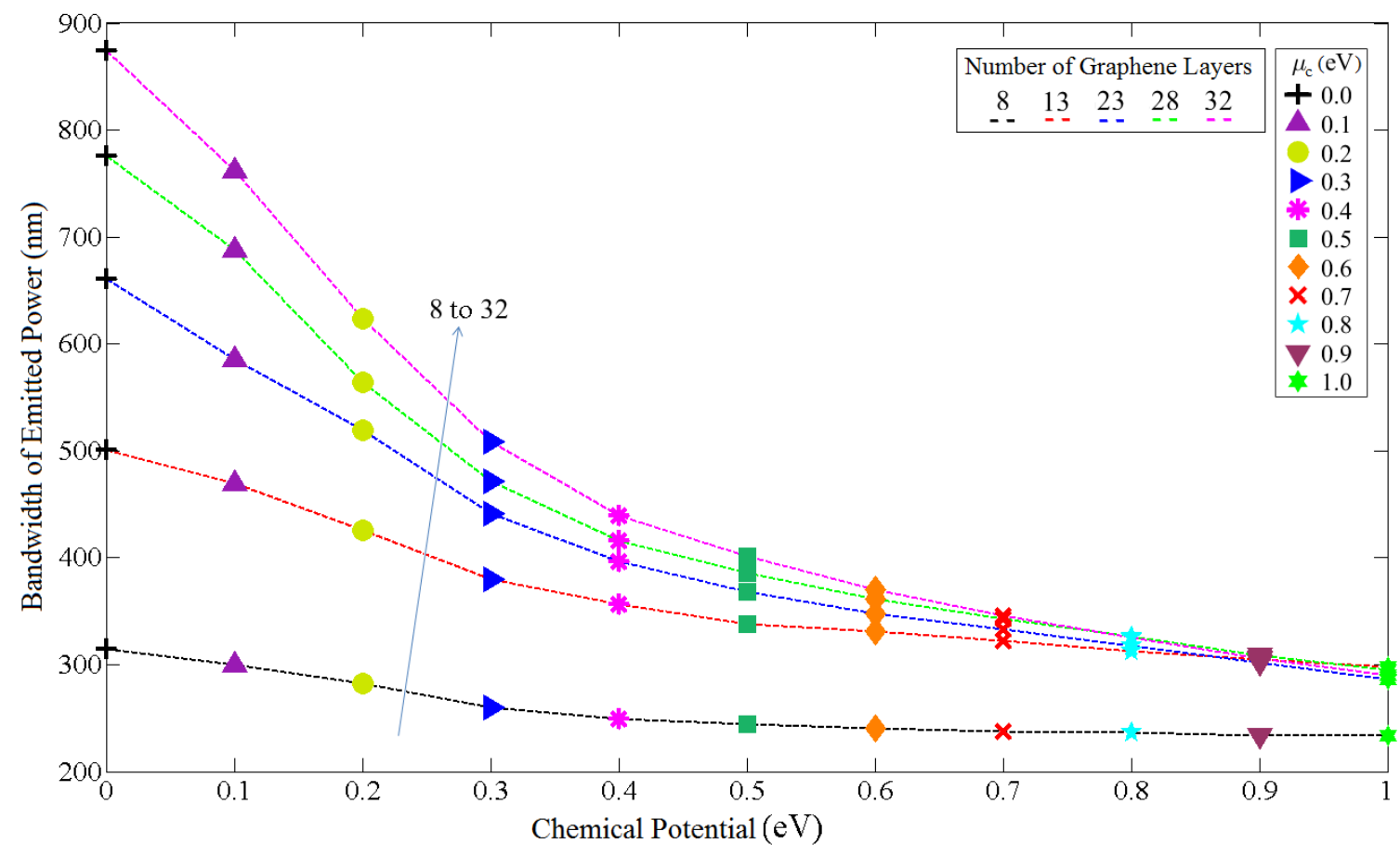

Figure 5.4: Bandwidth $\Delta \lambda$, i.e. selectivity, of the thermal power emitted from the optimized structures with different numbers of graphene layers versus chemical potential. The bandwidth is measured at the wavelengths at which the normalized power emitted becomes $0.7 \times \max [\bar{\mu}(\lambda)]$.

Figure 5.5 shows the effect of changing the chemical potential on the tunability of the thermal power emitted from the optimized structures with different numbers of graphene layers. It can be observed that the normalized power emitted from all the structures can be shifted to lower wavelengths by increasing the chemical potential in graphene. The range of tunability is increased by increasing the number of graphene layers in the aperiodic multilayer structures. For instance, the shift of the peak emission for the structure with 32 graphene layers is $\sim 3.5$ times larger than the one for the structure with 8 graphene layers, changing from $\lambda=3.34 \mu \mathrm{m}$ to $2.85 \mu \mathrm{m}$ by increasing $\mu_{c}=0.0 \mathrm{eV}$ to $1.0 \mathrm{eV}$. However, the peak normalized power emitted from the structures deviates from the one for perfect emitters, especially in the middle of the chemical potential range. Overall, the aperiodic multilayer structure enables a tunable thermal emitter that can be electrically controlled by changing the chemical potential in graphene layers. 


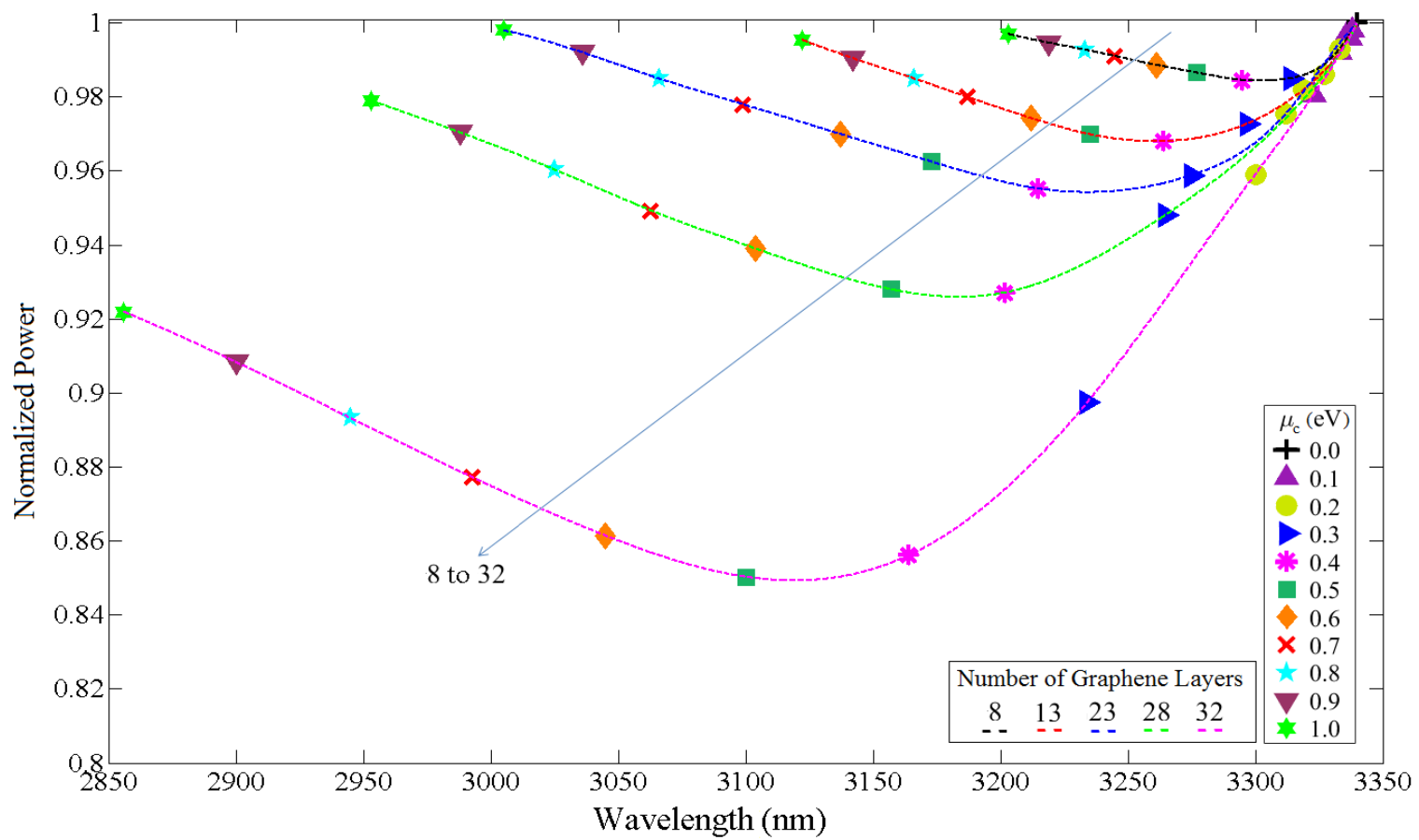

Figure 5.5: Tunability of the peak normalized power emitted per unit area and unit wavelength in the normal direction for the optimized structures with different number of graphene layers when the chemical potential is varied.

Figure 5.6 shows the effect of changing the chemical potential on the thermal power emitted at $\lambda=3.34 \mu \mathrm{m}$ for the optimized structures with different numbers of graphene layers. It can be observed that the normalized power emitted for all the optimized structures significantly decreases by increasing the chemical potential in graphene. For instance, by increasing the chemical potential form $0.0 \mathrm{eV}$ to $1.0 \mathrm{eV}$, the normalized power emitted from the structure with 8 graphene layers decreases by $\sim 25 \%$, changing from perfect value of unity to $\sim 0.75$. The range of change in thermal emission increases by increasing the number of graphene layers in the aperiodic multilayer structures, so that for the structure with 32 graphene layers the normalized emitted power at $\mu=1.0 \mathrm{eV}$ decreases by $\sim 83 \%$, which is about 4.5 times larger decrease than for the structure with 8 graphene layers. As such, the proposed nanophotonic structure enables switchable thermal emittance that can be electrically controlled by changing the chemical potential of graphene layers. 


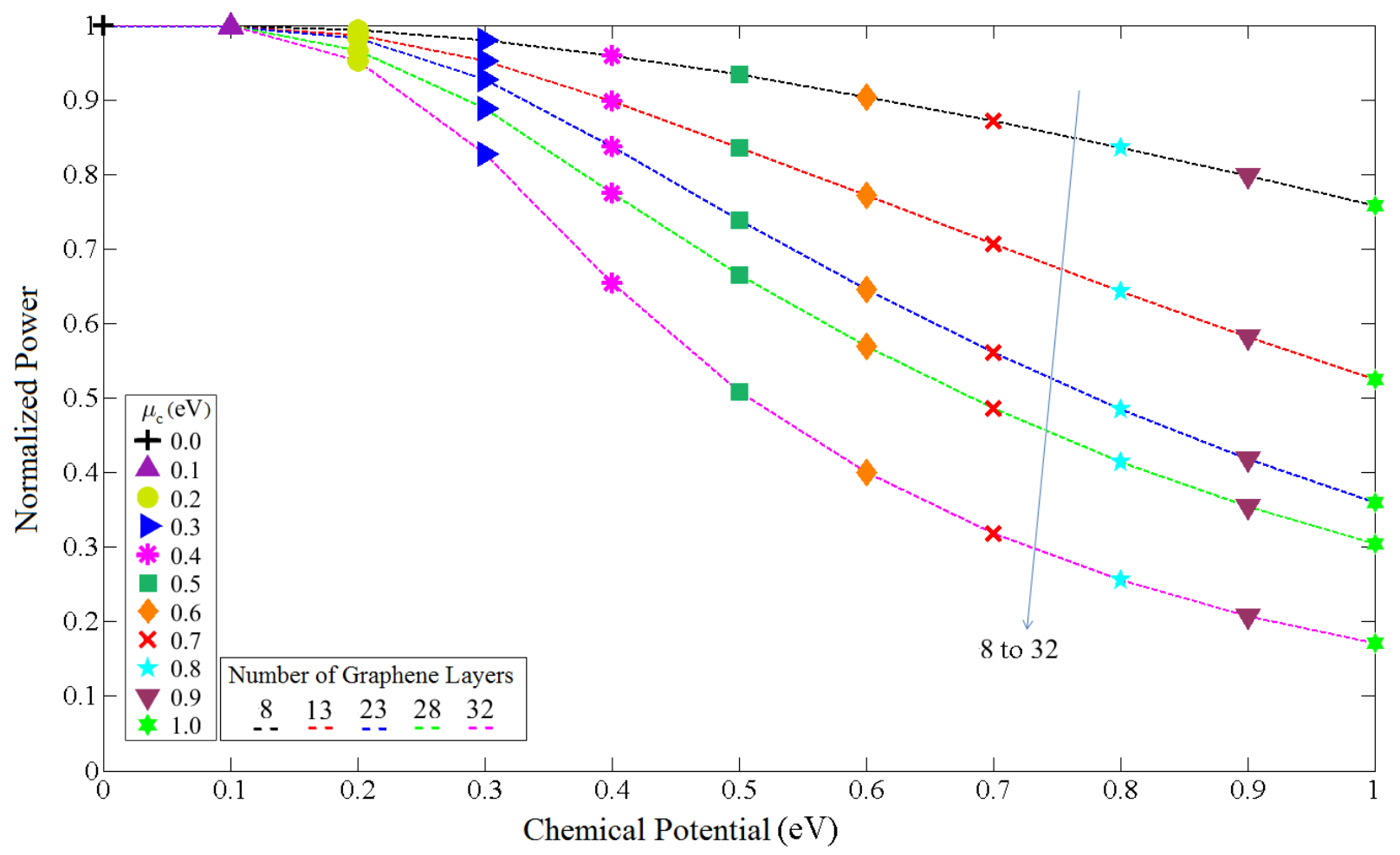

Figure 5.6: Switchability of the emitted thermal power from the optimized structures with different number of graphene layers as the chemical potential is varied. 


\section{CHAPTER 6 CONCLUDING REMARKS AND RECOMMENDED FUTURE WORK}

\subsection{Conclusion}

All materials at finite temperature emit electromagnetic radiation due to the thermally induced motion of particles and quasiparticles. Dynamic control of this radiation could enable the design of novel infrared sources. However, the spectral characteristics of the radiated thermal power are dictated by the electromagnetic energy density and emissivity, which are ordinarily fixed properties of the material and temperature.

In this thesis, we presented new aperiodic multilayer structures as selective, tunable and switchable thermal emitters. These structures composed of multiple layers of graphene and hBN are optimized by the genetic algorithm to obtain perfect absorptance/emittance in a narrow wavelength range in the infrared.

More specifically, five aperiodic thermal emitters with $8,13,23,28$, and 32 layers of graphene are optimized at zero chemical potential $\mu_{c}=0.0 \mathrm{eV}$, i.e. zero perpendicular DC electric field. The optimization took place at $\lambda=3.34 \mu \mathrm{m}$, which corresponds to the maximum power emitted from a thermal source at $\mathrm{T}=873 \mathrm{~K}$. It has been shown that the optical properties of graphene can be significantly manipulated by varying the chemical potential, leading to electrically controllable thermal emitter sources.

Despite the broadband spectra of thermal radiation, the graphene-based nanostructures enable narrowband power emission in the infrared. It has been demonstrated that the structure with larger number of graphene layers shows broader bandwidth of thermal power emission, i.e. lower selectivity. More specifically, the power emitted from the structure with 32 graphene layers is about three times broader than that of the structure with 8 graphene layers. 
The increase in the number of graphene layers, however, enhances the effects of chemical potential, promoting larger tunability and stronger switchability. The increase in the chemical potential results in a spectral shift towards shorter wavelengths, as well as decreased power emitted from the thermal source at the wavelength of $\lambda=3.34 \mu \mathrm{m}$ at which the structures are optimized. More specifically, the shift of the peak emission wavelength for the structure with 32 graphene layers is $\sim 3.5$ times larger than that of the structure with 8 graphene layers.

The increase in the number of graphene layers also enhances the switchability by changing the chemical potential. The spectral shift towards shorter wavelengths results in decreased power emitted from the thermal source at the wavelength at which the structures are optimized, i.e. $\lambda=$ $3.34 \mu \mathrm{m}$. The power emitted from the structure with 8 graphene layers decreases by $\sim 25 \%$ by increasing the chemical potential form $0.0 \mathrm{eV}$ to $1.0 \mathrm{eV}$. In addition, the range of the change in the normalized power is increased by increasing the number of graphene layers in the aperiodic multilayer structures, so that the structure with 32 graphene layers can decrease by $\sim 83 \%$ the normalized power emitted for $\mu=1.0 \mathrm{eV}$, which is $\sim 4.5$ times stronger decrease than for the structure with 8 graphene layers. As such, these structures enable a switchable thermal emitter at a given wavelength that can be electrically controlled by changing the chemical potential of graphene layers.

In summary, the dynamic control of the proposed graphene-based aperiodic multilayer structures could pave the way to a new class of tunable and switchable thermal sources in the infrared range of electromagnetic spectrum.

\subsection{Recommendations for future work}

A large range of different properties are observed in 2D layered materials. As such, combining them in sandwich structures (made up of two, three, four or more different layers of 
such materials) can offer even greater scope. Hybrid multilayered structures can be constructed by combining $2 \mathrm{D}$ materials with atomic precision and individual layers of very different character. The properties and functionality of these structures can be tuned to fit an enormous range of possible applications.

Transition metal dichalcogenide (TMDC) monolayers are atomically thin semiconductors of the type $\mathrm{MX}_{2}$, with $\mathrm{M}$ a transition metal atom (Mo, W, etc.) and $\mathrm{X}$ a chalcogen atom ( $\mathrm{S}, \mathrm{Se}$, or Te). Unlike graphene, some members of the TMDC family are true semiconductors with appreciable bandgaps in the range of 1-2 eV, making them suitable for near-infrared absorption and emission. Interestingly, although these materials have an indirect gap in bulk and few-layer form, upon thinning down to monolayers they become direct-gap semiconductors with strong photoluminescence $[72,73]$. The bandgaps in TMDCs have also been shown to be tunable - over a range from semiconducting to near metallic - by external electric field. As such, these new 2D materials can be investigated to possibly substitute or be combined with graphene layers in aperiodic multilayer structures such as those studied in this thesis, and potentially lead to highly tunable thermal emitters for specific applications. 


\section{REFERENCES}

[1] A. K. Geim, and K. S. Novoselov, "The rise of graphene," Nature materials, vol. 6, no. 3, pp. 183-191, 2007.

[2] F. Xia, H. Wang, D. Xiao, M. Dubey, and A. Ramasubramaniam, "Two-dimensional material nanophotonics," Nature Photonics, vol. 8, no. 12, pp. 899-907, 2014.

[3] A. Dato, Z. Lee, K.-J. Jeon, R. Erni, V. Radmilovic, T. J. Richardson, and M. Frenklach, "Clean and highly ordered graphene synthesized in the gas phase," Chemical Communications, no. 40, pp. 6095-6097, 2009.

[4] A. C. Neto, F. Guinea, N. M. Peres, K. S. Novoselov, and A. K. Geim, "The electronic properties of graphene," Reviews of modern physics, vol. 81, no. 1, pp. 109, 2009.

[5] H. Zhou, W. J. Yu, L. Liu, R. Cheng, Y. Chen, X. Huang, Y. Liu, Y. Wang, Y. Huang, and $\mathrm{X}$. Duan, "Chemical vapour deposition growth of large single crystals of monolayer and bilayer graphene," Nature communications, vol. 4, no. 2096, 2013.

[6] R. R. Nair, P. Blake, A. N. Grigorenko, K. S. Novoselov, T. J. Booth, T. Stauber, N. M. Peres, and A. K. Geim, "Fine structure constant defines visual transparency of graphene," Science, vol. 320, no. 5881, pp. 1308-1308, 2008.

[7] K. S. Novoselov, V. Fal, L. Colombo, P. Gellert, M. Schwab, and K. Kim, "A roadmap for graphene," Nature, vol. 490, no. 7419, pp. 192-200, 2012.

[8] X. Liu, T. Tyler, T. Starr, A. F. Starr, N. M. Jokerst, and W. J. Padilla, "Taming the blackbody with infrared metamaterials as selective thermal emitters," Physical review letters, vol. 107, no. 4, pp. 045901, 2011.

[9] J.-J. Greffet, R. Carminati, K. Joulain, J.-P. Mulet, S. Mainguy, and Y. Chen, "Coherent emission of light by thermal sources," Nature, vol. 416, no. 6876, pp. 61-64, 2002.

[10] S. Han, and D. Norris, "Beaming thermal emission from hot metallic bull's eyes," Optics express, vol. 18, no. 5, pp. 4829-4837, 2010.

[11] C. M. Cornelius, and J. P. Dowling, "Modification of Planck blackbody radiation by photonic band-gap structures," Physical Review A, vol. 59, no. 6, pp. 4736, 1999.

[12] S.-Y. Lin, J. Moreno, and J. Fleming, "Three-dimensional photonic-crystal emitter for thermal photovoltaic power generation," Applied Physics Letters, vol. 83, no. 2, pp. 380382, 2003.

[13] J. A. Schuller, T. Taubner, and M. L. Brongersma, "Optical antenna thermal emitters," Nature Photonics, vol. 3, no. 11, pp. 658-661, 2009.

[14] S. Maruyama, T. Kashiwa, H. Yugami, and M. Esashi, "Thermal radiation from twodimensionally confined modes in microcavities," Applied Physics Letters, vol. 79, no. 9, pp. 1393-1395, 2001.

[15] F. Kusunoki, J. Takahara, and I. Kobayashi, "Qualitative change of resonant peaks in thermal emission from periodic array of microcavities," Electronics Letters, vol. 39, no. 1, pp. 23-24, 2003. 
[16] H. Sai, Y. Kanamori, and H. Yugami, "High-temperature resistive surface grating for spectral control of thermal radiation," Applied Physics Letters, vol. 82, no. 11, pp. 1685$1687,2003$.

[17] K. Ikeda, H. Miyazaki, T. Kasaya, K. Yamamoto, Y. Inoue, K. Fujimura, T. Kanakugi, M. Okada, K. Hatade, and S. Kitagawa, "Controlled thermal emission of polarized infrared waves from arrayed plasmon nanocavities," Applied Physics Letters, vol. 92, no. 2, pp. 021117, 2008.

[18] G. Biener, N. Dahan, A. Niv, V. Kleiner, and E. Hasman, "Highly coherent thermal emission obtained by plasmonic bandgap structures," Applied Physics Letters, vol. 92, no. 8, pp. 081913, 2008.

[19] B. Liu, Y. Liu, and S. Shen, "Thermal plasmonic interconnects in graphene," Physical Review B, vol. 90, no. 19, pp. 195411, 2014.

[20] S. Thongrattanasiri, F. H. Koppens, and F. J. G. De Abajo, "Complete optical absorption in periodically patterned graphene," Physical review letters, vol. 108, no. 4, pp. 047401, 2012.

[21] N. Liu, M. Mesch, T. Weiss, M. Hentschel, and H. Giessen, "Infrared perfect absorber and its application as plasmonic sensor," Nano letters, vol. 10, no. 7, pp. 2342-2348, 2010.

[22] W. R. Chan, P. Bermel, R. C. Pilawa-Podgurski, C. H. Marton, K. F. Jensen, J. J. Senkevich, J. D. Joannopoulos, M. Soljačić, and I. Celanovic, "Toward high-energydensity, high-efficiency, and moderate-temperature chip-scale thermophotovoltaics," Proceedings of the National Academy of Sciences, vol. 110, no. 14, pp. 5309-5314, 2013.

[23] S. Basu, Y. B. Chen, and Z. Zhang, "Microscale radiation in thermophotovoltaic devicesa review," International Journal of Energy Research, vol. 31, no. 6- 7, pp. 689-716, 2007.

[24] H. Wang, and L. Wang, "Perfect selective metamaterial solar absorbers," Optics express, vol. 21, no. 106, pp. A1078-A1093, 2013.

[25] N. Moelders, M. U. Pralle, M. P. McNeal, I. Puscasu, L. Last, W. Ho, A. C. Greenwald, J. T. Daly, and E. A. Johnson, "Designing thermally uniform MEMs hot micro-bolometers," MRS Online Proceedings Library Archive, no. 729, p. U5. 2, 2002.

[26] G. Brucoli, P. Bouchon, R. Haïdar, M. Besbes, H. Benisty, and J.-J. Greffet, "High efficiency quasi-monochromatic infrared emitter," Applied Physics Letters, vol. 104, no. 8, pp. 081101, 2014.

[27] C. R. Otey, W. T. Lau, and S. Fan, "Thermal rectification through vacuum," Physical Review Letters, vol. 104, no. 15, pp. 154301, 2010.

[28] E. Rephaeli, A. Raman, and S. Fan, "Ultrabroadband photonic structures to achieve highperformance daytime radiative cooling," Nano letters, vol. 13, no. 4, pp. 1457-1461, 2013.

[29] H. Sai, H. Yugami, K. Nakamura, N. Nakagawa, H. Ohtsubo, and S. Maruyama, "Selective emission of Al2O3/Er3Al5O12 eutectic composite for thermophotovoltaic generation of electricity," Japanese Journal of Applied Physics, vol. 39, no. 4R, pp. 1957, 2000. 
[30] B. Bitnar, W. Durisch, J.-C. Mayor, H. Sigg, and H. Tschudi, "Characterisation of rare earth selective emitters for thermophotovoltaic applications," Solar Energy Materials and Solar Cells, vol. 73, no. 3, pp. 221-234, 2002.

[31] M. Laroche, R. Carminati, and J.-J. Greffet, "Coherent thermal antenna using a photonic crystal slab," Physical review letters, vol. 96, no. 12, pp. 123903, 2006.

[32] D. Smith, S. Schultz, N. Kroll, M. Sigalas, K. Ho, and C. Soukoulis, "Experimental and theoretical results for a two- dimensional metal photonic band- gap cavity," Applied Physics Letters, vol. 65, no. 5, pp. 645-647, 1994.

[33] E. Brown, and O. McMahon, "Large electromagnetic stop bands in metallodielectric photonic crystals," Applied Physics Letters, vol. 67, no. 15, pp. 2138-2140, 1995.

[34] D. L. Chan, M. Soljačić, and J. Joannopoulos, "Thermal emission and design in 2Dperiodic metallic photonic crystal slabs," Optics Express, vol. 14, no. 19, pp. 8785-8796, 2006.

[35] I. Celanovic, N. Jovanovic, and J. Kassakian, "Two-dimensional tungsten photonic crystals as selective thermal emitters," Applied Physics Letters, vol. 92, no. 19, pp. 193101, 2008.

[36] F. Reif, Fundamentals of statistical and thermal physics: Waveland Press, 2009.

[37] M. Laroche, F. Marquier, R. Carminati, and J.-J. Greffet, "Tailoring silicon radiative properties," Optics communications, vol. 250, no. 4, pp. 316-320, 2005.

[38] L. Wang, B. Lee, X. Wang, and Z. Zhang, "Spatial and temporal coherence of thermal radiation in asymmetric Fabry-Perot resonance cavities," International Journal of Heat and Mass Transfer, vol. 52, no. 13, pp. 3024-3031, 2009.

[39] L. Wang, S. Basu, and Z. Zhang, "Direct measurement of thermal emission from a FabryPerot cavity resonator," Journal of Heat Transfer, vol. 134, no. 7, pp. 072701, 2012.

[40] H. Sai, and H. Yugami, "Thermophotovoltaic generation with selective radiators based on tungsten surface gratings," Applied physics letters, vol. 85, no. 16, pp. 3399-3401, 2004.

[41] M. Pralle, N. Moelders, M. McNeal, I. Puscasu, A. Greenwald, J. Daly, E. Johnson, T. George, D. Choi, and I. El-Kady, "Photonic crystal enhanced narrow-band infrared emitters," Applied Physics Letters, vol. 81, no. 25, pp. 4685-4687, 2002.

[42] V. Rinnerbauer, Y. X. Yeng, W. R. Chan, J. J. Senkevich, J. D. Joannopoulos, M. Soljačić, and I. Celanovic, "High-temperature stability and selective thermal emission of polycrystalline tantalum photonic crystals," Optics express, vol. 21, no. 9, pp. 11482$11491,2013$.

[43] H. Miyazaki, K. Ikeda, T. Kasaya, K. Yamamoto, Y. Inoue, K. Fujimura, T. Kanakugi, M. Okada, K. Hatade, and S. Kitagawa, "Thermal emission of two-color polarized infrared waves from integrated plasmon cavities," Applied Physics Letters, vol. 92, no. 14, pp. 141114, 2008.

[44] B. Liu, W. Gong, B. Yu, P. Li, and S. Shen, "Perfect Thermal Emission by Nanoscale Transmission Line Resonators," Nano Letters, 2017.

[45] B. Liu, J. Li, and S. Shen, "Resonant Thermal Infrared Emitters in Near-and Far-fields," ACS Photonics, 2017. 
[46] I. Puscasu, and W. L. Schaich, "Narrow-band, tunable infrared emission from arrays of microstrip patches," Applied Physics Letters, vol. 92, no. 23, pp. 233102, 2008.

[47] J. Mason, S. Smith, and D. Wasserman, "Strong absorption and selective thermal emission from a midinfrared metamaterial," Applied Physics Letters, vol. 98, no. 24, pp. 241105 , 2011.

[48] M. N. Abbas, C.-W. Cheng, Y.-C. Chang, M.-H. Shih, H.-H. Chen, and S.-C. Lee, “Angle and polarization independent narrow-band thermal emitter made of metallic disk on $\mathrm{SiO}$ 2," Applied Physics Letters, vol. 98, no. 12, pp. 121116, 2011.

[49] C. Wu, B. Neuner III, J. John, A. Milder, B. Zollars, S. Savoy, and G. Shvets, "Metamaterial-based integrated plasmonic absorber/emitter for solar thermo-photovoltaic systems," Journal of Optics, vol. 14, no. 2, pp. 024005, 2012.

[50] S. Molesky, C. J. Dewalt, and Z. Jacob, "High temperature epsilon-near-zero and epsilonnear-pole metamaterial emitters for thermophotovoltaics," Optics express, vol. 21, no. 101, pp. A96-A110, 2013.

[51] C.-M. Wang, and D.-Y. Feng, "Omnidirectional thermal emitter based on plasmonic nanoantenna arrays," Optics express, vol. 22, no. 2, pp. 1313-1318, 2014.

[52] H. Miyazaki, T. Kasaya, M. Iwanaga, B. Choi, Y. Sugimoto, and K. Sakoda, "Dual-band infrared metasurface thermal emitter for CO2 sensing," Applied Physics Letters, vol. 105, no. 12, pp. 121107, 2014.

[53] J. B. Pendry, A. J. Holden, D. Robbins, and W. Stewart, "Magnetism from conductors and enhanced nonlinear phenomena," IEEE transactions on microwave theory and techniques, vol. 47, no. 11, pp. 2075-2084, 1999.

[54] S.M. Fu, Y.K.Zhong, M.H. Tu, B.R. Chen, and A. Lin, "A fully functionalized metamaterial perfect absorber with simple design and implementation," Scientific reports, vol.6, 2016.

[55] K. Aydin, V. E. Ferry, R. M. Briggs, and H. A. Atwater, "Broadband polarizationindependent resonant light absorption using ultrathin plasmonic super absorbers," Nature communications, vol. 2, pp. 517, 2011.

[56] W. Streyer, S. Law, A. Rosenberg, C. Roberts, V. Podolskiy, A. Hoffman, and D. Wasserman, "Engineering absorption and blackbody radiation in the far-infrared with surface phonon polaritons on gallium phosphide," Applied Physics Letters, vol. 104, no. 13, pp. 131105, 2014.

[57] T. Inoue, M. De Zoysa, T. Asano, and S. Noda, "Realization of narrowband thermal emission with optical nanostructures," Optica, vol. 2, no. 1, pp. 27-35, 2015.

[58] J. Cong, B. Yun, and Y. Cui, "The ratio of the kinetic inductance to the geometric inductance: a key parameter for the frequency tuning of the $\mathrm{THz}$ semiconductor split-ring resonator," Optics express, vol. 21, no. 17, pp. 20363-20375, 2013.

[59] L. Falkovsky, and A. Varlamov, "Space-time dispersion of graphene conductivity," The European Physical Journal B-Condensed Matter and Complex Systems, vol. 56, no. 4, pp. 281-284, 2007. 
[60] L. Falkovsky, and S. Pershoguba, "Optical far-infrared properties of a graphene monolayer and multilayer," Physical Review B, vol. 76, no. 15, pp. 153410, 2007.

[61] R. Alaee, M. Farhat, C. Rockstuhl, and F. Lederer, "A perfect absorber made of a graphene micro-ribbon metamaterial," Optics express, vol. 20, no. 27, pp. 28017-28024, 2012.

[62] H. Wang, Y. Yang, and L. Wang, "Infrared frequency-tunable coherent thermal sources," Journal of Optics, vol. 17, no. 4, pp. 045104, 2015.

[63] Z. Fang, Y. Wang, A. E. Schlather, Z. Liu, P. M. Ajayan, F. J. García de Abajo, P. Nordlander, X. Zhu, and N. J. Halas, "Active tunable absorption enhancement with graphene nanodisk arrays," Nano letters, vol. 14, no. 1, pp. 299-304, 2013.

[64] K. S. Novoselov, A. K. Geim, S. V. Morozov, D. Jiang, Y. Zhang, S. V. Dubonos, I. V. Grigorieva, and A. A. Firsov, "Electric field effect in atomically thin carbon films," science, vol. 306, no. 5696, pp. 666-669, 2004.

[65] N. D. Mermin, “Crystalline order in two dimensions," Physical Review, vol. 176, no. 1, pp. $250,1968$.

[66] T. N. Foundation, "The Nobel Prize in Physics 2010," http://www.nobelprize.org/nobelprizes/physics/laureates/2010/.

[67] K. F. Mak, L. Ju, F. Wang, and T. F. Heinz, "Optical spectroscopy of graphene: from the far infrared to the ultraviolet," Solid State Communications, vol. 152, no. 15, pp. 13411349, 2012.

[68] F. Xia, H. Yan, and P. Avouris, "The interaction of light and graphene: basics, devices, and applications," Proceedings of the IEEE, vol. 101, no. 7, pp. 1717-1731, 2013.

[69] G. Eda, and S. A. Maier, "Two-dimensional crystals: managing light for optoelectronics," Acs Nano, vol. 7, no. 7, pp. 5660-5665, 2013.

[70] N. Alem, R. Erni, C. Kisielowski, M. D. Rossell, W. Gannett, and A. Zettl, “Atomically thin hexagonal boron nitride probed by ultrahigh-resolution transmission electron microscopy," Physical Review B, vol. 80, no. 15, pp. 155425, 2009.

[71] C. R. Dean, A. F. Young, I. Meric, C. Lee, L. Wang, S. Sorgenfrei, K. Watanabe, T. Taniguchi, P. Kim, and K. L. Shepard, "Boron nitride substrates for high-quality graphene electronics," Nature nanotechnology, vol. 5, no. 10, pp. 722-726, 2010.

[72] Z. Liu, L. Song, S. Zhao, J. Huang, L. Ma, J. Zhang, J. Lou, and P. M. Ajayan, "Direct growth of graphene/hexagonal boron nitride stacked layers," Nano letters, vol. 11, no. 5, pp. 2032-2037, 2011.

[73] K. F. Mak, C. Lee, J. Hone, J. Shan, and T. F. Heinz, "Atomically thin MoS 2: a new directgap semiconductor,” Physical Review Letters, vol. 105, no. 13, pp. 136805, 2010.

[74] A. Splendiani, L. Sun, Y. Zhang, T. Li, J. Kim, C.-Y. Chim, G. Galli, and F. Wang, "Emerging photoluminescence in monolayer MoS2," Nano letters, vol. 10, no. 4, pp. 1271$1275,2010$.

[75] F. Xia, H. Wang, and Y. Jia, "Rediscovering black phosphorus as an anisotropic layered material for optoelectronics and electronics," Nature communications, vol. 5, 2014. 
[76] M. Buscema, D. J. Groenendijk, S. I. Blanter, G. A. Steele, H. S. van der Zant, and A. Castellanos-Gomez, "Fast and broadband photoresponse of few-layer black phosphorus field-effect transistors," Nano letters, vol. 14, no. 6, pp. 3347-3352, 2014.

[77] T. Low, A. Rodin, A. Carvalho, Y. Jiang, H. Wang, F. Xia, and A. C. Neto, "Tunable optical properties of multilayer black phosphorus thin films," Physical Review B, vol. 90, no. 7, pp. 075434, 2014.

[78] Y. M. Banadaki, and A. Srivastava, "Investigation of the width-dependent static characteristics of graphene nanoribbon field effect transistors using non-parabolic quantum-based model," Solid-State Electronics, vol. 111, pp. 80-90, 2015.

[79] K. S. Novoselov, A. K. Geim, S. Morozov, D. Jiang, M. Katsnelson, I. Grigorieva, S. Dubonos, and A. Firsov, "Two-dimensional gas of massless Dirac fermions in graphene," nature, vol. 438, no. 7065, pp. 197-200, 2005.

[80] Y. Zhang, Y.-W. Tan, H. L. Stormer, and P. Kim, "Experimental observation of the quantum Hall effect and Berry's phase in graphene," Nature, vol. 438, no. 7065, pp. 201204, 2005.

[81] Y.-W. Tan, Y. Zhang, H. L. Stormer, and P. Kim, "Temperature dependent electron transport in graphene," The European Physical Journal-Special Topics, vol. 148, no. 1, pp. 15-18, 2007.

[82] J. Xue, J. Sanchez-Yamagishi, D. Bulmash, P. Jacquod, A. Deshpande, K. Watanabe, T. Taniguchi, P. Jarillo-Herrero, and B. J. LeRoy, "Scanning tunnelling microscopy and spectroscopy of ultra-flat graphene on hexagonal boron nitride," Nature materials, vol. 10, no. 4, pp. 282-285, 2011.

[83] L. Wang, I. Meric, P. Huang, Q. Gao, Y. Gao, H. Tran, T. Taniguchi, K. Watanabe, L. Campos, and D. Muller, "One-dimensional electrical contact to a two-dimensional material," Science, vol. 342, no. 6158, pp. 614-617, 2013.

[84] J.-H. Chen, C. Jang, S. Xiao, M. Ishigami, and M. S. Fuhrer, "Intrinsic and extrinsic performance limits of graphene devices on SiO2," Nature nanotechnology, vol. 3, no. 4, pp. 206-209, 2008.

[85] A. Kuzmenko, E. Van Heumen, F. Carbone, and D. Van Der Marel, "Universal optical conductance of graphite," Physical review letters, vol. 100, no. 11, pp. 117401, 2008.

[86] A. Kumar, and C. Zhou, "The race to replace tin-doped indium oxide: which material will win?," ACS nano, vol. 4, no. 1, pp. 11-14, 2010.

[87] K. Ellmer, "Past achievements and future challenges in the development of optically transparent electrodes," Nature Photonics, vol. 6, no. 12, pp. 809-817, 2012.

[88] T.-H. Han, Y. Lee, M.-R. Choi, S.-H. Woo, S.-H. Bae, B. H. Hong, J.-H. Ahn, and T.-W. Lee, "Extremely efficient flexible organic light-emitting diodes with modified graphene anode," Nature Photonics, vol. 6, no. 2, pp. 105-110, 2012.

[89] S. Bae, S. J. Kim, D. Shin, J.-H. Ahn, and B. H. Hong, "Towards industrial applications of graphene electrodes," Physica Scripta, vol. 2012, no. T146, pp. 014024, 2012. 
[90] H. Yan, F. Xia, W. Zhu, M. Freitag, C. Dimitrakopoulos, A. A. Bol, G. Tulevski, and P. Avouris, "Infrared spectroscopy of wafer-scale graphene," Acs Nano, vol. 5, no. 12, pp. 9854-9860, 2011.

[91] V. B. Bregar, "Advantages of ferromagnetic nanoparticle composites in microwave absorbers," IEEE Transactions on Magnetics, vol. 40, no. 3, pp. 1679-1684, 2004.

[92] Z. Han, D. Li, H. Wang, X. Liu, J. Li, D. Geng, and Z. Zhang, "Broadband electromagneticwave absorption by $\mathrm{FeCo} / \mathrm{C}$ nanocapsules," Applied physics letters, vol. 95, no. 2, pp. 023114, 2009.

[93] T. Ishibashi, T. Furuta, H. Fushimi, S. Kodama, T. NAGATSUMA, N. SHIMIZU, and Y. MIYAMOTO, "InP/InGaAs uni-traveling-carrier photodiodes," IEICE transactions on electronics, vol. 83, no. 6, pp. 938-949, 2000.

[94] F. Xia, T. Mueller, Y.-m. Lin, A. Valdes-Garcia, and P. Avouris, "Ultrafast graphene photodetector," Nature nanotechnology, vol. 4, no. 12, pp. 839-843, 2009.

[95] T. Mueller, F. Xia, and P. Avouris, "Graphene photodetectors for high-speed optical communications," Nature Photonics, vol. 4, no. 5, pp. 297-301, 2010.

[96] T. Echtermeyer, L. Britnell, P. Jasnos, A. Lombardo, R. Gorbachev, A. Grigorenko, A. Geim, A. Ferrari, and K. Novoselov, "Strong plasmonic enhancement of photovoltage in graphene," arXiv preprint arXiv:1107.4176, 2011.

[97] K. Kim, J.-Y. Choi, T. Kim, S.-H. Cho, and H.-J. Chung, "A role for graphene in siliconbased semiconductor devices," Nature, vol. 479, no. 7373, pp. 338-344, 2011.

[98] M. Engel, M. Steiner, A. Lombardo, A. C. Ferrari, H. v. Löhneysen, P. Avouris, and R. Krupke, "Light-matter interaction in a microcavity-controlled graphene transistor," Nature Communications, vol. 3, pp. 906, 2012.

[99] M. Furchi, A. Urich, A. Pospischil, G. Lilley, K. Unterrainer, H. Detz, P. Klang, A. M. Andrews, W. Schrenk, and G. Strasser, "Microcavity-integrated graphene photodetector," Nano letters, vol. 12, no. 6, pp. 2773-2777, 2012.

[100] G. T. Reed, G. Mashanovich, F. Gardes, and D. Thomson, "Silicon optical modulators," Nature photonics, vol. 4, no. 8, pp. 518-526, 2010.

[101] Y. Tang, H.-W. Chen, S. Jain, J. D. Peters, U. Westergren, and J. E. Bowers, "50 Gb/s hybrid silicon traveling-wave electroabsorption modulator," Optics express, vol. 19, no. 7, pp. 5811-5816, 2011.

[102] F. Wang, Y. Zhang, C. Tian, C. Girit, A. Zettl, M. Crommie, and Y. R. Shen, "Gatevariable optical transitions in graphene," science, vol. 320, no. 5873, pp. 206-209, 2008.

[103] M. Liu, X. Yin, E. Ulin-Avila, B. Geng, T. Zentgraf, L. Ju, F. Wang, and X. Zhang, “A graphene-based broadband optical modulator," Nature, vol. 474, no. 7349, pp. 64-67, 2011.

[104] X. Gan, R.-J. Shiue, Y. Gao, K. F. Mak, X. Yao, L. Li, A. Szep, D. Walker Jr, J. Hone, and T. F. Heinz, "High-contrast electrooptic modulation of a photonic crystal nanocavity by electrical gating of graphene," Nano letters, vol. 13, no. 2, pp. 691-696, 2013. 
[105] B. Sensale-Rodriguez, T. Fang, R. Yan, M. M. Kelly, D. Jena, L. Liu, and H. Xing, "Unique prospects for graphene-based terahertz modulators," Applied Physics Letters, vol. 99, no. 11, pp. 113104, 2011.

[106] W. Tan, C. Su, R. Knize, G. Xie, L. Li, and D. Tang, "Mode locking of ceramic Nd: yttrium aluminum garnet with graphene as a saturable absorber," Applied Physics Letters, vol. 96, no. 3, pp. 031106, 2010.

[107] U. Keller, K. J. Weingarten, F. X. Kartner, D. Kopf, B. Braun, I. D. Jung, R. Fluck, C. Honninger, N. Matuschek, and J. A. Der Au, "Semiconductor saturable absorber mirrors (SESAM's) for femtosecond to nanosecond pulse generation in solid-state lasers," IEEE Journal of selected topics in quantum electronics, vol. 2, no. 3, pp. 435-453, 1996.

[108] Q. Bao, H. Zhang, Y. Wang, Z. Ni, Y. Yan, Z. X. Shen, K. P. Loh, and D. Y. Tang, "Atomic- layer graphene as a saturable absorber for ultrafast pulsed lasers," Advanced Functional Materials, vol. 19, no. 19, pp. 3077-3083, 2009.

[109] Z. Sun, T. Hasan, F. Torrisi, D. Popa, G. Privitera, F. Wang, F. Bonaccorso, D. M. Basko, and A. C. Ferrari, "Graphene mode-locked ultrafast laser," arXiv preprint arXiv:0909.0457, 2009.

[110] H. Zhang, D. Tang, R. Knize, L. Zhao, Q. Bao, and K. P. Loh, "Graphene mode locked, wavelength-tunable, dissipative soliton fiber laser," Applied Physics Letters, vol. 96, no. 11, pp. 111112, 2010.

[111] J.-L. Xu, X.-L. Li, J.-L. He, X.-P. Hao, Y.-Z. Wu, Y. Yang, and K.-J. Yang, "Performance of large-area few-layer graphene saturable absorber in femtosecond bulk laser," Applied Physics Letters, vol. 99, no. 26, pp. 261107, 2011.

[112] E. De Souza, M. Nuss, W. Knox, and D. Miller, "Wavelength-division multiplexing with femtosecond pulses," Optics letters, vol. 20, no. 10, pp. 1166-1168, 1995.

[113] W. Drexler, "Ultrahigh-resolution optical coherence tomography," Journal of biomedical optics, vol. 9, no. 1, pp. 47-74, 2004.

[114] X. Liu, D. Du, and G. Mourou, "Laser ablation and micromachining with ultrashort laser pulses," IEEE journal of quantum electronics, vol. 33, no. 10, pp. 1706-1716, 1997.

[115] F. Rana, "Graphene terahertz plasmon oscillators," IEEE Transactions on Nanotechnology, vol. 7, no. 1, pp. 91-99, 2008.

[116] Q. Bao, H. Zhang, B. Wang, Z. Ni, C. H. Y. X. Lim, Y. Wang, D. Y. Tang, and K. P. Loh, "Broadband graphene polarizer," Nature photonics, vol. 5, no. 7, pp. 411-415, 2011.

[117] A. Taflove, and S. C. Hagness, Computational electrodynamics: Artech house, 2005.

[118] R. Azzam, N. Bashara, and D. T. Burns, "Ellipsometry and polarized light: North Holland, Amsterdam, 1987 (ISBN 0-444-87016-4). xvii+ 539 pp. Price Dfl. 75.00,” Analytica Chimica Acta, vol. 199, pp. 283-284, 1987.

[119] Z. Knittl, Optics of thin films: an optical multilayer theory: Wiley London:, 1976.

[120] L. A. Pettersson, L. S. Roman, and O. Inganäs, "Modeling photocurrent action spectra of photovoltaic devices based on organic thin films," Journal of Applied Physics, vol. 86, no. 1, pp. 487-496, 1999. 
[121] Y. Rahmat-Samii, and E. Michielssen, "Electromagnetic optimization by genetic algorithms," Microwave Journal, vol. 42, no. 11, pp. 232-232, 1999.

[122] C. H. Granier, F. O. Afzal, C. Min, J. P. Dowling, and G. Veronis, "Optimized aperiodic highly directional narrowband infrared emitters," JOSA B, vol. 31, no. 6, pp. 1316-1321, 2014.

[123] C. H. Granier, F. O. Afzal, S. G. Lorenzo, M. Reyes Jr, J. P. Dowling, and G. Veronis, "Optimized aperiodic multilayer structures for use as narrow-angular absorbers," Journal of Applied Physics, vol. 116, no. 24, pp. 243101, 2014.

[124] K. Krishnakumar, "Micro-genetic algorithms for stationary and non-stationary function optimization," Symposium on Visual Communications, Image Processing, and Intelligent Robotics Systems. International Society for Optics and Photonics, vol. 1196, pp. 289-296, 1990.

[125] K. Deb, and S. Agrawal, "Understanding Interactions among Genetic Algorithm Parameters," FOGA, pp. 265-286, 1998.

[126] D. E. Goldberg, K. Deb, and J. H. Clark, "Genetic algorithms, noise, and the sizing of populations," Urbana, vol. 51, pp. 61801, 1991.

[127] R. L. Haupt, and D. H. Werner, Genetic algorithms in electromagnetics: John Wiley \& Sons, 2007.

[128] T. Stauber, N. Peres, and A. Geim, "Optical conductivity of graphene in the visible region of the spectrum," Physical Review B, vol. 78, no. 8, pp. 085432, 2008.

[129] T. Stauber, D. Noriega-Pérez, and J. Schliemann, "Universal absorption of twodimensional systems," Physical Review B, vol. 91, no. 11, pp. 115407, 2015.

[130] I. V. Iorsh, I. S. Mukhin, I. V. Shadrivov, P. A. Belov, and Y. S. Kivshar, "Hyperbolic metamaterials based on multilayer graphene structures," Physical Review B, vol. 87, no. 7, pp. 075416, 2013.

[131] V. Gusynin, and S. Sharapov, "Transport of Dirac quasiparticles in graphene: Hall and optical conductivities," Physical Review B, vol. 73, no. 24, pp. 245411, 2006.

[132] V. Gusynin, S. Sharapov, and J. Carbotte, "Unusual microwave response of Dirac quasiparticles in graphene," Physical review letters, vol. 96, no. 25, pp. 256802, 2006.

[133] V. Gusynin, S. Sharapov, and J. Carbotte, "Magneto-optical conductivity in graphene," Journal of Physics: Condensed Matter, vol. 19, no. 2, pp. 026222, 2006.

[134] A. Vakil, and N. Engheta, "Transformation optics using graphene," Science, vol. 332, no. 6035, pp. 1291-1294, 2011.

[135] T. Ando, Y. Zheng, and H. Suzuura, "Dynamical conductivity and zero-mode anomaly in honeycomb lattices," Journal of the Physical Society of Japan, vol. 71, no. 5, pp. 13181324, 2002.

[136] B. Wunsch, T. Stauber, F. Sols, and F. Guinea, "Dynamical polarization of graphene at finite doping," New Journal of Physics, vol. 8, no. 12, pp. 318, 2006. 
[137] G. W. Hanson, “Dyadic Green's functions for an anisotropic, non-local model of biased graphene," IEEE Transactions on Antennas and Propagation, vol. 56, no. 3, pp. 747-757, 2008.

[138] T. Wehling, K. Novoselov, S. Morozov, E. Vdovin, M. Katsnelson, A. Geim, and A. Lichtenstein, "Molecular doping of graphene," Nano letters, vol. 8, no. 1, pp. 173-177, 2008.

[139] R. A. Depine, "A graphene optics: Electromagnetic solution of canonical problems." Morgan \& Claypool Publishers, 2017.

[140] A. Vakil, "Transformation optics using graphene: One-atom-thick optical devices based on graphene," University of Pennsylvania, 2012.

[141] G. W. Hanson, "Dyadic Green's functions and guided surface waves for a surface conductivity model of graphene," Journal of Applied Physics, vol. 103, no. 6, pp. 064302, 2008.

[142] V. Gusynin, S. Sharapov, and J. Carbotte, "Sum rules for the optical and Hall conductivity in graphene," Physical Review B, vol. 75, no. 16, pp. 165407, 2007.

[143] J. M. Dawlaty, S. Shivaraman, J. Strait, P. George, M. Chandrashekhar, F. Rana, M. G. Spencer, D. Veksler, and Y. Chen, "Measurement of the optical absorption spectra of epitaxial graphene from terahertz to visible," Applied Physics Letters, vol. 93, no. 13, pp. 131905, 2008.

[144] B. Sensale-Rodriguez, R. Yan, M. M. Kelly, T. Fang, K. Tahy, W. S. Hwang, D. Jena, L. Liu, and H. G. Xing, "Broadband graphene terahertz modulators enabled by intraband transitions," Nature communications, vol. 3, pp. 780, 2012.

[145] A. Andryieuski, and A. V. Lavrinenko, "Graphene metamaterials based tunable terahertz absorber: effective surface conductivity approach," Optics express, vol. 21, no. 7, pp. 91449155, 2013.

[146] M. Jablan, H. Buljan, and M. Soljačić, "Plasmonics in graphene at infrared frequencies," Physical review B, vol. 80, no. 24, pp. 245435, 2009.

[147] S. H. Mousavi, I. Kholmanov, K. B. Alici, D. Purtseladze, N. Arju, K. Tatar, D. Y. Fozdar, J. W. Suk, Y. Hao, and A. B. Khanikaev, "Inductive tuning of Fano-resonant metasurfaces using plasmonic response of graphene in the mid-infrared," Nano letters, vol. 13, no. 3, pp. 1111-1117, 2013.

[148] Y. Zhang, Y. Feng, B. Zhu, J. Zhao, and T. Jiang, "Graphene based tunable metamaterial absorber and polarization modulation in terahertz frequency," Optics express, vol. 22, no. 19, pp. 22743-22752, 2014.

[149] I.-T. Lin, Y.-P. Lai, K.-H. Wu, and J.-M. Liu, "Terahertz Optoelectronic Property of Graphene: Substrate-Induced Effects on Plasmonic Characteristics," Applied Sciences, vol. 4, no. 1, pp. 28-41, 2014.

[150] M. Ghebrebrhan, P. Bermel, Y. Yeng, I. Celanovic, M. Soljačić, and J. Joannopoulos, "Tailoring thermal emission via $\mathrm{Q}$ matching of photonic crystal resonances," Physical Review A, vol. 83, no. 3, pp. 033810, 2011. 
[151] E. Rephaeli, and S. Fan, "Absorber and emitter for solar thermo-photovoltaic systems to achieve efficiency exceeding the Shockley-Queisser limit," Optics express, vol. 17, no. 17, pp. 15145-15159, 2009.

[152] F. Marquier, J.-J. Greffet, S. Collin, F. Pardo, and J. Pelouard, "Resonant transmission through a metallic film due to coupled modes," Optics express, vol. 13, no. 1, pp. 70-76, 2005.

[153] J. Fleming, S. Lin, I. El-Kady, R. Biswas, and K. Ho, "All-metallic three-dimensional photonic crystals with a large infrared bandgap," Nature, vol. 417, no. 6884, pp. 52-55, 2002.

[154] M. Florescu, H. Lee, I. Puscasu, M. Pralle, L. Florescu, D. Z. Ting, and J. P. Dowling, "Improving solar cell efficiency using photonic band-gap materials," Solar Energy Materials and Solar Cells, vol. 91, no. 17, pp. 1599-1610, 2007.

[155] H. Yan, X. Li, B. Chandra, G. Tulevski, Y. Wu, M. Freitag, W. Zhu, P. Avouris, and F. $\mathrm{Xia}$, "Tunable infrared plasmonic devices using graphene/insulator stacks," Nature nanotechnology, vol. 7, no. 5, pp. 330-334, 2012.

[156] N. Mattiucci, G. D’Aguanno, A. Alu, C. Argyropoulos, J. Foreman, and M. Bloemer, "Taming the thermal emissivity of metals: A metamaterial approach," Applied Physics Letters, vol. 100, no. 20, pp. 201109, 2012.

[157] S. Singh, J. Potopowicz, L. Van Uitert, and S. Wemple, "Nonlinear optical properties of hexagonal silicon carbide," Applied Physics Letters, vol. 19, no. 3, pp. 53-56, 1971.

[158] M. Fernández-Perea, J. I. Larruquert, J. A. Aznárez, J. A. Méndez, M. Vidal-Dasilva, E. Gullikson, A. Aquila, R. Soufli, and J. Fierro, "Optical constants of electron-beam evaporated boron films in the 6.8-900 eV photon energy range," JOSA A, vol. 24, no. 12, pp. 3800-3807, 2007.

[159] L. Aksyutov, "Temperature dependence of the optical constants of tungsten and gold," Journal of Applied Spectroscopy, vol. 26, no. 5, pp. 656-660, 1977.

[160] Y. X. Yeng, M. Ghebrebrhan, P. Bermel, W. R. Chan, J. D. Joannopoulos, M. Soljačić, and I. Celanovic, "Enabling high-temperature nanophotonics for energy applications," Proceedings of the National Academy of Sciences, vol. 109, no. 7, pp. 2280-2285, 2012.

[161] Y. X. Yeng, J. B. Chou, V. Rinnerbauer, Y. Shen, S.-G. Kim, J. D. Joannopoulos, M. Soljacic, and I. Čelanović, "Global optimization of omnidirectional wavelength selective emitters/absorbers based on dielectric-filled anti-reflection coated two-dimensional metallic photonic crystals," Optics express, vol. 22, no. 18, pp. 21711-21718, 2014.

[162] G. Veronis, R. W. Dutton, and S. Fan, "Metallic photonic crystals with strong broadband absorption at optical frequencies over wide angular range," Journal of applied physics, vol. 97, no. 9, pp. 093104, 2005.

[163] G. Devarapu, and S. Foteinopoulou, "Mid-IR near-perfect absorption with a SiC photonic crystal with angle-controlled polarization selectivity," Optics express, vol. 20, no. 12, pp. 13040-13054, 2012. 
[164] T. Søndergaard, and S. I. Bozhevolnyi, "Theoretical analysis of plasmonic black gold: periodic arrays of ultra-sharp grooves," New Journal of Physics, vol. 15, no. 1, pp. 013034 , 2013.

[165] O. Stenzel, A. Stendal, K. Voigtsberger, and C. Von Borczyskowski, "Enhancement of the photovoltaic conversion efficiency of copper phthalocyanine thin film devices by incorporation of metal clusters," Solar energy materials and solar cells, vol. 37, no. 3-4, pp. 337-348, 1995.

[166] M. Westphalen, U. Kreibig, J. Rostalski, H. Lüth, and D. Meissner, "Metal cluster enhanced organic solar cells," Solar Energy Materials and Solar Cells, vol. 61, no. 1, pp. 97-105, 2000.

[167] S. Lin, J. Fleming, Z. Li, I. El-Kady, R. Biswas, and K. M. Ho, "Origin of absorption enhancement in a tungsten, three-dimensional photonic crystal," JOSA B, vol. 20, no. 7, pp. 1538-1541, 2003.

[168] A. Sharma, S. H. Zaidi, P. Logofatu, and S. Brueck, "Optical and electrical properties of nanostructured metal-silicon-metal photodetectors," IEEE journal of quantum electronics, vol. 38, no. 12, pp. 1651-1660, 2002.

[169] H. Huang, Y. Huang, X. Wang, Q. Wang, and X. Ren, "Long wavelength resonant cavity photodetector based on InP/air-gap Bragg reflectors," IEEE Photonics Technology Letters, vol. 16, no. 1, pp. 245-247, 2004.

[170] A. Tittl, P. Mai, R. Taubert, D. Dregely, N. Liu, and H. Giessen, "Palladium-based plasmonic perfect absorber in the visible wavelength range and its application to hydrogen sensing," Nano letters, vol. 11, no. 10, pp. 4366-4369, 2011.

[171] J. Hendrickson, J. Guo, B. Zhang, W. Buchwald, and R. Soref, "Wideband perfect light absorber at midwave infrared using multiplexed metal structures," Optics letters, vol. 37, no. 3, pp. 371-373, 2012.

[172] H. Chenggang, Z. Zeyu, C. Xunan, and L. Xiangang, "Realizing near-perfect absorption at visible frequencies," Optics express, vol.17, no.13, pp.11039-11044, 2009.

[173] M. K. Hedayati, M. Javaherirahim, B. Mozooni, R. Abdelaziz, A. Tavassolizadeh, V. S. K. Chakravadhanula, V. Zaporojtchenko, T. Strunkus, F. Faupel, and M. Elbahri, "Design of a perfect black absorber at visible frequencies using plasmonic metamaterials," Advanced Materials, vol. 23, no. 45, pp. 5410-5414, 2011.

[174] G. Devarapu, and S. Foteinopoulou, "Broadband Mid-IR superabsorption with aperiodic polaritonic photonic crystals," Journal of the European Optical Society-Rapid publications, vol. 9, 2014.

[175] R. Zhang, and Z. Zhang, "Tunable positive and negative refraction of infrared radiation in graphene-dielectric multilayers," Applied Physics Letters, vol. 107, no. 19, pp. 191112, 2015. 


\section{APPENDIX A \\ DATA FOR THE THICKNESSES OF OPTIMIZED STRUCTURES}

\begin{tabular}{|c|c|c|c|c|}
\hline $\begin{array}{l}\text { Optimized Structure } \\
\text { with } 8 \text { Layers of } \\
\text { Graphene }\end{array}$ & $\begin{array}{c}\text { Optimized Structure } \\
\text { with } 13 \text { Layers of } \\
\text { Graphene }\end{array}$ & $\begin{array}{l}\text { Optimized Structure } \\
\text { with } 23 \text { Layers of } \\
\text { Graphene }\end{array}$ & $\begin{array}{l}\text { Optimized Structure } \\
\text { with } 28 \text { Layers of } \\
\text { Graphene }\end{array}$ & $\begin{array}{c}\text { Optimized Structure } \\
\text { with } 32 \text { Layers of } \\
\text { Graphene }\end{array}$ \\
\hline $\begin{array}{l}\text { SiC }(284.9 \mathrm{~nm}) \\
\text { Graphene } \\
\text { hBN }(65.66 \mathrm{~nm}) \\
\text { Graphene } \\
\text { hBN }(66.33 \mathrm{~nm}) \\
\text { Graphene } \\
\text { hBN }(65.34 \mathrm{~nm}) \\
\text { Graphene } \\
\text { hBN }(51.81 \mathrm{~nm}) \\
\text { Graphene } \\
\text { hBN }(64.35 \mathrm{~nm}) \\
\text { Graphene } \\
\text { hBN }(18.15 \mathrm{~nm}) \\
\text { Graphene } \\
\text { hBN }(36.96 \mathrm{~nm}) \\
\text { Graphene } \\
\text { SiC }(244.6 \mathrm{~nm}) \\
\text { Tungsten }\end{array}$ & $\begin{array}{l}\text { SiC }(372.9 \mathrm{~nm}) \\
\text { Graphene } \\
\text { hBN }(45.87 \mathrm{~nm}) \\
\text { Graphene } \\
\text { hBN }(4.95 \mathrm{~nm}) \\
\text { Graphene } \\
\text { hBN }(6.93 \mathrm{~nm}) \\
\text { Graphene } \\
\text { hBN }(9.24 \mathrm{~nm}) \\
\text { Graphene } \\
\text { hBN }(46.2 \mathrm{~nm}) \\
\text { Graphene } \\
\text { hBN }(27.06 \mathrm{~nm}) \\
\text { Graphene } \\
\text { hBN }(43.23 \mathrm{~nm}) \\
\text { Graphene } \\
\text { hBN }(21.7 \mathrm{~nm}) \\
\text { Graphene } \\
\text { hBN }(29.7 \mathrm{~nm}) \\
\text { Graphene } \\
\text { hBN }(10.89 \mathrm{~nm}) \\
\text { Graphene } \\
\text { hBN }(0.33 \mathrm{~nm}) \\
\text { Graphene } \\
\text { hBN }(7.26 \mathrm{~nm}) \\
\text { Graphene } \\
\text { SiC }(257.6 \mathrm{~nm}) \\
\text { Tungsten }\end{array}$ & $\begin{array}{l}\text { SiC (164.6 nm) } \\
\text { Graphene } \\
\text { hBN }(35.64 \mathrm{~nm}) \\
\text { Graphene } \\
\text { hBN }(35.64 \mathrm{~nm}) \\
\text { Graphene } \\
\text { hBN }(8.58 \mathrm{~nm}) \\
\text { Graphene } \\
\text { hBN }(33.6 \mathrm{~nm}) \\
\text { Graphene } \\
\text { hBN }(23.76 \mathrm{~nm}) \\
\text { Graphene } \\
\text { hBN }(36.63 \mathrm{~nm}) \\
\text { Graphene } \\
\text { hBN }(31.68 \mathrm{~nm}) \\
\text { Graphene } \\
\text { hBN }(28.38 \mathrm{~nm}) \\
\text { Graphene } \\
\text { hBN }(16.83 \mathrm{~nm}) \\
\text { Graphene } \\
\text { hBN }(13.2 \mathrm{~nm}) \\
\text { Graphene } \\
\text { hBN }(45.87 \mathrm{~nm}) \\
\text { Graphene } \\
\text { hBN }(14.85 \mathrm{~nm}) \\
\text { Graphene } \\
\text { hBN }(18.48 \mathrm{~nm}) \\
\text { Graphene } \\
\text { hBN }(31.02 \mathrm{~nm}) \\
\text { Graphene } \\
\text { hBN }(38.94 \mathrm{~nm}) \\
\text { Graphene } \\
\text { hBN }(37.29 \mathrm{~nm}) \\
\text { Graphene } \\
\text { hBN }(11.55 \mathrm{~nm}) \\
\text { Graphene } \\
\text { hBN }(33.33 \mathrm{~nm}) \\
\text { Graphene } \\
\text { hBN }(42.9 \mathrm{~nm}) \\
\text { Graphene } \\
\text { hBN }(33.33 \mathrm{~nm}) \\
\text { Graphene } \\
\text { hBN }(42.9 \mathrm{~nm}) \\
\text { Graphene } \\
\text { hBN }(34.65 \mathrm{~nm}) \\
\text { Graphene } \\
\text { SiC }(176.7 \mathrm{~nm}) \\
\text { Tungsten } \\
\end{array}$ & $\begin{array}{l}\text { SiC (142.1 nm) } \\
\text { Graphene } \\
\text { hBN }(18.48 \mathrm{~nm}) \\
\text { Graphene } \\
\text { hBN }(10.56 \mathrm{~nm}) \\
\text { Graphene } \\
\text { hBN }(26.4 \mathrm{~nm}) \\
\text { Graphene } \\
\text { hBN }(5.28 \mathrm{~nm}) \\
\text { Graphene } \\
\text { hBN }(19.47 \mathrm{~nm}) \\
\text { Graphene } \\
\text { hBN }(46.86 \mathrm{~nm}) \\
\text { Graphene } \\
\text { hBN }(1.65 \mathrm{~nm}) \\
\text { Graphene } \\
\text { hBN }(8.25 \mathrm{~nm}) \\
\text { Graphene } \\
\text { hBN }(36.96 \mathrm{~nm}) \\
\text { Graphene } \\
\text { hBN }(32.01 \mathrm{~nm}) \\
\text { Graphene } \\
\text { hBN }(1.65 \mathrm{~nm}) \\
\text { Graphene } \\
\text { hBN }(49.83 \mathrm{~nm}) \\
\text { Graphene } \\
\text { hBN }(31.3 \mathrm{~nm}) \\
\text { Graphene } \\
\text { hBN }(43.89 \mathrm{~nm}) \\
\text { Graphene } \\
\text { hBN }(12.87 \mathrm{~nm}) \\
\text { Graphene } \\
\text { hBN }(37.29 \mathrm{~nm}) \\
\text { Graphene } \\
\text { hBN }(10.56 \mathrm{~nm}) \\
\text { Graphene } \\
\text { hBN }(48.18 \mathrm{~nm}) \\
\text { Graphene } \\
\text { hBN }(27.06 \mathrm{~nm}) \\
\text { Graphene } \\
\text { hBN }(45.21 \mathrm{~nm}) \\
\text { Graphene } \\
\text { hBN }(38.61 \mathrm{~nm}) \\
\text { Graphene } \\
\text { hBN }(22.77 \mathrm{~nm}) \\
\text { Graphene } \\
\text { hBN }(36.3 \mathrm{~nm}) \\
\text { Graphene } \\
\end{array}$ & $\begin{array}{l}\text { SiC (174.6 nm) } \\
\text { Graphene } \\
\text { hBN }(11.22 \mathrm{~nm}) \\
\text { Graphene } \\
\text { hBN9 }(1.32 \mathrm{~nm}) \\
\text { Graphene } \\
\text { hBN }(17.49 \mathrm{~nm}) \\
\text { Graphene } \\
\text { hBN }(22.44 \mathrm{~nm}) \\
\text { Graphene } \\
\text { hBN }(15.18 \mathrm{~nm}) \\
\text { Graphene } \\
\text { hBN }(2.31 \mathrm{~nm}) \\
\text { Graphene } \\
\text { hBN }(5.94 \mathrm{~nm}) \\
\text { Graphene } \\
\text { hBN }(44.55 \mathrm{~nm}) \\
\text { Graphene } \\
\text { hBN }(26.07 \mathrm{~nm}) \\
\text { Graphene } \\
\text { hBN }(20.46 \mathrm{~nm}) \\
\text { Graphene } \\
\text { hBN }(32.34 \mathrm{~nm}) \\
\text { Graphene } \\
\text { hBN }(4.29 \mathrm{~nm}) \\
\text { Graphene } \\
\text { hBN }(4.58 \mathrm{~nm}) \\
\text { Graphene } \\
\text { hBN }(3.63 \mathrm{~nm}) \\
\text { Graphene } \\
\text { hBN }(45.21 \mathrm{~nm}) \\
\text { Graphene } \\
\text { hBN }(6.93 \mathrm{~nm}) \\
\text { Graphene } \\
\text { hBN }(30.36 \mathrm{~nm}) \\
\text { Graphene } \\
\text { hBN }(38.61 \mathrm{~nm}) \\
\text { Graphene } \\
\text { hBN }(33.99 \mathrm{~nm}) \\
\text { Graphene } \\
\text { hBN }(43.89 \mathrm{~nm}) \\
\text { Graphene } \\
\text { hBN }(33.99 \mathrm{~nm}) \\
\text { Graphene } \\
\text { hBN }(9.24 \mathrm{~nm}) \\
\text { Graphene } \\
\text { hBN }(11.22 \mathrm{~nm}) \\
\text { Graphene } \\
\end{array}$ \\
\hline
\end{tabular}




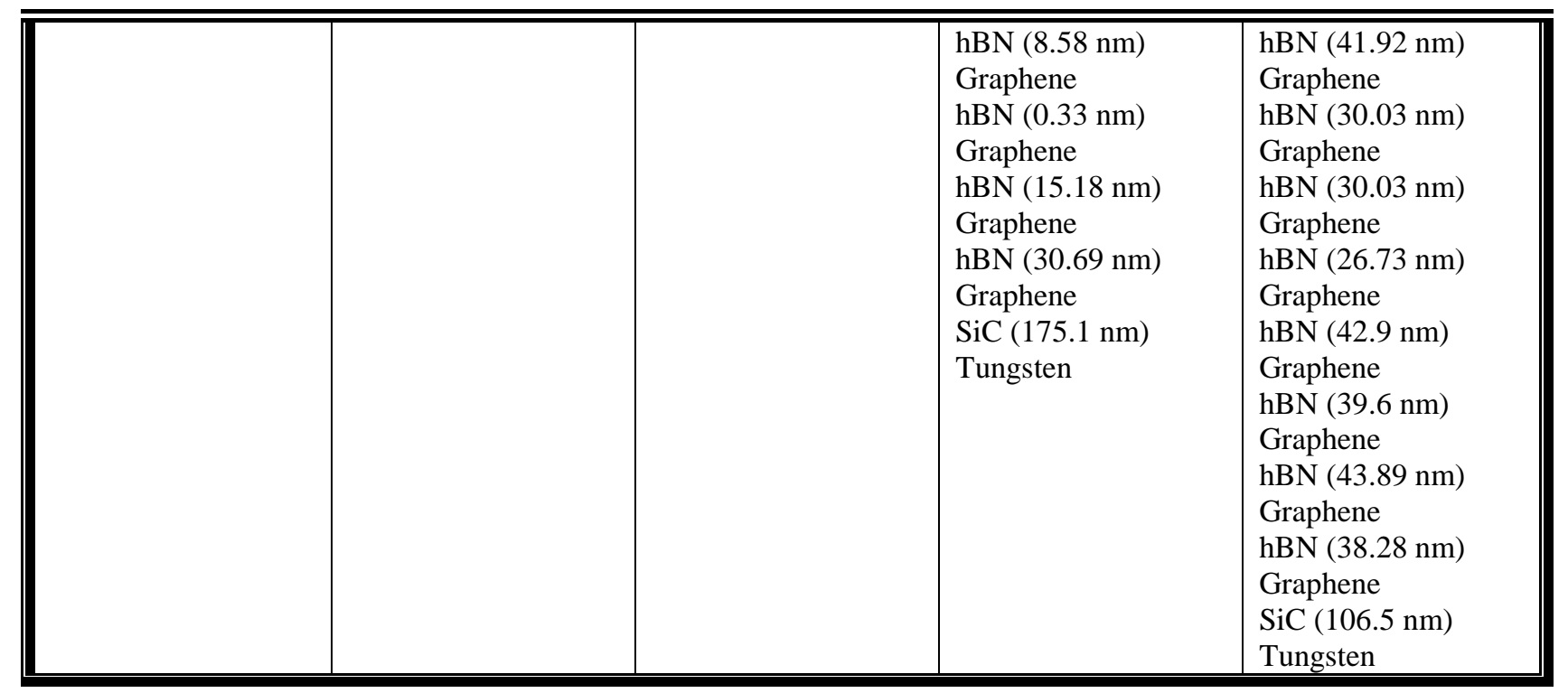




\section{APPENDIX B LETTER OF PERMISSION FOR ADOPTED FIGURES}

Figure 2.1 is adopted by permission from [1].

\begin{tabular}{|c|c|}
\hline $\begin{array}{l}\text { Licensed Content } \\
\text { Publisher }\end{array}$ & Nature Publishing Group \\
\hline $\begin{array}{l}\text { Licensed Content } \\
\text { Publication }\end{array}$ & Nature Materials \\
\hline Licensed Content Title & The rise of graphene \\
\hline Licensed Content Author & A. K. GeimandK. S. Novoselov \\
\hline Licensed Content Date & Mar 1, 2007 \\
\hline Licensed Content Volume & 6 \\
\hline Licensed Content Issue & 3 \\
\hline Type of Use & reuse in a dissertation / thesis \\
\hline Requestor type & academic/educational \\
\hline Format & electronic \\
\hline Portion & figures/tables/illustrations \\
\hline
\end{tabular}

Figure 2.2 is adopted by permission from [2].

\begin{tabular}{|c|c|}
\hline License Number & 4115870226007 \\
\hline License date & May 25, 2017 \\
\hline $\begin{array}{l}\text { Licensed Content } \\
\text { Publisher }\end{array}$ & Nature Publishing Group \\
\hline $\begin{array}{l}\text { Licensed Content } \\
\text { Publication }\end{array}$ & Nature Photonics \\
\hline Licensed Content Title & Two-dimensional material nanophotonics \\
\hline Licensed Content Author & Fengnian Xia, Han Wang, Di Xiao, Madan Dubey, Ashwin Ramasubramaniam \\
\hline Licensed Content Date & Nov 27,2014 \\
\hline Licensed Content Volume & 8 \\
\hline Licensed Content Issue & 12 \\
\hline Type of Use & reuse in a dissertation / thesis \\
\hline Requestor type & non-commercial (non-profit) \\
\hline Format & electronic \\
\hline Portion & figures/tables/illustrations \\
\hline $\begin{array}{l}\text { Number of } \\
\text { figures/tables/illustrations }\end{array}$ & 2 \\
\hline High-res required & no \\
\hline Figures & Figure 1 and 6 \\
\hline
\end{tabular}

Figure 2.3(a) is adopted by permission from [3].

\begin{tabular}{|c|c|}
\hline $\begin{array}{l}\text { Licensed Content } \\
\text { Publisher }\end{array}$ & Royal Society of Chemistry \\
\hline $\begin{array}{l}\text { Licensed Content } \\
\text { Publication }\end{array}$ & Chemical Communications (Cambridge) \\
\hline Licensed Content Title & Clean and highly ordered graphene synthesized in the gas phase \\
\hline $\begin{array}{l}\text { Licensed Content } \\
\text { Author }\end{array}$ & $\begin{array}{l}\text { Albert Dato, Zonghoon Lee, Ki-Joon Jeon, Rolf Erni, Velimir Radmilovic, Thomas J. } \\
\text { Richardson, Michael Frenklach }\end{array}$ \\
\hline Licensed Content Date & Aug 24, 2009 \\
\hline Licensed Content Issue & 40 \\
\hline Type of Use & Thesis/Dissertation \\
\hline Requestor type & non-commercial (non-profit) \\
\hline Portion & figures/tables/images \\
\hline $\begin{array}{l}\text { Number of } \\
\text { figures/tables/images }\end{array}$ & 1 \\
\hline
\end{tabular}


Figure 2.3(b), 2.3(c) and 2.4(a) are adopted by permission from [4].

\begin{tabular}{|c|c|}
\hline License Number & 4093260638765 \\
\hline License date & Apr 20, 2017 \\
\hline $\begin{array}{l}\text { Licensed Content } \\
\text { Publisher }\end{array}$ & American Physical Society \\
\hline $\begin{array}{l}\text { Licensed Content } \\
\text { Publication }\end{array}$ & Reviews of Modern Physics \\
\hline Licensed Content Title & The electronic properties of graphene \\
\hline Licensed Content Author & A. H. Castro Neto et al. \\
\hline Licensed Content Date & Jan 14,2009 \\
\hline $\begin{array}{l}\text { Licensed Content } \\
\text { Volume }\end{array}$ & 81 \\
\hline Type of use & Thesis/Dissertation \\
\hline Requestor type & Student \\
\hline Format & Electronic \\
\hline Portion & image/photo \\
\hline
\end{tabular}

Figure 2.5 is adopted by permission from [6].

AAAS reprint and permission at http://www.sciencemag.org/help/reprints-and-permissions, quoted "If you are using figure(s)/table(s), permission is granted for use in print and electronic versions of your dissertation or thesis."

Figure 2.6 is adopted by permission from [7].

\begin{tabular}{|c|c|}
\hline License Number & 4115860896924 \\
\hline License date & May 25,2017 \\
\hline $\begin{array}{l}\text { Licensed Content } \\
\text { Publisher }\end{array}$ & Nature Publishing Group \\
\hline $\begin{array}{l}\text { Licensed Content } \\
\text { Publication }\end{array}$ & Nature \\
\hline Licensed Content Title & A roadmap for graphene \\
\hline Licensed Content Author & K. S. Novoselov, V. I. Fal'ko, L. Colombo, P. R. Gellert, M. G. Schwab, K. Kim \\
\hline Licensed Content Date & Oct 10,2012 \\
\hline Licensed Content Volume & 490 \\
\hline Licensed Content Issue & 7419 \\
\hline Type of Use & reuse in a dissertation / thesis \\
\hline Requestor type & non-commercial (non-profit) \\
\hline Format & electronic \\
\hline Portion & figures/tables/illustrations \\
\hline $\begin{array}{l}\text { Number of } \\
\text { figures/tables/illustrations }\end{array}$ & 2 \\
\hline High-res required & no \\
\hline Figures & Figure 2 and 3 \\
\hline
\end{tabular}




\section{VITA}

Safura Sharifi was born in 1983 in Tehran, Iran. She graduated her B.Sc. in Electrical Engineering from Azad Tehran University in 2007. She worked at Samsung Co., Tehran for 8 years, from 2002 to 2010. She proceeded her eduation to get M.Sc. in electrical engineering from Shiraz University of Technology from 2010 to 2012. In January 2016, she begin studying at Louisiana State University to pursue graduate studies. She is currently a candidate for Master of Science in Electrical Engineering which will be awarded in August 2017. 